\title{
CONTINUITY, POSITIVITY AND SIMPLICITY OF THE LYAPUNOV EXPONENTS FOR QUASI-PERIODIC COCYCLES
}

\author{
PEDRO DUARTE AND SILVIUS KLEIN
}

\begin{abstract}
An analytic quasi-periodic cocycle is a linear cocycle over a fixed ergodic torus translation of one or several variables, where the fiber action depends analytically on the base point. Consider the space of all such cocycles of any given dimension and endow it with the uniform norm. Assume that the translation vector satisfies a generic Diophantine condition. We prove large deviation type estimates for the iterates of such cocycles, which, moreover, are stable under small perturbations of the cocycle. As a consequence of these uniform estimates, we establish continuity properties of the Lyapunov exponents regarded as functions on this space of cocycles. This result builds upon our previous work on this topic and its proof uses an abstract continuity theorem of the Lyapunov exponents which we derived in a recent monograph. The new feature of this paper is extending the availability of such results to cocycles that are identically singular (i.e. non-invertible anywhere), in the several variables torus translation setting. This feature is exactly what allows us, through a simple limiting argument, to obtain criteria for the positivity and simplicity of the Lyapunov exponents of such cocycles. Specializing to the family of cocycles corresponding to a block Jacobi operator, we derive consequences on the continuity, positivity and simplicity of its Lyapunov exponents, and on the continuity of its integrated density of states.
\end{abstract}

\section{InTRODUCTION AND STATEMENTS}

Definitions, notations, framework. In ergodic theory, a linear cocycle is a dynamical system on a vector bundle, which preserves the linear bundle structure and induces a measure preserving dynamical system on the base. The vector bundle is usually assumed to be trivial and the base dynamics to be an ergodic measure preserving transformation $T: X \rightarrow X$ on some probability space $(X, \mathcal{F}, \mu)$. Given a measurable function $A: X \rightarrow \operatorname{Mat}_{m}(\mathbb{R})$, the map $F: X \times \mathbb{R}^{m} \rightarrow X \times \mathbb{R}^{m}$ defined by $F(x, v)=(T x, A(x) v)$ is a linear cocycle over $T$. The iterated maps $F^{n}$ are given by $F^{n}(x, v)=\left(T^{n} x, A^{(n)}(x) v\right)$, where for all $x \in X$ and $n \geq 1, A^{(n)}(x):=A\left(T^{n-1} x\right) \ldots A(T x) A(x)$. 
When the base map $T$ is fixed we refer to the matrix valued function $A: X \rightarrow \operatorname{Mat}_{m}(\mathbb{R})$ as being the linear cocycle.

The repeated Lyapunov exponents (LE) of the cocycle $A$ are denoted by $L_{1}(A) \geq L_{2}(A) \geq \ldots \geq L_{m}(A) \geq-\infty$. By Kingman's ergodic theorem, they are the pointwise $\mu$-almost everywhere and average limits

$$
L_{k}(A)=\lim _{n \rightarrow+\infty} \frac{1}{n} \log s_{k}\left(A^{(n)}(x)\right)=\lim _{n \rightarrow+\infty} \int_{X} \frac{1}{n} \log s_{k}\left(A^{(n)}(x)\right) d \mu(x),
$$

where $s_{k}(g)$ stands for the $k$-th singular value of a matrix $g \in \operatorname{Mat}_{m}(\mathbb{R})$.

In particular, $L_{1}(A)$, the top Lyapunov exponent of $A$, is the limit as $n \rightarrow+\infty$ of the finite scale top Lyapunov exponents

$$
L_{1}^{(n)}(A):=\int_{X} \frac{1}{n} \log \left\|A^{(n)}(x)\right\| d \mu(x) .
$$

We say that a LE is simple when its multiplicity is one, that is, when it is distinct from all the other LE. When all LE of a cocycle $A$ are simple, we say that $A$ has simple Lyapunov spectrum.

A quasi-periodic cocycle is a linear cocycle over some ergodic torus translation on a finite dimensional torus $\mathbb{T}^{d}=(\mathbb{R} / \mathbb{Z})^{d}$ equipped with the Haar measure (which we denote by $|\cdot|$ ).

In this paper we study analytic quasi-periodic cocycles, that is, cocycles in the Banach space $C_{r}^{\omega}\left(\mathbb{T}^{d}, \operatorname{Mat}_{m}(\mathbb{R})\right)$ of all analytic functions $A: \mathbb{T}^{d} \rightarrow \operatorname{Mat}_{m}(\mathbb{R})$ having a holomorphic, continuous up to the boundary extension to $\mathcal{A}_{r}^{d}=\mathcal{A}_{r} \times \ldots \times \mathcal{A}_{r} \subset \mathbb{C}^{d}$, where we denote by $\mathcal{A}_{r}:=$ $\{z \in \mathbb{C}: 1-r<|z|<1+r\}$ the annulus of width $2 r$ around the torus $\mathbb{T}$. We endow this space with the uniform norm $\|A\|_{r}:=\sup _{z \in \mathcal{A}_{r}^{d}}\|A(z)\|$.

The main result of this paper is a uniform large deviation type estimate for the iterates of any analytic quasi-periodic cocycle with simple top Lyapunov exponent. We refer to such a result as a uniform fiber LDT estimate. Our method requires a generic arithmetic assumption on the translation vector.

A fiber LDT estimate for a cocycle $A$ over some base dynamics $(X, \mu, T)$ has the form

$$
\mu\left\{x \in X:\left|\frac{1}{n} \log \left\|A^{(n)}(x)\right\|-L_{1}^{(n)}(A)\right|>\epsilon\right\}<\iota(n, \epsilon),
$$

where $\epsilon>0$ is small and $\iota(n, \epsilon) \rightarrow 0$ fast as $n \rightarrow \infty$.

We call such an estimate uniform when the rate function $\iota$ is stable under small perturbations of the cocycle $A$.

Establishing statistical properties like large deviation estimates is a difficult problem for most dynamical systems. The first results of this kind for quasi-periodic base dynamics were obtained by J. Bourgain and 
M. Goldstein [7] and by M. Goldstein and W. Schlag [17] in the context of a one-parameter family of $\mathrm{SL}_{2}(\mathbb{R})$-valued cocycles corresponding to a lattice Schrödinger operator.

The cocycles considered here are $\operatorname{Mat}_{m}(\mathbb{R})$-valued. We distinguish between identically singular (i.e. non-invertible anywhere) and nonidentically singular cocycles. We studied the latter in Chapter 6 of our monograph [14. This paper is concerned with the former, which presents significant technical challenges, especially in the several variables case $d>1$.

The idea is to first prove a non-uniform fiber LDT for any identically singular cocycle, through reduction to a maximal rank (hence nonidentically singular) cocycle of a lesser dimension. The uniform LDT estimate will then be derived by induction on the number of iterates, with the base step provided by the non-uniform statement.

This result is interesting in itself and for its subsequent applications; moreover, the method developed here to derive it might also prove useful in other contexts.

The next result of this paper concerns the continuity of the Lyapunov exponents at any cocycle in $C_{r}^{\omega}\left(\mathbb{T}^{d}, \operatorname{Mat}_{m}(\mathbb{R})\right)$. The result is quantitative, in that it also provides an explicit (weak-Hölder) modulus of continuity locally near cocycles with simple Lyapunov exponents. These statements follow from the abstract continuity theorem (ACT) obtained in Chapter 3 of our monograph [14], which is applicable in this context once the uniform fiber LDT is proven.

The continuity theorem is then used to derive criteria for the positivity and the simplicity of the Lyapunov exponents for quasi-periodic cocycles. This in turn leads to optimal lower bounds on Lyapunov exponents and on the gaps between consecutive Lyapunov exponents associated with discrete, quasi-periodic band lattice Schrödinger operators (also referred to as block Jacobi operators).

We note that (in addition to its intrinsic interest) treating the case of identically singular cocycles is exactly what allows us to derive the aforementioned consequences on the positivity and simplicity of the LE. More precisely, for cocycles with a certain structure, using a simple limiting argument, we obtain asymptotic formulas for the LE, which, under appropriate assumptions, imply lower bounds on LE or on the gaps between consecutive LE. A simple illustration of this, regarding the LE associated with discrete Schrödinger operators, is given in Proposition 1.1. 
Our method applies equally to cocycles on the 1 -variable torus $\mathbb{T}$ and on a several variables torus $\mathbb{T}^{d}$ with $d>1$. There are currently more and sharper results available for the former model. Let us now emphasize the differences between the analysis of the case $d=1$ and that of the case $d>1$ and explain how most of the one-variable arguments cannot be applied in the several variables setting.

In [28] E. Sorets and T. Spencer considered the quasi-periodic Schrödinger cocycle

$$
A_{\lambda, E}(x)=\left[\begin{array}{cr}
\lambda f(x)-E & -1 \\
1 & 0
\end{array}\right]
$$

where $f$ is a (fixed) real analytic and non-constant function on the 1 -variable torus $\mathbb{T}$.

They proved that the top LE of this cocycle is bounded from below by $\frac{1}{2} \log |\lambda|$ for all $E \in \mathbb{R}$ (and also for all translations $\omega$ ) provided that $|\lambda| \geq \lambda_{0}$, where $\lambda_{0}$ depends only on $f$. It is important in applications to spectral theory problems for the corresponding lattice Schrödinger operator that the lower bound on the LE and the threshold $\lambda_{0}$ be uniform in the energy parameter $E$.

The idea of the proof is to complexify the analytic function $f(x)$ to a neighborhood of $\mathbb{T}$, and then to use the fact that the equation $f(z)-s=0$ has finitely many zeros in a compact set, for any given $s \in \mathbb{R}$. This is due precisely to the fact that $f$ is a one variable holomorphic, non-constant function.

That neighborhood of the torus contains an annulus $\mathcal{A}_{r}$, which we identify with a strip $[0,1] \times[-r, r]$. Then for most $y \in[-r, r]$, i.e. for most horizontal lines, the equation $f(x+i y)-\frac{E}{\lambda}=0$ has no solutions in $x$. Writing for such $y$

$$
A_{\lambda, E}(x+i y)=\left[\begin{array}{cr}
\lambda f(x+i y)-E & -1 \\
1 & 0
\end{array}\right]=\left[\begin{array}{cr}
\lambda\left(f(x+i y)-\frac{E}{\lambda}\right) & -1 \\
1 & 0
\end{array}\right],
$$

it is then clear that the cocycle $A_{\lambda, E}(\cdot+i y)$ is uniformly hyperbolic, provided we choose $\lambda$ large enough. In particular this ensures a lower bound on the top LE of the cocycles $A_{\lambda, E}(\cdot+i y)$, for most $y \in[-r, r]$.

The challenge is then to transfer such a lower bound to $A_{\lambda, E}(x)$, that is, to $y=0$. E. Sorets and T. Spencer accomplish this by proving an extension of Jensen's formula to meromorphic functions; an alternative argument given by J. Bourgain (see [5]) uses harmonic measure; in [12] we gave a simple argument, based on Hardy's convexity theorem (see [15]), that applies to general higher dimensional analytic cocycles on $\mathbb{T}$. In all of these arguments, the crucial ingredient is the fact that 
the function $u_{A}^{(n)}(x):=\frac{1}{n} \log \left\|A^{(n)}(x)\right\|$ has a subharmonic extension to $\mathcal{A}_{r}$, which is due to $A(z)$ being a holomorphic function on $\mathcal{A}_{r}$.

To summarize, positivity of the top LE for analytic quasi-periodic cocycles on $\mathbb{T}$ for large $\lambda$ (which is a necessary condition) follows from the following key ingredients: the set $\{z: f(z)-s=0\}$ has "low algebraic complexity", hence most orbits of the translation will avoid it; and, essentially, the convexity of the map $y \mapsto L_{1}\left(A_{\lambda, E}(\cdot+i y)\right)$. We emphasize that the former is not available in the several variables $(d>1)$ torus translation setting.

We note the fact that the lower bounds on the top LE obtained in [28, 5, 12] in the one-frequency $(d=1)$ setting are uniform in the frequency. Furthermore, Z. Zhang [29] obtained a sharp (and uniform) lower bound for the top LE of the Schrödinger cocycle (1.2), while very recently, R. Han and C. Marx [20] obtained a precise (and uniform) asymptotic formula for the top LE of the same cocycles. When $d>1$, the only lower bound available which is uniform in the frequency is due to J. Bourgain [6], and it applies to the several variables analogue of the Schrödinger cocycle 1.2 .

Let us now discuss the continuity of the LE for quasi-periodic cocycles on $\mathbb{T}$. J. Bourgain and S. Jitormiskaya [8] proved joint continuity in $(E, \omega) \in \mathbb{R} \times(\mathbb{R} \backslash \mathbb{Q})$ of the LE of the Schrödinger cocycle 1.2 (this particular result was in fact obtained by J. Bourgain in [6] for $\mathbb{T}^{d}$ with $d>1$ as well). S. Jitomirskaya and C. Marx proved joint continuity in cocycle and frequency for $\mathrm{Mat}_{2}(\mathbb{C})$-valued cocycles. More recently, A. Ávila, S. Jitomirskaya and C. Sadel [1] extended this result to cocycles of arbitrary dimension. More precisely, they proved continuity of all LE in $(A, \omega) \in C_{r}^{\omega}\left(\mathbb{T}, \operatorname{Mat}_{m}(\mathbb{C})\right) \times(\mathbb{R} \backslash \mathbb{Q})$. We stress that these continuity results are not quantitative, unlike the ones addressed in this work.

The proof of the result in [1] uses the fact that given an analytic function $A: \mathbb{T} \rightarrow \operatorname{Mat}_{m}(\mathbb{C})$, having a holomorphic extension to some strip of width $r>0$, the complex cocycles $A_{y}: \mathbb{T} \rightarrow \operatorname{Mat}_{m}(\mathbb{C})$, $A_{y}(x):=A(x+i y)$ have dominated splitting for most $y \in(-r, r)$. The continuity of the Lyapunov exponents for cocycles having dominated splitting comes with a soft argument from the theory of hyperbolic dynamical systems, because dominated splitting is a kind of projective hyperbolicity. Finally, the continuity of the LE for $A=A_{y=0}$ follows using the convexity of the function $y \mapsto L_{1}\left(A_{y}\right)$.

To summarize, continuity of the LE for (general, higher dimensional) analytic quasi-periodic cocycles on $\mathbb{T}(d=1)$ follows from the following 
key ingredients: the dominated splitting of certain perturbations of the cocycle and a convexity argument.

In contrast with this, we show in a separate paper that for $d>1$ there are homotopy classes of analytic functions $A: \mathbb{T}^{d} \rightarrow \mathrm{GL}_{m}(\mathbb{C})$ whose corresponding quasi-periodic cocycles do not have dominated splitting. Therefore, even if the convexity argument had a counterpart when $d>1$, the approach in [1 could not be used to establish the continuity of the LE in the several variables torus translation setting.

When $d=1$, the dominated splitting property of the cocycle $A_{y}$ for most $y$ relies essentially on the fact that the zeros of a one variable holomorphic function are isolated points.

This property does not hold for several variables analytic functions $f: \mathbb{T}^{d} \rightarrow \mathbb{C}, d>1$. A simple dimension argument shows that generically, the zero set $Z(f, y):=\left\{x \in \mathbb{T}^{d}: f(x+i y)=0\right\}$, has dimension $d-2 \geq 0$. Hence, if $d>1$, there are open sets of analytic functions $f: \mathbb{T}^{d} \rightarrow \mathbb{C}$ having holomorphic extensions to a strip of width $r>0$ which have zeros on every torus $\mathbb{T}_{y}^{d}:=\left\{x+i y: x \in \mathbb{T}^{d}\right\}$ with $y \in(-r, r)^{d}$. Thus, considering the cocycle $A(x):=\left[\begin{array}{cc}f(x) & -1 \\ 1 & 0\end{array}\right]$, even if most values of $f$ are very large, the cocycles $A_{y}(x):=A(x+i y)$ are not expected to be uniformly hyperbolic. This amounts to saying that the cocycles $A_{y}$ will not have dominated splitting.

Therefore, the zeros of the function $f$ present unavoidable technical difficulties when we address the problem of continuity of the LE of quasi-periodic cocycles over a several variables torus translation.

A more robust approach, that works for both $d=1$ and $d>1$, was introduced by M. Goldstein and W. Schlag [17] in the context of Schrödinger cocycles $A_{\lambda, E}$ like (1.2). This approach proceeds by establishing LDT estimates for the iterates of the cocycle and it uses in an essential way a deterministic result on the norm growth of long products of $\mathrm{SL}_{2}(\mathbb{R})$ matrices, which the authors call the Avalanche Principle (AP). While this method requires that the translation $\omega$ be fixed and satisfy a generic arithmetic condition, the result provides a modulus of continuity $\left.\right|^{1}$ (i.e. Hölder when $d=1$, weak-Hölder when $d>1$ ) for the top Lyapunov exponent regarded as a function of the energy parameter $E$.

\footnotetext{
${ }^{1}$ According to a private conversation of the second author with Qi Zhou, regarding the latter's yet unpublished (joint) work, an arithmetic condition is in fact necessary for establishing such a modulus of continuity.
} 
In our recent monograph [14, we extend this approach in both depth and breadth, making it applicable to any space of cocycles, of any dimension and over any base dynamics, provided appropriate LDT estimates are available in the given setting. We refer the reader to Chapters 1 and 6 of this monograph, as well as to our survey [13] for a more thorough review of related results. Moreover, we note that the recent surveys of S. Jitomirskaya and C. Marx [23] and D. Damanik [11] provide the interested reader with an excellent overview of related topics.

Finally, we comment on the simplicity of the Lyapunov exponents. In the 1980s, Y. Guivarc'h and A. Raugi [19] and I. Ya. Gol'dsheid and G. A. Margulis [16] obtained sufficient criteria for the simplicity of the LE of locally constant cocycles over a Bernoulli shift. More recently, results on this kind for other models were obtained by $\mathrm{C}$. Bonatti and M. Viana [4] and by A. Ávila and M. Viana [3, 2].

We are not aware of any previously established sufficient criteria for the simplicity of the LE for quasi-periodic models. However, in the 1-variable torus translation case, the general continuity result in [1] can be used to obtain a similar (but slightly less precise) criterion for simplicity to the one we formulate and prove here.

As mentioned earlier, the key to all the results in this paper is proving a uniform fiber LDT estimate like (1.1). The uniformity of this estimate in the cocycle represents the crucial assumption in the proof of the ACT. This is the main reason for the case of identically singular cocycles being significantly more challenging, especially when considering translations on the several variables $(d>1)$ torus. We give some details on these challenges and on how they will be overcome.

One difficulty is related to the fact that being identically singular (and hence not having full rank) is not an open condition in the space of analytic cocycles. Thus a small perturbation of such a cocycle could have a higher rank.

Given an identically singular cocycle $A$ with $L_{1}(A)>-\infty$, we show that it is semi-conjugated to a maximal rank (hence non-identically singular) cocycle of a smaller dimension; we previously established (see chapter 6 in [14]) uniform fiber LDT estimates for non-identically singular cocycles; via the semi-conjugacy relation, this leads to fiber LDT for the cocycle $A$. Incidentally we also derive the fact (which was recently independently proven in [27]) that an analytic cocycle is nilpotent if and only if its top Lyapunov exponent is $-\infty$.

We note that since the rank of $A$ may change under perturbations, the parameters of the LDT obtained through semi-conjugacy may blow 
up. Thus this argument will only provide non-uniform fiber LDT estimates for analytic cocycles. We prove a uniform fiber LDT estimate in the vicinity of an identically singular cocycle $A$ using an inductive procedure based on the avalanche principle. We explain below the mechanics of the proof.

The non-uniform LDT is used to get the procedure started-given a large enough initial scale (i.e. number of iterates) $n_{0}$, the fiber LDT estimate holds for $A$ at this scale, hence if we choose any other cocycle $B$ at a small enough distance from $A$, this estimate will transfer over to $B$ by proximity. Of course, this can only be done once, at an initial scale of order $n_{0}$, as the size of the neighborhood of $A$ depends on $n_{0}$. Therefore, we obtain an estimate of the form

$$
\left|\left\{x \in \mathbb{T}^{d}:\left|\frac{1}{n} \log \left\|B^{(n)}(x)\right\|-L_{1}^{(n)}(B)\right|>n^{-a}\right\}\right|<e^{-n^{b}},
$$

for some constants $a, b>0$, for $n \asymp n_{0}$ and for all cocycles $B$ in a small neighborhood of $A$.

The goal is to derive an estimate like (1.3) at a next scale $n_{1} \gg n_{0}$, then at a scale $n_{2} \gg n_{1}$ and so on, in such a way that the parameters $a$ and $b$ in this estimate do not change from one step to the next (to be precise, they will only stabilize from scale $n_{1}$ on).

For every scale $n$ and cocycle $B$ consider the function

$$
u_{B}^{(n)}(x):=\frac{1}{n} \log \left\|B^{(n)}(x)\right\| .
$$

The AP will essentially allow us to represent the function $u_{B}^{\left(n_{1}\right)}(x)$ corresponding to the scale $n_{1}$, in terms of certain Birkhoff averages of the function $u_{B}^{(m)}(x)$ corresponding to scales $m \asymp n_{0}$, for which the estimate (1.3) is already available. There is, of course, an error term in this representation; furthermore, this representation does not hold for all phases $x$, but only outside of a relatively small set of phases related to the exceptional set in the LDT estimate (1.3) at scales of order $n_{0}$.

By the pointwise ergodic theorem, these Birkhoff averages converge almost everywhere to the means of the corresponding observables. However, since we are performing an inductive process (or a multiscale analysis with finite steps) we need a quantitative version of this result, one where the rate of convergence and the size of the exceptional sets of phases depend explicitly on the number of iterates.

Such a quantitative Birkhoff ergodic theorem (qBET) will indeed hold due to: the arithmetic assumption on the translation $\omega$ (this takes care of some small denominators issues); and the fact that since the cocycles $B(x)$ are real analytic (hence they have holomorphic extensions), 
the functions $u_{B}^{(n)}(x)$ defined above extend to a neighborhood of $\mathbb{T}^{d}$ as separately subharmonic functions (i.e. subharmonic in each variable).

It is crucial for our purposes that the parameters that determine the qBET for the observables $u_{B}^{(n)}$ be uniform in both $B$ (in a fixed neighborhood of $A$ ) and $n$. Moreover, we also need a uniform bound on the $L^{2}$-norms of these observables - in part because there are sets of phases over which we have no control, hence an estimate on $L_{1}^{(n)}(B)=$ $\int_{\mathbb{T}^{d}} u_{B}^{(n)}(x) d x$ can be obtained from having control on $u_{B}^{(n)}(x)$ for most phases $x$ and a bound on the global $L^{2}$-norm of $u_{B}^{(n)}$. These are the two most technically challenging aspects of the proof, and the challenge comes from the fact that the cocycles $B$ may be identically singular.

The qBET for subharmonic (when $d=1$ ) or separately subharmonic (when $d>1$ ) functions $u(z)$ were proven (see for instance [5, 17, 25]) under the assumption that $|u(z)| \leq C<\infty$ throughout the domain. The parameters that determine this estimate depend only on the bound $C$ and the size of the domain, hence such a qBET applies uniformly if the observables considered are uniformly bounded.

In the context of this paper, the separately subharmonic functions $u_{B}^{(n)}(z)=\frac{1}{n} \log \left\|B^{(n)}(z)\right\|$ are clearly uniformly bounded from above. But since $B^{(n)}(z)$ may be 0 , which in the case $d>1$ may hold on an algebraically non-trivial set, these functions might not be bounded from below. However, a subharmonic function cannot be too small (i.e. too close to $-\infty$ ) for too much of the phase space, unless it were that small throughout the whole space. This is due to Cartan's estimate on logarithmic potentials, and by means of Fubini it also holds for separately subharmonic functions.

Thus, if we can establish that the functions $u_{B}^{(n)}(x)$ have a uniform lower bound at least somewhere, then each of these function could fall below a certain (low enough) threshold only on a small set of phases $x$. We horizontally truncate $u_{B}^{(n)}(z)$ from below, so the resulting function is still separately subharmonic, it is bounded from above and below (hence the qBET for bounded observables applies to it) and it agrees with $u_{B}^{(n)}$ over much of the phase space, provided the threshold for the truncation is chosen small enough. This will allow us to transfer the qBET (and other estimates) from the truncation to $u_{B}^{(n)}$. The threshold for the truncation of $u_{B}^{(n)}$ will be of the order $-n^{a}$, for some $a \in(0,1)$, hence the bound on the corresponding truncation will be of order $n^{a}$. This does produce errors of that order, but they can be easily absorbed. 
Finally, we note that the key fact that $u_{B}^{(n)}(x)$ have a uniform lower bound at least somewhere, cannot be established a-priori, but it will be obtained inductively and used to feed the next step of the induction.

Formulation of the main statements. We consider the Banach space $C_{r}^{\omega}\left(\mathbb{T}^{d}, \operatorname{Mat}_{m}(\mathbb{R})\right)$ of linear cocycles on $\mathbb{T}^{d}$ having a holomorphic extension to $\mathcal{A}_{r}^{d}$, continuous up to the boundary. This space is endowed with the uniform norm.

Given $t \in(0,1)$, we denote by $\mathrm{DC}_{t}$ the set of translation vectors $\omega \in \mathbb{R}^{d}$ satisfying the following Diophantine condition:

$$
\|k \cdot \omega\| \geq \frac{t}{|k|^{d+1}} \quad \text { for all } k \in \mathbb{Z}^{d} \backslash\{0\},
$$

where for any real number $x$ we write $\|x\|:=\min _{k \in \mathbb{Z}}|x-k|$.

The main result of this paper, from which everything else follows, is a large deviation estimate on the iterates of a cocycle in this space.

Theorem 1.1. Given $A \in C_{r}^{\omega}\left(\mathbb{T}^{d}, \operatorname{Mat}_{m}(\mathbb{R})\right)$ with $L_{1}(A)>L_{2}(A)$ and $\omega \in \mathrm{DC}_{\mathrm{t}}$, there are constants $\delta=\delta(A)>0, n_{1}=n_{1}(A, t) \in \mathbb{N}$, $a_{1}=a_{1}(d)>0, b_{1}=b_{1}(d)>0$ so that if $\|B-A\|_{r} \leq \delta$ and $n \geq n_{1}$, then

$$
\left|\left\{x \in \mathbb{T}^{d}:\left|\frac{1}{n} \log \left\|B^{(n)}(x)\right\|-L_{1}^{(n)}(B)\right|>n^{-a_{1}}\right\}\right|<e^{-n^{b_{1}}} .
$$

The crucial feature of the result above for our consequent continuity statements, and what makes its derivation most challenging, is the local uniformity of the estimates in the cocycle.

We say that a function is weak-Hölder continuous if its modulus of continuity is given by $w(h)=C e^{-c(\log (1 / h))^{b}}$, for some positive constants $C, c$ and $b$. Note that if $b=1$ then we have $w(h)=C h^{c}$, which corresponds to Hölder continuity.

We can now formulate the statement on the continuity of the Lyapunov exponents. They (and their consequences) hold under the assumption that $\omega \in \mathrm{DC}_{\mathrm{t}}$, that is, the translation vector is Diophantine.

Theorem 1.2. The map $C_{r}^{\omega}\left(\mathbb{T}^{d}\right.$, $\left.\operatorname{Mat}_{m}(\mathbb{R})\right) \ni A \mapsto L_{k}(A) \in[-\infty, \infty)$ is continuous for all $1 \leq k \leq m$.

Moreover, if for some $A$ and $k<m$ we have $L_{k}(A)>L_{k+1}(A)$, then locally near $A$ the map $C_{r}^{\omega}\left(\mathbb{T}^{d}, \operatorname{Mat}_{m}(\mathbb{R})\right) \ni B \mapsto\left(L_{1}+\ldots+L_{k}\right)(B) \in \mathbb{R}$ is weak-Hölder continuous.

Similarly to our results for non-identically singular cocycles, the Oseledets filtration and decomposition also depend continuously on the cocycle, and in fact they are weak-Hölder continuous too. Phrasing 
the most general version of this result is a bit technical, as one would firstly have to carefully describe the topology considered on the space of flag-valued functions (to which the Oseledets filtration of a cocycle belongs). To avoid these technicalities, here we formulate just a particular version of this result.

Let $A \in C_{r}^{\omega}\left(\mathbb{T}^{d}, \operatorname{Mat}_{m}(\mathbb{R})\right)$ and let $1 \leq k<m$. Assume that $L_{k}(A)>L_{k+1}(A)$. Denote by $\operatorname{Gr}_{k}\left(\mathbb{R}^{m}\right)$ the Grassmann manifold of $k$-dimensional subspaces of $\mathbb{R}^{m}$.

We define $E_{k}^{-}(A): \mathbb{T}^{d} \rightarrow \mathrm{Gr}_{m-k}\left(\mathbb{R}^{m}\right)$ to be the measurable component of the Oseledets filtration of $A$ corresponding to the Lyapunov exponents $\leq L_{k+1}(A)$, and $E_{k}^{+}(A): \mathbb{T}^{d} \rightarrow \operatorname{Gr}_{k}\left(\mathbb{R}^{m}\right)$ to be the direct sum of the components of the Oseledets decomposition corresponding to Lyapunov exponents $\geq L_{k}(A)$. With these notations we have the following statement.

Theorem 1.3. Let $A \in C_{r}^{\omega}\left(\mathbb{T}^{d}, \operatorname{Mat}_{m}(\mathbb{R})\right), 1 \leq k<m$ with $L_{k}(A)>$ $L_{k+1}(A)$. There are $\delta=\delta(A)>0$ and $\alpha=\alpha(A)>0$ such that for all $B_{1}, B_{2} \in C_{r}^{\omega}\left(\mathbb{T}^{d}, \operatorname{Mat}_{m}(\mathbb{R})\right)$ with $\left\|B_{i}-A\right\|_{r} \leq \delta, i=1,2$ we have

$\left|\left\{x \in \mathbb{T}^{d}: d\left(E_{k}^{ \pm}\left(B_{1}\right)(x), E_{k}^{ \pm}\left(B_{2}\right)(x)\right)>\left\|B_{1}-B_{2}\right\|_{r}^{\alpha}\right\}\right|<w\left(\left\|B_{1}-B_{2}\right\|_{r}\right)$,

where d refers to the distance on the Grassmann manifold and $w$ refers to a weak-Hölder modulus of continuity function.

The reader may consult our monograph [14] (see Chapter 6, Theorem 6.1) for the general result in the case of non-identically singular cocycles; the same will hold here as well.

To show the usefulness of our continuity result of the Lyapunov exponents for identically singular cocycles, we present an immediate application - the positivity of the maximal LE for Schrödinger cocycles.

Recall that a discrete, one-dimensional, quasi-periodic Schrödinger operator is an operator $H_{\lambda}(x)$ on $l^{2}(\mathbb{Z}) \ni \psi=\left\{\psi_{n}\right\}_{n \in \mathbb{Z}}$, defined by

$$
\left[H_{\lambda}(x) \psi\right]_{n}:=-\left(\psi_{n+1}+\psi_{n-1}\right)+\lambda f(x+n \omega) \psi_{n},
$$

where $\lambda \neq 0$ is a coupling constant, $f: \mathbb{T}^{d} \rightarrow \mathbb{R}$ is the potential function, $x \in \mathbb{T}^{d}$ is a phase parameter that introduces some randomness into the system and $\omega \in \mathbb{T}^{d}$ is a fixed incommensurable frequency.

We note that due to the ergodicity of the system, the spectral properties of the family of operators $\left\{H_{\lambda}(x): x \in \mathbb{T}^{d}\right\}$ are independent of $x$ almost surely. Moreover, $H_{\lambda}(x)$ is a bounded, self-adjoint operator, whose norm (and hence spectral radius) is $\leq 2+|\lambda|\|f\|_{L^{\infty}\left(\mathbb{T}^{d}\right)}$.

Consider the Schrödinger (i.e. eigenvalue) equation

$$
H_{\lambda}(x) \psi=E \psi
$$


for some energy (i.e eigenvalue) $E \in \mathbb{R}$ and state (i.e. eigenvector) $\psi=\left\{\psi_{n}\right\}_{n \in \mathbb{Z}} \subset \mathbb{R}$.

Define the associated Schrödinger cocycle as the one-parameter (in $E$ ) family $A_{\lambda, E}$, where

$$
A_{\lambda, E}(x):=\left[\begin{array}{cr}
\lambda f(x)-E & -1 \\
1 & 0
\end{array}\right] \in \mathrm{SL}(2, \mathbb{R}) .
$$

Note that the Schrödinger equation (1.5) is a second order finite difference equation. An easy calculation shows that its formal solutions are given by

$$
\left[\begin{array}{c}
\psi_{n+1} \\
\psi_{n}
\end{array}\right]=A_{\lambda, E}^{(n+1)}(x) \cdot\left[\begin{array}{c}
\psi_{0} \\
\psi_{-1}
\end{array}\right]
$$

where $A_{\lambda, E}^{(n)}(x)$ are the iterates of $A_{\lambda, E}(x)$, for all $n \in \mathbb{N}$.

Proposition 1.1. Let $\omega \in \mathrm{DC}_{t}$, let $f \in C_{r}^{\omega}\left(\mathbb{T}^{d}, \mathbb{R}\right)$ be an analytic, non-constant function, and consider the corresponding quasi-periodic Schrödinger cocycle

$$
A_{\lambda, E}(x)=\left[\begin{array}{cr}
\lambda f(x)-E & -1 \\
1 & 0
\end{array}\right] .
$$

There are $\lambda_{0}=\lambda_{0}(t, f)<\infty, c=c(f)>0$ and $b=b(d)>0$, such that if $|\lambda| \geq \lambda_{0}$ then the top Lyapunov exponent of the cocycle $A_{\lambda, E}$ has the following asymptotic behavior.

(a) If $|E| \leq 2|\lambda|\|f\|_{r}$ then

$$
L_{1}\left(A_{\lambda, E}\right)=\int_{\mathbb{T}^{d}} \log |\lambda f(x)-E| d x+\mathcal{O}\left(e^{-c(\log |\lambda|)^{b}}\right) .
$$

(b) If $|E| \geq 2|\lambda|\|f\|_{r}$ then

$$
L_{1}\left(A_{\lambda, E}\right)=\int_{\mathbb{T}^{d}} \log |\lambda f(x)-E| d x+\mathcal{O}\left(e^{-c(\log |E|)^{b}}\right) .
$$

In particular, there is $C_{0}=C_{0}(f)<\infty$ such that if $|\lambda| \geq \lambda_{0}$ then

$$
L_{1}\left(A_{\lambda, E}\right)>\log |\lambda|-C_{0} \text { for all } E \in \mathbb{R} .
$$

Proof. We first consider the case $|E| \leq 2|\lambda|\|f\|_{r}$.

Given $\delta, s \in \mathbb{R}$ define the cocycle

$$
S_{\delta, s}(x):=\left[\begin{array}{cr}
f(x)-s & -\delta \\
\delta & 0
\end{array}\right] \in \operatorname{Mat}_{2}(\mathbb{R}) .
$$

By factoring out $\lambda$ we have $A_{\lambda, E}=\lambda S_{\frac{1}{\lambda}, \frac{E}{\lambda}}$ so

$$
L_{1}\left(A_{\lambda, E}\right)=\log |\lambda|+L_{1}\left(S_{\frac{1}{\lambda}, \frac{E}{\lambda}}\right) .
$$

The map $(\delta, s) \mapsto S_{\delta, s}$ is Lipschitz. 
Moreover, for all $s \in \mathbb{R}$, the cocycle $S_{0, s}=\left[\begin{array}{cc}f(x)-s & 0 \\ 0 & 0\end{array}\right]$, which is identically singular, satisfies

$$
L_{1}\left(S_{0, s}\right)=\int_{\mathbb{T}^{d}} \log |f(x)-s| d x>-\infty
$$

(because $f$ is analytic and non-constant) and $L_{2}\left(S_{0, s}\right)=-\infty$.

In particular, $L_{1}\left(S_{0, s}\right)>L_{2}\left(S_{0, s}\right)$, so by the continuity Theorem 1.2 , the map $B \mapsto L_{1}(B)$ is locally weak-Hölder near $S_{0, s}$ for every $s \in \mathbb{R}$.

Then by compactness, there is $\delta_{0}>0$ such that the map

$$
\left[-\delta_{0}, \delta_{0}\right] \times\left[-2\|f\|_{r}, 2\|f\|_{r}\right] \ni(\delta, s) \mapsto L_{1}\left(S_{\delta, s}\right) \in \mathbb{R}
$$

is weak-Hölder continuous.

Therefore, there is a modulus of continuity function of the form $w(h)=C e^{-c\left(\log \frac{1}{h}\right)^{b}}$ such that if $|\delta| \leq \delta_{0}$ and if $|s| \leq 2\|f\|_{r}$ then

$$
\left|L_{1}\left(S_{\delta, s}\right)-L_{1}\left(S_{0, s}\right)\right| \leq w(|\delta|) \rightarrow 0 \quad \text { as } \delta \rightarrow 0 .
$$

Let $\lambda_{0}:=\frac{1}{\delta_{0}}$. Then for all $\lambda, E$ with $|\lambda| \geq \lambda_{0}$ and $|E| \leq 2|\lambda|\|f\|_{r}$, via the change of variables $\delta=\frac{1}{\lambda}, s=\frac{E}{\lambda}$, from the above we have

$$
\left|L_{1}\left(S_{\frac{1}{\lambda}, \frac{E}{\lambda}}\right)-L_{1}\left(S_{0, \frac{E}{\lambda}}\right)\right| \leq w\left(\frac{1}{|\lambda|}\right) \rightarrow 0 \quad \text { as }|\lambda| \rightarrow \infty .
$$

This then translates into

$$
\left|L_{1}\left(A_{\lambda, E}\right)-\log \right| \lambda\left|-\int_{\mathbb{T}^{d}} \log \right| f(x)-\frac{E}{\lambda}|d x| \leq w\left(\frac{1}{|\lambda|}\right),
$$

which implies (1.6a).

The second case, when $|E| \geq 2|\lambda|\|f\|_{r}$, is treated similarly-by factoring out $E$ instead. Indeed, for $\delta, s \in \mathbb{R}$, define the cocycle

$$
S_{\delta, s}(x):=\left[\begin{array}{cr}
s f(x)-1 & -\delta \\
\delta & 0
\end{array}\right] \in \operatorname{Mat}_{2}(\mathbb{R}) .
$$

By factoring out $E$ we have $A_{\lambda, E}=E S_{\frac{1}{E}, \frac{\lambda}{E}}$ so

$$
L_{1}\left(A_{\lambda, E}\right)=\log |E|+L_{1}\left(S_{\frac{1}{E}, \frac{\lambda}{E}}\right) .
$$

The cocycle $S_{0, s}$ is identically singular and for all $s \in \mathbb{R}$ we have

$$
L_{1}\left(S_{0, s}\right)=\int_{\mathbb{T}^{d}} \log |s f(x)-1| d x>-\infty
$$

(since $f$ is analytic and non-constant), while $L_{2}\left(S_{0, s}\right)=-\infty$. The continuity Theorem 1.2 is again applicable, ensuring weak-Hölder continuity of the top Lyapunov exponent locally near $S_{0, s}$ for every $s \in \mathbb{R}$. 
Then by a simple compactness argument, there is $\delta_{0}>0$ such that the map

$$
\left[-\delta_{0}, \delta_{0}\right] \times\left[-\frac{1}{2\|f\|_{r}}, \frac{1}{2\|f\|_{r}}\right] \ni(\delta, s) \mapsto L_{1}\left(S_{\delta, s}\right) \in \mathbb{R}
$$

has a weak-Hölder modulus of continuity $w(h)$ as before, that is,

$$
\left|L_{1}\left(S_{\delta, s}\right)-L_{1}\left(S_{0, s}\right)\right| \leq w(|\delta|)
$$

Let $\lambda_{0}:=\frac{1}{\delta_{0} 2\|f\|_{r}}$. With the change of coordinates $\delta=\frac{1}{E}, s=\frac{\lambda}{E}$, if $|\lambda| \geq \lambda_{0}$ and $|E| \geq 2|\lambda|\|f\|_{r}$, then the estimate above applies and we have

$$
\left|L_{1}\left(S_{\frac{1}{E}, \frac{\lambda}{E}}\right)-L_{1}\left(S_{0, \frac{\lambda}{E}}\right)\right| \leq w\left(\frac{1}{|E|}\right) \rightarrow 0 \quad \text { as }|E| \rightarrow \infty .
$$

This then translates into

$$
\left|L_{1}\left(A_{\lambda, E}\right)-\log \right| E\left|-\int_{\mathbb{T}^{d}} \log \right| \frac{\lambda}{E} f(x)-1|d x| \leq w\left(\frac{1}{|E|}\right),
$$

which implies $1.6 \mathrm{~b}$.

To derive the last estimate, first note that since $f(x)-s \not \equiv 0$ for every $s \in \mathbb{R}$, if $I \subset \mathbb{R}$ is compact, then the map

$$
I \ni s \mapsto \int_{\mathbb{T}^{d}} \log |f(x)-s| d x \in \mathbb{R}
$$

has a finite lower bound.

This is due to the Łojasiewicz inequality (we formulate it below, in Proposition 2.10, see also Remark 6.2 in [14]), which holds uniformly in a neighborhood of an analytic, non-identically zero function. Alternatively, although this is an overkill, since $\int_{\mathbb{T}^{d}} \log |f(x)-s| d x$ represents the Lyapunov exponent of the one-dimensional cocycle $f-s$, by the same continuity theorem of the Lyapunov exponents, the map above depends continuously on $s$, hence it has a finite lower bound on any compact set.

Then either we have $|E| \leq 2|\lambda|\|f\|_{r}$, so $\frac{E}{\lambda}$ is in a compact set, and applying 1.6a we have

$$
L_{1}\left(A_{\lambda, E}\right) \geq \log |\lambda|+\int_{\mathbb{T}^{d}} \log \left|f(x)-\frac{E}{\lambda}\right| d x-w\left(\frac{1}{|\lambda|}\right)>\log |\lambda|-C_{0}
$$


Or $|E| \geq 2|\lambda|\|f\|_{r}$, so for all $x \in \mathbb{T}^{d},\left|\frac{\lambda}{E} f(x)\right| \leq \frac{1}{2}$ and applying $1.6 \mathrm{~b}$ we have

$$
\begin{aligned}
L_{1}\left(A_{\lambda, E}\right) & \geq \log |E|+\int_{\mathbb{T}^{d}} \log \left|\frac{\lambda}{E} f(x)-1\right| d x-w\left(\frac{1}{|E|}\right) \\
& \geq \log |\lambda|+\log 2\|f\|_{r}+\log \frac{1}{2}-w\left(\frac{1}{|E|}\right)>\log |\lambda|-C_{0} .
\end{aligned}
$$

This completes the proof.

Remark 1.1. Having uniform lower bounds of the form $\gtrsim \log |\lambda|$ on the Lyapunov exponent of a Schrödinger cocycle with analytic potential is of course not new. M. Herman [21] and E. Sorets and T. Spencer [28] are classical results on this topic, with uniform bounds in $\omega \in \mathbb{T}$.

A similar estimate to (1.6a) (also under a Diophantine assumption on the frequency) was obtained in the case $d=1$ by J. Bourgain (see Proposition 11.31 in [5]), although the error there is less sharp/explicit.

Moreover, asymptotic formulas for the LE of 2-dimensional Jacobi cocycles over the 1-variable torus translation were obtained in [22]. And the continuity theorem in [1] for general, higher dimensional cocycles over the same 1-variable torus may be employed to the same effects.

What is new here is the precision of the estimates $(1.6)$, especially in the case of $f \in C_{r}^{\omega}\left(\mathbb{T}^{d}, \mathbb{R}\right)$ with $d>1$. $2^{2}$

Next we formulate similar but more general consequences of the continuity Theorem 1.2, namely some sufficient criteria for the positivity and simplicity of the LE of a quasi-periodic cocycle.

Theorem 1.4. Given $\omega \in \mathrm{DC}_{t}$ and dimensions $1 \leq l<m$, for every $\lambda \neq 0$ consider a cocycle $A_{\lambda} \in C_{r}^{\omega}\left(\mathbb{T}^{d}, \operatorname{Mat}_{m}(\mathbb{R})\right)$ with a block structure

$$
A_{\lambda}=\left[\begin{array}{cc}
\lambda M & N \\
P & Q
\end{array}\right]
$$

where $M$ is a cocycle of dimension l, i.e. $M \in C_{r}^{\omega}\left(\mathbb{T}^{d}, \operatorname{Mat}_{l}(\mathbb{R})\right)$.

There is $\lambda_{0}=\lambda_{0}\left(t, M,\|N\|_{r},\|P\|_{r},\|Q\|_{r}\right)<\infty$ such that for all $|\lambda| \geq \lambda_{0}$ the following hold.

(a) If $M$ is non identically singular, i.e. if $\operatorname{det}[M(x)] \not \equiv 0$, then there is $C_{0}=C_{0}(M)<\infty$ such that

$$
L_{l}\left(A_{\lambda}\right)>\log |\lambda|-C_{0} .
$$

(b) If all Lyapunov exponents of $M$ are simple, then the l largest Lyapunov exponents of $A_{\lambda}$ are also simple.

\footnotetext{
${ }^{2}$ When $d=1$, an even more precise estimate, which is moreover uniform in the frequency, was very recently obtained in [20].
} 
In fact, a precise estimate on the gap between consecutive Lyapunov exponents holds in this case. There are positive constants $c=c(M)$ and $b=b(d)$ such that for all $1 \leq k \leq l$,

$$
\begin{aligned}
L_{k}\left(A_{\lambda}\right) & =\log |\lambda|+L_{k}(M)+\mathcal{O}\left(e^{-c(\log |\lambda|)^{b}}\right), \text { so } \\
L_{k}\left(A_{\lambda}\right)-L_{k+1}\left(A_{\lambda}\right) & =L_{k}(M)-L_{k+1}(M)+\mathcal{O}\left(e^{-c(\log |\lambda|)^{b}}\right)
\end{aligned}
$$

for all $k<l$.

Remark 1.2. By factoring out $\lambda$, the cocycle in the above theorem can be written as $A_{\lambda}=\lambda S_{\frac{1}{\lambda}}$, where $S_{\delta}:=\left[\begin{array}{cc}M & \delta N \\ \delta P & \delta Q\end{array}\right]$.

Since as $\delta \rightarrow 0, S_{\delta} \rightarrow\left[\begin{array}{cc}M & 0 \\ 0 & 0\end{array}\right]$, by the continuity Theorem 1.2 we have that for all $1 \leq k \leq l, L_{k}\left(S_{\delta}\right) \approx L_{k}(M)$ as $\delta \rightarrow 0$.

Then $L_{k}\left(A_{\lambda}\right) \approx \log |\lambda|+L_{k}(M)$ as $|\lambda| \rightarrow \infty$, so if $k<l$ we also have

$$
L_{k}\left(A_{\lambda}\right)-L_{k+1}\left(A_{\lambda}\right) \approx L_{k}(M)-L_{k+1}(M) \quad \text { as }|\lambda| \rightarrow \infty .
$$

This says that whatever the multiplicity of a Lyapunov exponent of the block $M$, if $|\lambda| \gg 1$ then the multiplicity of the corresponding Lyapunov exponent of $A_{\lambda}$ is the same or lesser.

Item (b) in Theorem 1.4 gives a precise estimate on the gap size between consecutive Lyapunov exponents when they are all simple.

As suggested already by Proposition 1.1, in applications to mathematical physics problems it is useful to have a lower bound on the Lyapunov exponents which is uniform with respect to an extra parameter. That parameter corresponds to the energy $E$ of a Schrödinger-like family of cocycles.

Below we formulate such a result on the uniform positivity of the Lyapunov exponents for a one-parameter family of higher dimensional cocycles. This uniform bound will then be applicable to the Lyapunov exponents associated with (band lattice) Schrödinger or Jacobi operators (see Section 6).

Theorem 1.5. Given $\omega \in \mathrm{DC}_{t}$ and dimensions $1 \leq l<m$, consider the family of cocycles $A_{\lambda, E} \in C_{r}^{\omega}\left(\mathbb{T}^{d}\right.$, $\left.\operatorname{Mat}_{m}(\mathbb{R})\right)$ with a block structure

$$
A_{\lambda, E}=\left[\begin{array}{cc}
M_{\lambda, E} & N \\
P & Q
\end{array}\right]
$$

where the parameters $\lambda \neq 0, E \in \mathbb{R}$ and the block $M_{\lambda, E}$ has the form

$$
M_{\lambda, E}(x)=U(x)(\lambda F(x)+R(x)-E I)
$$


for some $U, F, R \in C_{r}^{\omega}\left(\mathbb{T}^{d}, \operatorname{Mat}_{l}(\mathbb{R})\right)$, and with $I \in \operatorname{Mat}_{l}(\mathbb{R})$ denoting the identity matrix.

We assume that $U$ is non-identically singular and that $F$ has no constant eigenvalues, i.e. $\operatorname{det}[U(x)] \not \equiv 0$ and $\operatorname{det}[F(x)-s I] \not \equiv 0$ for all $s \in \mathbb{R}$.

There are constants $\lambda_{0}=\lambda_{0}\left(t, U, F,\|R\|_{r},\|N\|_{r},\|P\|_{r},\|Q\|_{r}\right)<\infty$ and $C_{0}=C_{0}(U, F)<\infty$ such that if $|\lambda| \geq \lambda_{0}$ then

$$
L_{l}\left(A_{\lambda, E}\right)>\log |\lambda|-C_{0} \quad \text { for all } E \in \mathbb{R} .
$$

In Section 6 we specialize to cocycles associated to block Jacobi operators. These types of operators generalize in different ways the Schrödinger operator (1.4) and they may in some sense be regarded as approximations of higher dimensional discrete Schrödinger operators.

The results above imply the continuity of the Lyapunov exponents of such an operator. They also provide (for a large enough coupling constant) optimal lower bounds on its non-negative (i.e. the first half) Lyapunov exponents, sufficient criteria for their simplicity and weakHölder continuity of its integrated density of states.

By analogy to discrete Schrödinger operators like (1.4), the availability of these properties could prove crucial in the further study of the spectral properties of block Jacobi operators.

The rest of the paper is organized as follows.

In Section 2 we prove non uniform fiber LDT estimates for identically singular cocycles (Theorem 2.1). For that we show that such cocycles are semi-conjugated to maximal rank cocycles (Lemma 2.9). This in turn allows us to relate the pointwise finite scale LE of an identically singular cocycle with those of its maximal rank reduction (Propositions 2.13 and 2.16).

In Section 3 we prove a quantitative Birkhoff ergodic theorem (Theorem 3.1 for functions with a separately subharmonic extension to $\mathcal{A}_{r}^{d}$, under the weaker assumption of having a lower bound only at some point on $\mathbb{T}^{d}$.

In Section 4 we prove our main result, on the existence of uniform fiber LDT estimates for identically singular cocycles (Theorem 4.1).

In Section 5 we prove Theorems 1.2 and 1.3 on the continuity of the Lyapunov exponents and of the Oseledets filtration. Then we prove Theorems 1.4 and 1.5 on the positivity and simplicity of LE for certain families of cocycles.

Finally, in Section 6 we apply the previous results to block Jacobi operators (Theorem 6.1 and Corollary 6.1. 


\section{THE PROOF OF THE NON-UNIFORM FIBER LDT}

In a previous work (see [14]), we established (uniform) fiber LDT estimates for analytic cocycles $R(x)$ that are non identically singular, in the sense that $\operatorname{det}[R(x)] \not \equiv 0$.

The goal of this section is to establish a non-uniform fiber LDT estimate for identically singular cocycles $A \in C_{r}^{\omega}\left(\mathbb{T}^{d}, \operatorname{Mat}_{m}(\mathbb{R})\right)$ with $L_{1}(A)>-\infty$. This is obtained by means of semi-conjugating the iterates of $A(x)$ with those of a reduced maximal rank cocycle $R_{A}(x)$. Having maximal rank, the reduced cocycle $R_{A}(x)$ is non-identically singular, hence it satisfies a fiber LDT estimate, and the semi-conjugacy relation allows us to transfer over the LDT estimate to $A(x)$. We note that this approach is not stable under perturbations of the original cocycle $A(x)$, hence it cannot provide a uniform fiber LDT estimate.

Some linear algebra considerations. Let $1 \leq k \leq m$ and consider a matrix $V \in \operatorname{Mat}_{k \times m}(\mathbb{R})$ with rank $k$, which is equivalent to saying that $\operatorname{det}\left(V V^{T}\right) \neq 0$. The matrix $V$ determines a linear map $V: \mathbb{R}^{m} \rightarrow \mathbb{R}^{k}$. Let $V^{+}: \mathbb{R}^{k} \rightarrow \mathbb{R}^{m}$ be the corresponding pseudo-inverse. Geometrically we take $W=\operatorname{Ker}(V)^{\perp}$, so that $\left.V\right|_{W}: W \rightarrow \mathbb{R}^{k}$ is an isomorphism and set

$$
V^{+}:=\left(\left.V\right|_{W}\right)^{-1}: \mathbb{R}^{k} \rightarrow W \subset \mathbb{R}^{m} .
$$

The next proposition shows that this definition matches the usual Moore-Penrose pseudo-inverse.

Lemma 2.1. Given $V \in \operatorname{Mat}_{k \times m}(\mathbb{R})$ with rank $k$,

$$
V^{+}=V^{T}\left(V V^{T}\right)^{-1} .
$$

Proof. Since $V V^{T} \in \operatorname{Mat}_{k}(\mathbb{R})$ is a positive symmetric matrix, it admits an orthonormal basis of eigenvectors $v_{j}, j=1, \ldots, k$ such that $V V^{T} v_{j}=\lambda_{j} v_{j}$ with $\lambda_{j}>0$. Hence $\left(V V^{T}\right)^{-1} v_{j}=\lambda_{j}^{-1} v_{j}$ and for all $j=1, \ldots, k$

$$
V\left[V^{T}\left(V V^{T}\right)^{-1} v_{j}\right]=\lambda_{j}^{-1} V V^{T} v_{j}=v_{j} .
$$

Now, because $V^{T}\left(V V^{T}\right)^{-1} v_{j} \in W$, it follows that for all $j=1, \ldots, k$

$$
V^{+} v_{j}=V^{T}\left(V V^{T}\right)^{-1} v_{j} .
$$

Because these vectors form a basis of $\mathbb{R}^{k}$ the identity follows.

Lemma 2.2. If $V \in \operatorname{Mat}_{k \times m}(\mathbb{R})$ has rank $k$, then

$$
\frac{1}{\|V\|} \leq\left\|V^{+}\right\| \leq \sqrt{\frac{\left\|\operatorname{adj}\left(V V^{T}\right)\right\|}{\operatorname{det}\left(V V^{T}\right)}}
$$


where adj denotes the adjugate of a matrix, i.e. the transpose of its cofactor matrix.

Proof. The first inequality follows from the fact that $V^{+}$is a right inverse of $V: I=V V^{+}$.

For the second, using the previous lemma, for any unit vector $x$

$$
\begin{aligned}
\left\|V^{+} x\right\|^{2} & =\left\|V^{T}\left(V V^{T}\right)^{-1} x\right\|^{2} \\
& =x^{T}\left(V^{T}\left(V V^{T}\right)^{-1}\right)^{T} V^{T}\left(V V^{T}\right)^{-1} x \\
& =x^{T}\left(V V^{T}\right)^{-T}\left(V V^{T}\right)\left(V V^{T}\right)^{-1} x \\
& =x^{T}\left(V V^{T}\right)^{-1} x=\frac{1}{\operatorname{det}\left(V V^{T}\right)} x^{T} \operatorname{adj}\left(V V^{T}\right) x \\
& \leq \frac{\left\|\operatorname{adj}\left(V V^{T}\right)\right\|}{\operatorname{det}\left(V V^{T}\right)} .
\end{aligned}
$$

Given $g \in \operatorname{Mat}_{m}(\mathbb{R})$ denote by $\operatorname{rank}(g), \operatorname{Ker}(g)$ and Range $(g)$ respectively the rank, kernel and range of $g$. Let $\pi_{g} \in \operatorname{Mat}_{m}(\mathbb{R})$ denote the orthogonal projection onto Range $(g)$.

Lemma 2.3. If $g_{2}, g_{1}, g_{0} \in \operatorname{Mat}_{m}(\mathbb{R})$ are such that $\operatorname{rank}\left(g_{2} g_{1}\right)=\operatorname{rank}\left(g_{1}\right)=$ $\operatorname{rank}\left(g_{1} g_{0}\right)=k$, then

(a) $\operatorname{rank}\left(g_{2} g_{1} g_{0}\right)=k$.

(b) $g_{2} g_{1} g_{0}$ maps Range $\left(g_{0}^{T} g_{1}^{T}\right)$ isomorphically onto Range $\left(g_{2} g_{1}\right)$.

Proof. Because $\operatorname{rank}\left(g_{2} g_{1}\right)=\operatorname{rank}\left(g_{1}\right)$ we have $\operatorname{Range}\left(g_{1}^{T} g_{2}^{T}\right)=\operatorname{Range}\left(g_{1}^{T}\right)$. Hence

$$
\begin{aligned}
\operatorname{Range}\left(g_{0}^{T} g_{1}^{T}\right) & =g_{0}^{T} \operatorname{Range}\left(g_{1}^{T}\right)=g_{0}^{T} \operatorname{Range}\left(g_{1}^{T} g_{2}^{T}\right) \\
& =\operatorname{Range}\left(g_{0}^{T} g_{1}^{T} g_{2}^{T}\right)
\end{aligned}
$$

which implies (a). In fact

$$
\begin{aligned}
\operatorname{rank}\left(g_{2} g_{1} g_{0}\right) & =\operatorname{rank}\left(g_{0}^{T} g_{1}^{T} g_{2}^{T}\right)=\operatorname{dim} \operatorname{Range}\left(g_{0}^{T} g_{1}^{T} g_{2}^{T}\right) \\
& =\operatorname{dim} \operatorname{Range}\left(g_{0}^{T} g_{1}^{T}\right)=\operatorname{rank}\left(g_{1} g_{0}\right)=k .
\end{aligned}
$$

Conclusion 2.1 applied to the triplet of matrices $g_{0}^{T}, g_{1}^{T}, g_{2}^{T}$ gives

$$
\operatorname{Range}\left(g_{2} g_{1}\right)=\operatorname{Range}\left(g_{2} g_{1} g_{0}\right) \text {. }
$$

By the fundamental theorem on homomorphisms, $g_{2} g_{1} g_{0}$ induces an isomorphism between Range $\left(g_{0}^{T} g_{1}^{T} g_{2}^{T}\right)=\operatorname{Ker}\left(g_{2} g_{1} g_{0}\right)^{\perp}$ and Range $\left(g_{2} g_{1} g_{0}\right)$. Therefore item (b) follows from (2.1) and (2.2). 
The rank of a linear cocycle. Let $A \in C_{r}^{\omega}\left(\mathbb{T}^{d}, \operatorname{Mat}_{m}(\mathbb{R})\right)$ be an analytic quasi-periodic cocycle.

Definition 2.1. We call geometric rank of $A$ the number

$$
\mathrm{r}(A):=\max _{x \in \mathbb{T}^{d}} \operatorname{rank}(A(x)) .
$$

We call rank of $A$ the limit

$$
\operatorname{rank}(A):=\lim _{n \rightarrow+\infty} \mathrm{r}\left(A^{(n)}\right) .
$$

When $\operatorname{rank}(A)=m$ the cocycle $A$ is said to have maximal rank.

Remark 2.1. The limit above exists because the sequence of geometric ranks $\mathrm{r}\left(A^{(n)}\right)$ decreases. Hence this sequence eventually stabilizes, i.e. it becomes constant.

Remark 2.2. The reader should be mindful of the notational difference between $\operatorname{rank}(A)$, which represents the rank of the cocycle $A$, and $\operatorname{rank}(A(x))$, the rank of the matrix $A(x)$.

Remark 2.3. Because of analyticity, $\left\{x \in \mathbb{T}^{d}: \operatorname{rank}(A(x))=\mathrm{r}(A)\right\}$ is open and has full measure. Hence, this is a generic set in both topological and measure theoretical sense.

Proposition 2.4. If $\mathrm{r}\left(A^{(k)}\right)=\mathrm{r}\left(A^{(k+1)}\right)$ then $\operatorname{rank}(A)=\mathrm{r}\left(A^{(k)}\right)$.

Proof. Given $x \in \mathbb{T}^{d}$ consider the triplet of matrices $A\left(T^{k+1} x\right), A^{(k)}(T x)$ and $A(x)$, whose product is equal to $A^{(k+2)}(x)$. If $x$ is generic the matrices $A^{(k)}(x), A^{(k+1)}(x), A^{(k)}(T x)$ and $A^{(k+1)}(T x)$ have maximal rank. Then by assumption

$$
\begin{aligned}
& \operatorname{rank}\left(A\left(T^{k+1} x\right) A^{(k)}(T x)\right)=\operatorname{rank}\left(A^{(k+1)}(T x)\right)=\operatorname{rank}\left(A^{(k)}(T x)\right), \\
& \operatorname{rank}\left(A^{(k)}(T x) A(x)\right)=\operatorname{rank}\left(A^{(k+1)}(x)\right)=\operatorname{rank}\left(A^{(k)}(x)\right) .
\end{aligned}
$$

Hence by Lemma 2.3 ,

$$
\operatorname{rank}\left(A^{(k+2)}(x)\right)=\operatorname{rank}\left(A\left(T^{k+1} x\right) A^{(k)}(T x) A(x)\right)=\operatorname{rank}\left(A^{(k)}(x)\right) .
$$

This proves that $\mathrm{r}\left(A^{(k+2)}\right)=\mathrm{r}\left(A^{(k)}\right)$. By induction $\mathrm{r}\left(A^{(n)}\right)=\mathrm{r}\left(A^{(k)}\right)$ for all $n \geq k$.

Corollary 2.5. The geometric rank $\mathrm{r}\left(A^{(k)}\right)$ stabilizes after some order $k \leq m$. In particular, $A^{(m)}$ has geometric rank equal to $\operatorname{rank}(A)$ and so does $A^{(n)}$ for any $n \geq m$.

Definition 2.2. A cocycle $A \in C_{r}^{\omega}\left(\mathbb{T}^{d}, \operatorname{Mat}_{m}(\mathbb{R})\right)$ is said to be nilpotent when for some $n \geq 1, A^{(n)} \equiv 0$. 
Remark 2.4. The only cocycle with zero geometric rank is the constant zero cocycle. The cocycles with zero rank are exactly the nilpotent ones.

Remark 2.5. If $\operatorname{rank}(A)=k$ then by the analyticity of $A$ the set of points $x \in \mathbb{T}^{d}$ where $\operatorname{rank}\left(A^{(m)}(x)\right)=k$ is open and dense with full measure.

The following elementary proposition relates the geometric rank with singular values and exterior powers of a cocycle $A$.

Proposition 2.6. Given $A \in C_{r}^{\omega}\left(\mathbb{T}^{d}, \operatorname{Mat}_{m}(\mathbb{R})\right)$ and $k \geq 1$ the following statements are equivalent:

(1) A has geometric rank $k$,

(2) $k=\max \left\{1 \leq j \leq m: s_{j}(A(x)) \neq 0\right.$ for some $\left.x \in \mathbb{T}^{d}\right\}$,

(3) $\wedge_{k}$ A has geometric rank 1 .

Proof. Given $g \in \operatorname{Mat}_{m}(\mathbb{R}), \operatorname{rank}(g)=\max \left\{1 \leq j \leq m: s_{j}(g)>0\right\}$. This implies that $(1) \Leftrightarrow(2)$.

On the other hand,

$$
\begin{aligned}
& s_{1}\left(\wedge_{k} g\right)=s_{1}(g) s_{2}(g) \cdots s_{k}(g), \\
& s_{2}\left(\wedge_{k} g\right)=s_{1}(g) s_{2}(g) \cdots s_{k-1}(g) s_{k+1}(g) .
\end{aligned}
$$

Hence, $s_{1}(g) \geq \ldots \geq s_{k}(g)>s_{k+1}(g)=0$ if and only if $s_{1}\left(\wedge_{k} g\right)>$ $s_{2}\left(\wedge_{k} g\right)=0$. This shows that $(1) \Leftrightarrow(3)$.

Semi-conjugation to a maximal rank cocycle. Consider a linear cocycle $A \in C_{r}^{\omega}\left(\mathbb{T}^{d}\right.$, $\left.\operatorname{Mat}_{m}(\mathbb{R})\right)$ with rank $k \geq 1$ and a function $V_{A}$ : $\mathbb{T}^{d} \rightarrow \operatorname{Mat}_{k \times m}(\mathbb{R})$ consisting of $k$ rows of $A^{(m)}$ chosen so that

$$
\Omega_{A}:=\left\{x \in \mathbb{T}^{d}: \operatorname{rank}\left(V_{A}(x)\right)=k\right\}
$$

is an open set with full measure in $\mathbb{T}^{d}$. Note that

$$
\operatorname{rank}\left(V_{A}(x)\right)=k \Leftrightarrow \operatorname{det}\left(V_{A}(x) V_{A}(x)^{T}\right) \neq 0 .
$$

Let $V_{A}^{+}(x)$ be the pseudo-inverse of $V_{A}(x)$, which is well-defined for $x \in \Omega_{A}$. By Lemma 2.1, for every $x \in \Omega_{A}$,

$$
V_{A}^{+}(x)=V_{A}(x)^{T}\left(V_{A}(x) V_{A}(x)^{T}\right)^{-1} .
$$

Define $W_{A}(x)=\operatorname{Range}\left(A^{(m)}(x)^{T}\right)=\operatorname{Range}\left(V_{A}(x)^{T}\right)=\operatorname{Range}\left(V_{A}^{+}(x)\right)$ and $P_{A}(x)$ as the orthogonal projection onto $W_{A}(x)$, i.e., $P_{A}(x)=$ 
$\pi_{A^{(m)}(x)^{T}}$. By definition, for all $x \in \Omega_{A}$,

$$
\begin{aligned}
& P_{A}(x)=V_{A}^{+}(x) V_{A}(x), \\
& \mathbb{R}^{m}=W_{A}(x) \oplus^{\perp} \operatorname{Ker}\left(A^{(m)}(x)\right), \\
& A^{(n)}(x) P_{A}(x)=A^{(n)}(x) \quad \text { for all } n \geq m .
\end{aligned}
$$

For each $x \in \mathbb{T}^{d}$ let $\delta_{A, i}(x)$ be the $i$-th leading principal minor of $V_{A}(x) V_{A}(x)^{T}$, i.e., the sub-determinant corresponding to the first $i$ rows and columns of $V_{A}(x) V_{A}(x)^{T}$, and then set

$$
\begin{aligned}
g_{A}(x) & :=\prod_{j=1}^{k} \delta_{A, j}(x), \\
h_{A}(x) & :=\delta_{A, k}(x) g_{A}(T x)^{3} \\
\widetilde{A}(x) & :=h_{A}(x) P_{A}(T x) A(x) .
\end{aligned}
$$

Given $n \geq m$, since Range $\left(A^{(n)}(x)^{T}\right) \subseteq \operatorname{Range}\left(A^{(m)}(x)^{T}\right)$ and the matrices $A^{(n)}(x)$ and $A^{(m)}(x)$ have the same rank for all $x \in \cap_{n \geq 0} T^{-n} \Omega_{A}$ it follows that for a.e. phase $x \in \mathbb{T}^{d}$

$$
W_{A}(x)=\operatorname{Range}\left(A^{(n)}(x)^{T}\right)=\operatorname{Ker}\left(A^{(n)}(x)\right)^{\perp} .
$$

Notice that $V_{A}, g_{A}$ and $h_{A}$ are analytic functions over the same analytic domain $\mathcal{A}_{r}^{d}$ as $A$.

The functions $g_{A}$ and $h_{A}$ are not identically zero. Indeed, by Sylvester's criterion for positive definiteness and the fact that $V_{A}$ has rank $k \geq 1$, we have $\delta_{A, i}(x)>0$ for all $x \in \Omega_{A}$. This implies that $g_{A}(x)>0$ and $h_{A}(x)>0$ for all $x \in \Omega_{A} \cup T^{-1} \Omega_{A}$.

Finally the following invariance relation holds.

Proposition 2.7. For all $x \in \Omega_{A} \cap T^{-1} \Omega_{A}$,

$$
\widetilde{A}(x) W_{A}(x)=W_{A}(T x) .
$$

Proof. Given $x \in \Omega_{A} \cap T^{-1} \Omega_{A}$, consider the matrices $A\left(T^{m+1} x\right), A^{(m)}(T x)$ and $A(x)$, whose product $A^{(m+2)}(x)$ has $\operatorname{rank} k=\operatorname{rank}(A)$. By (2.7) above we have

$$
A^{(m+2)}(x)=A^{(m+1)}(T x) P_{A}(T x) A(x) .
$$

By item (b) of Lemma 2.3 applied to the previous triplet of matrices, $A^{(m+2)}(x)$ maps $W_{A}(x)$ isomorphically onto $\operatorname{Range}\left(A^{(m+1)}(T x)\right)$ and, similarly, the map $A^{(m+1)}(T x)$ takes $W_{A}(T x)$ isomorphically onto Range $\left(A^{(m+1)}(T x)\right)$. Therefore the factorization (2.13) implies that the linear map $P_{A}(T x) A(x)$ sends $W_{A}(x)$ isomorphically onto $W_{A}(T x)$. 
Finally, since $h_{A}$ does not vanish over $\Omega_{A} \cap T^{-1} \Omega_{A}, \widetilde{A}(x)$ induces an isomorphism between $W_{A}(x)$ and $W_{A}(T x)$.

Definition 2.3. The reduced cocycle of $A$ is the natural extension to $\mathbb{T}^{d}$ of the mapping $R_{A}: \Omega_{A} \rightarrow \operatorname{Mat}_{k}(\mathbb{R})$,

$$
R_{A}(x):=V_{A}(T x) \widetilde{A}(x) V_{A}^{+}(x) .
$$

The next lemma ensures that $R_{A}$ extends analytically to $\mathcal{A}_{r}^{d}$.

Lemma 2.8. Assuming $\operatorname{rank}(A)=k \geq 1$, the following functions have analytic extensions to $\mathcal{A}_{r}^{d}$ :

(1) $g_{A}, h_{A}: \mathbb{T}^{d} \rightarrow \mathbb{R}$

(2) $\left(g_{A}\right)^{3} P_{A}: \mathbb{T}^{d} \rightarrow \operatorname{Mat}_{m}(\mathbb{R})$,

(3) $\widetilde{A}: \mathbb{T}^{d} \rightarrow \operatorname{Mat}_{m}(\mathbb{R})$

(4) $R_{A}: \mathbb{T}^{d} \rightarrow \operatorname{Mat}_{k}(\mathbb{R})$.

Moreover, $R_{A}$ has maximal rank, i.e., $\operatorname{det}\left(R_{A}\right) \not \equiv 0$.

Proof. Clearly $\delta_{A, i}, g_{A}, h_{A}$ and $V_{A}$ are analytic over $\mathcal{A}_{r}^{d}$.

Consider the QR-decomposition for $V_{A}(x)^{T}$. Transposing it we get $V_{A}(x)=U(x) K(x)$, where $U(x) \in \operatorname{Mat}_{k}(\mathbb{R})$ is a lower triangular matrix with 1 s along the diagonal, and $K(x) \in \operatorname{Mat}_{k \times m}(\mathbb{R})$ is orthogonal, in the sense that the rows $\kappa_{1}(x), \ldots, \kappa_{k}(x)$ of $K(x)$ are mutually orthogonal. In fact $K(x) K(x)^{T}$ is the square of the following positive diagonal matrix $D(x)=\operatorname{diag}\left(\left\|\kappa_{1}(x)\right\|, \ldots,\left\|\kappa_{k}(x)\right\|\right) . K(x)$ is obtained applying the Gram-Schmidt orthogonalization process to the rows of $V_{A}(x)$. In each step the row $v_{j}(x)$ of $V_{A}(x)$ gives rise to the row $\kappa_{j}(x)$ of the orthogonal matrix $K(x)$

$$
\kappa_{j}(x):=v_{j}(x)-\sum_{i<j} \frac{\left\langle v_{j}(x), \kappa_{i}(x)\right\rangle}{\left\|\kappa_{i}(x)\right\|^{2}} k_{i}(x) .
$$

Inductively it follows that for each $j=1, \ldots, k$,

$$
\left\|\kappa_{1}(x)\right\|^{2} \ldots\left\|\kappa_{j-1}(x)\right\|^{2} \kappa_{j}(x) \quad \text { and } \quad\left\|\kappa_{1}(x)\right\|^{2} \ldots\left\|\kappa_{j}(x)\right\|^{2}
$$

are polynomial functions of the components of the vectors $v_{1}(x), \ldots, v_{j}(x)$, and in particular they are analytic functions on $\mathcal{A}_{r}^{d}$. Since $\operatorname{det} U(x)=1$,

$$
\begin{aligned}
\delta_{A, k}(x) & =\operatorname{det}\left[V_{A}(x) V_{A}(x)^{T}\right]=\operatorname{det}\left[U(x) K(x) K(x)^{T} U(x)^{T}\right] \\
& =\operatorname{det}\left[K(x) K(x)^{T}\right]=\operatorname{det}\left[D(x)^{2}\right]=\prod_{j=1}^{k}\left\|\kappa_{j}(x)\right\|^{2} .
\end{aligned}
$$

Denoting by $\{M\}_{i}$ the sub-matrix of $M \in \operatorname{Mat}_{k}(\mathbb{R})$ having order $i$ and both indices in $\{1, \ldots, i\}$, since $U(x)$ is a lower triangular matrix, 
we have

$$
\left\{U(x) M U(x)^{T}\right\}_{i}=\{U(x)\}_{i}\{M\}_{i}\{U(x)\}_{i}^{T} .
$$

Hence, as above we obtain

$$
\delta_{A, i}(x)=\operatorname{det}\left\{U(x) K(x) K(x)^{T} U(x)^{T}\right\}_{i}=\left\|\kappa_{1}(x)\right\|^{2} \ldots\left\|\kappa_{i}(x)\right\|^{2} .
$$

Thus $g_{A} D^{-2}$ and $g_{A} K$ have analytic extensions to $\mathcal{A}_{r}^{d}$. The rows of $D(x)^{-1} K(x)$ form an orthonormal basis of $W_{A}(x)$, and the orthogonal projection onto $W_{A}(x)$ is given by

$$
\begin{aligned}
P_{A}(x) & =\left(D(x)^{-1} K(x)\right)^{T}\left(D(x)^{-1} K(x)\right) \\
& =K(x)^{T} D(x)^{-2} K(x) .
\end{aligned}
$$

It follows that $\left(g_{A}\right)^{3} P_{A}$ has an analytic extension to $\mathcal{A}_{r}^{d}$. Thus, also $\left(\delta_{A, k}\right)^{-1} \widetilde{A}=\left(g_{A} \circ T\right)^{3}\left(P_{A} \circ T\right) A$ has an analytic extension to $\mathcal{A}_{r}^{d}$. From (2.3) and (2.4) we obtain

$$
\begin{aligned}
R_{A}(x) & =V_{A}(T x) \widetilde{A}(x) V_{A}(x)^{T}\left(V_{A}(x) V_{A}(x)^{T}\right)^{-1} \\
& =\frac{1}{\operatorname{det}\left[V_{A}(x) V_{A}(x)^{T}\right]} V_{A}(T x) \widetilde{A}(x) V_{A}(x)^{T} \operatorname{adj}\left(V_{A}(x) V_{A}(x)^{T}\right) \\
& =V_{A}(T x)\left(\left(\delta_{A, k}\right)^{-1}(x) \widetilde{A}(x)\right) V_{A}(x)^{T} \operatorname{adj}\left(V_{A}(x) V_{A}(x)^{T}\right) .
\end{aligned}
$$

This formula proves that $R_{A}$ has an analytic extension to $\mathcal{A}_{r}^{d}$.

Finally, in Proposition 2.7 we have seen that $\widetilde{A}(x)$ induces an isomorphism between $W_{A}(x)$ and $W_{A}(T x)$, for all $x \in \Omega_{A} \cap T^{-1} \Omega_{A}$. But because $R_{A}(x)=V_{A}(T x) \widetilde{A}(x) V_{A}^{+}(x)$ for all $x \in \Omega_{A}$, by the definitions of $V_{A}(T x)$ and $V_{A}^{+}(x)$, we conclude that $R_{A}(x)$ induces an automorphism on $\mathbb{R}^{k}$. Hence, for all these phases $\operatorname{det} R_{A}(x) \neq 0$.

Let $h_{A}^{(n)}(x):=\prod_{i=0}^{n-1} h_{A}\left(T^{i} x\right)$ be the $n$-th iterate of the one-dimensional cocycle $h_{A} \in C_{r}^{\omega}\left(\mathbb{T}^{d}, \mathbb{R}\right)$. As mentioned earlier, $h_{A} \not \equiv 0$. The reduced cocycle $R_{A} \in C_{r}^{\omega}\left(\mathbb{T}^{d}, \operatorname{Mat}_{k}(\mathbb{R})\right)$ was shown above to be non-identically singular. The next lemma relates the iterates of the identically singular cocycle $A$ to those of the non-identically singular cocycles $R_{A}$ and $h_{A}$.

Lemma 2.9. Given $A \in C_{r}^{\omega}\left(\mathbb{T}^{d}, \operatorname{Mat}_{m}(\mathbb{R})\right)$ with rank $k \geq 1$, the following relations hold for all iterates $n \geq m$ :

$$
\begin{aligned}
& R_{A}^{(n)}(x) V_{A}(x)=V_{A}\left(T^{n} x\right) \widetilde{A}^{(n)}(x) \\
& \widetilde{A}^{(n)}(x)=h_{A}^{(n-m)}(x) \widetilde{A}^{(m)}\left(T^{n-m} x\right) A^{(n-m)}(x) \\
& A^{(n)}(x)=h_{A}^{(n-m)}(x)^{-1} A^{(m)}\left(T^{n-m} x\right) \widetilde{A}^{(n-m)}(x) .
\end{aligned}
$$


Remark 2.6. Since $m$ is fixed, the factors $\widetilde{A}^{(m)}\left(T^{n-m} x\right)$ and $A^{(m)}\left(T^{n-m} x\right)$ will essentially be negligible in future estimates.

Proof. Since the functions $V_{A}, R_{A}$ and $\widetilde{A}$ are analytic it is enough to check 2.15) for most phases $x \in \mathbb{T}^{d}$.

Consider the set of good phases

$$
G_{A}:=\bigcap_{n \geq 1} T^{-n} \Omega_{A} \cap \bigcap_{n \geq 1} T^{-n}\left\{h_{A} \neq 0\right\} .
$$

Then $G_{A}$ has full measure, and the previous relations $(2.5),(2.7),(2.11)$ and (2.12) hold for all iterates $T^{n} x$ and any phase $x \in G_{A}$.

From (2.14) and (2.5) we get

$$
R_{A}(x) V_{A}(x)=V_{A}(T x) \widetilde{A}(x) P_{A}(x)=V_{A}(T x) B(x)
$$

where $B=\widetilde{A} P_{A}$. Iterating this relation we get for all $n \geq 1$,

$$
R_{A}^{(n)}(x) V_{A}(x)=V_{A}\left(T^{n} x\right) B^{(n)}(x) .
$$

Moreover, since $B=\widetilde{A} P_{A}$ and $\left(P_{A} \circ T\right) \widetilde{A}=\widetilde{A}$,

$$
B^{(n)}(x)=\widetilde{A}^{(n)}(x) P_{A}(x) .
$$

From 2.11 and iterating Proposition 2.7, for all $n \geq m$,

$$
\operatorname{Range}\left(A^{(n)}(x)^{T}\right)=W_{A}(x)=\operatorname{Range}\left(\widetilde{A}^{(n)}(x)^{T}\right) .
$$

This implies that $\operatorname{Ker}\left(A^{(n)}(x)\right)=\operatorname{Ker}\left(\widetilde{A}^{(n)}(x)\right)$. By (2.7) we have $A^{(n)}(x)\left(I-P_{A}(x)\right)=0$. If $n \geq m$ then, since $I-P_{A}(x)$ is the orthogonal projection onto $\operatorname{Ker}\left(A^{(n)}(x)\right)=\operatorname{Ker}\left(\widetilde{A}^{(n)}(x)\right)$, we also must have $\widetilde{A}^{(n)}(x)\left(I-P_{A}(x)\right)=0$. Hence for all $n \geq m$

$$
B^{(n)}(x)=\widetilde{A}^{(n)}(x) P_{A}(x)=\widetilde{A}^{(n)}(x) .
$$

This proves 2.15).

Moreover

$$
\widetilde{A}^{(n+1)}(x)=h_{A}(x) \widetilde{A}^{(n)}(T x) P_{A}(T x) A(x)=h_{A}(x) \widetilde{A}^{(n)}(T x) A(x) .
$$

By induction, we get for all $n \geq m$

$$
\widetilde{A}^{(n)}(x)=h_{A}^{(n-m)}(x) \widetilde{A}^{(m)}\left(T^{n-m} x\right) A^{(n-m)}(x) .
$$

This proves 2.16 .

Let us now write $M_{n, m}(x):=\widetilde{A}^{(m)}\left(T^{n-m} x\right)$. From $(2.16)$ we get

$$
M_{n, m}(x) \widetilde{A}^{(n-m)}(x)=h_{A}^{(n-m)}(x) M_{n, m}(x) A^{(n-m)}(x) .
$$

The matrix $M_{n, m}(x)$ can be expressed as

$$
M_{n, m}(x)=V_{A}^{+}\left(T^{n} x\right) R_{A}^{(m)}\left(T^{n-m} x\right) V_{A}\left(T^{n-m} x\right),
$$


In fact, 2.15 implies that

$$
R_{A}^{(m)}\left(T^{n-m} x\right) V_{A}\left(T^{n-m} x\right)=V_{A}\left(T^{n} x\right) \widetilde{A}^{(m)}\left(T^{n-m} x\right) .
$$

Multiplying on the left by $V_{A}^{+}\left(T^{n} x\right)$, and using that $V_{A}^{+} V_{A}=P_{A}$ and $\left(P_{A} \circ T\right) \widetilde{A}=\widetilde{A}$ we get

$$
\begin{aligned}
V_{A}^{+}\left(T^{n} x\right) R_{A}^{(m)}\left(T^{n-m} x\right) V_{A}\left(T^{n-m} x\right) & =P_{A}\left(T^{n} x\right) \widetilde{A}^{(m)}\left(T^{n-m} x\right) \\
& =P_{A}\left(T^{n} x\right) \widetilde{A}\left(T^{n-1} x\right) \widetilde{A}^{(m-1)}\left(T^{n-m} x\right) \\
& =\widetilde{A}\left(T^{n-1} x\right) \widetilde{A}^{(m-1)}\left(T^{n-m} x\right) \\
& =\widetilde{A}^{(m)}\left(T^{n-m} x\right)=M_{n, m}(x) .
\end{aligned}
$$

Let ${ }^{3}$

$$
M_{n, m}^{+}(x):=V_{A}^{+}\left(T^{n-m} x\right) R_{A}^{(m)}\left(T^{n-m} x\right)^{-1} V_{A}\left(T^{n} x\right) .
$$

Then

$$
M_{n, m}^{+}(x) M_{n, m}(x)=V_{A}^{+}\left(T^{n-m} x\right) V_{A}\left(T^{n-m} x\right)=P_{A}\left(T^{n-m} x\right) .
$$

Thus, left multiplying (2.18) by $M_{n, m}^{+}(x)$ we get

$$
\begin{aligned}
P_{A}\left(T^{n-m} x\right) A^{(n-m)}(x) & =h_{A}^{(n-m)}(x)^{-1} P_{A}\left(T^{n-m} x\right) \widetilde{A}^{(n-m)}(x) \\
& =h_{A}^{(n-m)}(x)^{-1} \widetilde{A}^{(n-m)}(x) .
\end{aligned}
$$

Finally, left multiplying this equality by $A^{(m)}\left(T^{n-m} x\right)$ we get

$A^{(m)}\left(T^{n-m} x\right) P_{A}\left(T^{n-m} x\right) A^{(n-m)}(x)=h_{A}^{(n-m)}(x)^{-1} A^{(m)}\left(T^{n-m} x\right) \widetilde{A}^{(n-m)}(x)$.

Since $A^{(m)}\left(T^{n-m} x\right)\left(I-P_{A}\left(T^{n-m} x\right)\right)=0$ it follows that

$$
\begin{aligned}
A^{(n)}(x) & =A^{(m)}\left(T^{n-m} x\right) A^{(n-m)}(x) \\
& =A^{(m)}\left(T^{n-m} x\right) P_{A}\left(T^{n-m} x\right) A^{(n-m)}(x) \\
& =h_{A}^{(n-m)}(x)^{-1} A^{(m)}\left(T^{n-m} x\right) \widetilde{A}^{(n-m)}(x) .
\end{aligned}
$$

This proves (2.17).

$L^{2}$-boundedness and the fiber LDT estimate. We use the semiconjugation relations in Lemma 2.9 to establish non-uniform $L^{2}$-bounds and LDT estimates for the iterates of an analytic cocycle. A direct consequence of the $L^{2}$-boundedness is the fact, interesting in itself, that an analytic cocycle is nilpotent if and only if its top Lyapunov exponent is $-\infty$. This result was recently obtained independently in [27].

\footnotetext{
${ }^{3}$ Although this is not important here, the matrix $M_{n, m}^{+}(x)$ thus defined is in fact the pseudo-inverse of $M_{m, n}(x)$.
} 
A crucial tool: the Eojasiewicz inequality. We recall the following result that describes the transversality property of non-identically zero analytic functions (see for instance Lemmas 6.1 and 6.2 in [14]. This result will be used repeatedly throughout the paper.

Proposition 2.10. (Eojasiewicz inequality) Given $f \in C_{r}^{\omega}\left(\mathbb{T}^{d}, \mathbb{R}\right)$ with $f \not \equiv 0$, there are constants $S=S(f)<\infty$ and $b=b(f)>0$ such that

$$
\left|\left\{x \in \mathbb{T}^{d}:|f(x)|<t\right\}\right|<S t^{b} \quad \text { for all } t>0
$$

Furthermore, there is a constant $C=C(S, b)=C(f)<\infty$ such that

$$
\|\log |f|\|_{L^{2}\left(\mathbb{T}^{d}\right)} \leq C \text {. }
$$

We note that this result is in fact uniform in $f$, in the sense that the constants $S, b$ and $C$ are stable under small perturbations of $f$. Also, the $L^{2}$-norm may be replaced by any other $L^{p}$-norm, $1 \leq p<\infty$.

We begin with some preparatory estimates that use in an essential way the Łojasiewicz inequality.

Lemma 2.11. Let $V \in C_{r}^{\omega}\left(\mathbb{T}^{d}, \operatorname{Mat}_{k \times m}(\mathbb{R})\right)$ with $\operatorname{rank}(V(x))=k$ a.e. Then there are constants $S=S(V)<\infty$ and $c=c(V)>0$ such that for all $t>0$ we have

$$
\begin{array}{r}
\left|\left\{x \in \mathbb{T}^{d}:\left\|V^{+}(x)\right\|>\frac{1}{t}\right\}\right|<S t^{c} \\
\left|\left\{x \in \mathbb{T}^{d}:\|V(x)\|<t\right\}\right|<S t^{c}
\end{array}
$$

Proof. Since $\operatorname{rank}(V(x))=k$ for a.e. phase $x \in \mathbb{T}^{d}$, for those phases, by Lemma 2.2 we have

$$
\left\|V^{+}(x)\right\|^{2} \leq \frac{\left\|\operatorname{adj}\left[V(x) V^{T}(x)\right]\right\|}{\operatorname{det}\left[V(x) V^{T}(x)\right]} \leq \frac{C}{\operatorname{det}\left[V(x) V^{T}(x)\right]}
$$

for some constant $C=C\left(\|V\|_{L^{\infty}}, k, m\right)<\infty$.

Therefore,

$$
\left\{x:\left\|V^{+}(x)\right\|>\frac{1}{t}\right\} \subset\left\{x:\left|\operatorname{det}\left[V(x) V^{T}(x)\right]\right|<C t^{2}\right\} .
$$

The function $f(x):=\operatorname{det}\left[V(x) V^{T}(x)\right]$ is $\not \equiv 0$ on $\mathbb{T}^{d}$ (since $V(x)$ has maximal rank $k$ for a.e. $x)$ and it has an analytic extension $f(z)=$ $\operatorname{det}\left[V(z) V^{T}(z)\right]$ to $\mathcal{A}_{r}^{d}$. The Eojasiewicz inequality is then applicable to $f(x)$ and it implies

$$
\left|\left\{x \in \mathbb{T}^{d}:|f(x)| \leq C t^{2}\right\}\right| \leq S t^{c}
$$

for some constants $S=S(f)<\infty$ and $c=c(f)>0$.

Estimate 2.19) then follows from 2.21. 
By the first inequality in Lemma 2.2 , for a.e. $x \in \mathbb{T}^{d}$,

$$
\frac{1}{\left\|V^{+}(x)\right\|} \leq\|V(x)\|
$$

hence

$$
\left\{x \in \mathbb{T}^{d}:\|V(x)\|<t\right\} \subset\left\{x \in \mathbb{T}^{d}:\left\|V^{+}(x)\right\|>\frac{1}{t}\right\} .
$$

Then 2.20 follows from 2.19).

Corollary 2.12. Under the same assumptions as in the previous lemma, there are constants $S=S(V)<\infty, c=c(V)>0$ and $T_{0}=T_{0}(V)<\infty$ such that for all $T \geq T_{0}$ we have

$$
\begin{aligned}
& \left|\left\{x \in \mathbb{T}^{d}:\left|\log \left\|V^{+}(x)\right\|\right|>T\right\}\right|<S e^{-c T} \\
& \left|\left\{x \in \mathbb{T}^{d}:|\log \|V(x)\||>T\right\}\right|<S e^{-c T}
\end{aligned}
$$

In particular we have that $\log \left\|V^{+}\right\|, \log \|V\| \in L^{2}\left(\mathbb{T}^{d}\right)$.

Proof. Since for a.e. $x \in \mathbb{T}^{d}$ we have

$$
\frac{1}{\|V(x)\|} \leq\left\|V^{+}(x)\right\|
$$

it follows that

$$
\log \left\|V^{+}(x)\right\| \geq-\log \|V(x)\| \geq-\log \|V\|_{L^{\infty}}=:-T_{0} .
$$

Then if $T \geq T_{0}$, $\left\{x:\left|\log \left\|V^{+}(x)\right\|\right|>T\right\} \subset\left\{x: \log \left\|V^{+}(x)\right\|>T\right\}=\left\{x:\left\|V^{+}(x)\right\|>e^{T}\right\}$, and (2.22) follows from 2.19) after the change of variables $t:=e^{-T}$.

Estimate 2.23 follows in a similar way from 2.20). The $L^{2}$ bounds are consequences of $2.22,2.23$ ) and the fact that

$$
\int_{\mathbb{T}^{d}}|\phi(x)| d x=\int_{0}^{\infty}\left|\left\{x \in \mathbb{T}^{d}:|\phi(x)|>T\right\}\right| d T,
$$

which holds for every measurable function $\phi$.

Given any cocycle $A \in C_{r}^{\omega}\left(\mathbb{T}^{d}, \operatorname{Mat}_{m}(\mathbb{R})\right)$ of any dimension $m \geq 1$, we will use the notations $A^{(n)}(z):=A\left(T^{n-1} z\right) \ldots A(T z) A(z)$, $u_{A}^{(n)}(z):=\frac{1}{n} \log \left\|A^{(n)}(z)\right\|$ and $\left\langle u_{A}^{(n)}\right\rangle=L_{1}^{(n)}(A):=\int_{\mathbb{T}^{d}} \frac{1}{n} \log \left\|A^{(n)}(x)\right\| d x$.

By the analyticity of $A$, clearly $u_{A}^{(n)}(z)$ is separately subharmonic on $\mathcal{A}_{r}^{d}$, and if $A^{(n)} \not \equiv 0$ then $u_{A}^{(n)} \not \equiv-\infty$. 
The following statements relate the functions $u_{A}^{(n)}$ associated to the iterates of a cocycle $A$, to the corresponding functions $u_{R_{A}}^{(n)}$ and $u_{h_{A}}^{(n)}$ associated to a maximal rank cocycle $R_{A}$ and to a non-identically singular one-dimensional cocycle $h_{A}$.

Proposition 2.13. Let $A \in C_{r}^{\omega}\left(\mathbb{T}^{d}, \operatorname{Mat}_{m}(\mathbb{R})\right)$ be a quasi-periodic cocycle with $\operatorname{rank}(A)=k \geq 1$. There are a cocycle $R_{A} \in C_{r}^{\omega}\left(\mathbb{T}^{d}, \operatorname{Mat}_{k}(\mathbb{R})\right)$ with $\operatorname{det}\left[R_{A}(x)\right] \not \equiv 0$, a one dimensional cocycle $h_{A} \in C_{r}^{\omega}\left(\mathbb{T}^{d}, \mathbb{R}\right)$ with $h_{A}(x) \not \equiv 0$, a function $V_{A} \in C_{r}^{\omega}\left(\mathbb{T}^{d}, \operatorname{Mat}_{k \times m}(\mathbb{R})\right)$ with $\operatorname{rank}\left(V_{A}(x)\right)=k$ a.e. and a constant $\mathcal{C}_{A}<\infty$ such that for all phases $x \in \mathbb{T}^{d}$ and for all iterates $n \geq m$ we have:

$$
\begin{aligned}
& u_{A}^{(n)}(x) \geq \frac{n+m}{n} u_{R_{A}}^{(n+m)}(x)-u_{h_{A}}^{(n)}(x)-\frac{\mathcal{C}_{A}}{n}-\frac{1}{n} \log \left\|V_{A}^{+}(x)\right\| \\
& u_{A}^{(n)}(x) \leq \frac{n-m}{n}\left[u_{R_{A}}^{(n-m)}(x)-u_{h_{A}}^{(n-m)}(x)\right]+\frac{\mathcal{C}_{A}}{n}+\frac{1}{n} \log \left\|V_{A}^{+}\left(T^{n} x\right)\right\| .
\end{aligned}
$$

Proof. Recall that $V_{A}^{+} V_{A}=P_{A}$ by (2.5) and $V_{A} V_{A}^{+}=\mathrm{id}_{\mathbb{R}^{k}}$ by (2.4). Hence, the semi-conjugation (2.15) implies that

$$
R_{A}^{(n)}(x)=V_{A}\left(T^{n} x\right) \widetilde{A}^{(n)}(x) V_{A}^{+}(x) .
$$

Therefore, taking norms and logarithms and using that $V_{A}$ is bounded,

$$
u_{R_{A}}^{(n)}(x) \leq u_{\widetilde{A}}^{(n)}(x)+\frac{\mathcal{C}_{A}}{n}+\frac{1}{n} \log \left\|V_{A}^{+}(x)\right\| .
$$

By definition 2.10 we have $P_{A}(T x) \widetilde{A}(x)=\widetilde{A}(x)$, hence iterating, $P_{A}\left(T^{n} x\right) \widetilde{A}^{(n)}(x)=\widetilde{A}^{(n)}(x)$. Multiplying the semi-conjugation 2.15 on the left by $V_{A}^{+}\left(T^{n} x\right)$ we get

$$
\widetilde{A}^{(n)}(x)=V_{A}^{+}\left(T^{n} x\right) R_{A}^{(n)}(x) V_{A}(x) .
$$

Therefore, taking norms and logarithms in this relation

$$
u_{\widetilde{A}}^{(n)}(x) \leq u_{R_{A}}^{(n)}(x)+\frac{\mathcal{C}_{A}}{n}+\frac{1}{n} \log \left\|V_{A}^{+}\left(T^{n} x\right)\right\| .
$$

Taking norms and logarithms in 2.16 and using that $A^{(m)}$ is bounded,

$$
u_{A}^{(n)}(x) \geq \frac{n+m}{n} u_{\widetilde{A}}^{(n+m)}(x)-u_{h_{A}}^{(n)}(x)-\frac{\mathcal{C}_{A}}{n} .
$$

Similarly, taking norms and logarithms in (2.17)

$$
u_{A}^{(n)}(x) \leq \frac{n-m}{n}\left[u_{\widetilde{A}}^{(n-m)}(x)-u_{h_{A}}^{(n-m)}(x)\right]+\frac{\mathcal{C}_{A}}{n} .
$$

Combining (2.24) and (2.26) we derive the first inequality. Combining 2.25 with (2.27) we obtain the second inequality. 
Corollary 2.14. Every quasi-periodic cocycle $A \in C_{r}^{\omega}\left(\mathbb{T}^{d}, \operatorname{Mat}_{m}(\mathbb{R})\right)$ with $\operatorname{rank}(A)=k \geq 1$ is $L^{2}$-bounded, in the sense that there is $\mathrm{C}_{0}=$ $\mathrm{C}_{0}(A)<\infty$ such that for all iterates $n \geq 1$ we have

$$
\left\|u_{A}^{(n)}\right\|_{L^{2}\left(\mathbb{T}^{d}\right)} \leq \mathcal{C}_{0} \text {. }
$$

Proof. This statement was already proven for non-identically singular cocycles (see Proposition 6.3 in [14]. Therefore it applies to the maximal rank cocycle $R_{A}$ and to the one-dimensional cocycle $h_{A}$ and we have

$$
\left\|u_{R_{A}}^{(n)}\right\|_{L^{2}\left(\mathbb{T}^{d}\right)} \leq \mathcal{C}_{0} \quad \text { and } \quad\left\|u_{h_{A}}^{(n)}\right\|_{L^{2}\left(\mathbb{T}^{d}\right)} \leq \mathcal{C}_{0}
$$

for some $\mathcal{C}_{0}=\mathcal{C}_{0}(A)<\infty$ and for all $n \geq m$.

Moreover, by Corollary 2.12, $\log \left\|V_{A}^{+}\right\| \in L^{2}$ as well, so by the double estimate in Proposition 2.13, for all $n \geq m$ we have $u_{A}^{(n)} \in L^{2}$ with a uniform bound in $n$.

It remains to show that $u_{A}^{(n)} \in L^{2}\left(\mathbb{T}^{d}\right)$ also for $1 \leq n<m$.

Since $\operatorname{rank}(A) \geq 1$, for all $n \geq 1$ we have $A^{(n)}(x) \not \equiv 0$. Let $M \in$ $C_{r}^{\omega}\left(\mathbb{T}^{d}, \operatorname{Mat}_{m}(\mathbb{R})\right)$ refer to any of the iterates $A^{(n)}$ with $1 \leq n<m$. It is then enough to show that if $M(x) \not \equiv 0$ then $\log \|M(x)\| \in L^{2}\left(\mathbb{T}^{d}\right)$. At least one of the entries of $M(x)$, let us denote it by $m(x)$, must be non-identically zero. Then for all $x \in \mathbb{T}^{d}$ we have

$$
\log |m(x)| \leq \log \|M(x)\| \leq C,
$$

for some finite constant $C$.

But since $m \in C_{r}^{\omega}\left(\mathbb{T}^{d}, \mathbb{R}\right)$ and $m(x) \not \equiv 0$, by say Łojasiewicz inequality, $\log |m(x)| \in L^{2}\left(\mathbb{T}^{d}\right)$, which completes the proof.

Corollary 2.15. Given a quasi-periodic cocycle $A \in C_{r}^{\omega}\left(\mathbb{T}^{d}, \operatorname{Mat}_{m}(\mathbb{R})\right)$ the following are equivalent:

(1) $\operatorname{rank}(A)=0$,

(2) $A$ is nilpotent,

(3) $L_{1}(A)=-\infty$.

Proof. $(1) \Leftrightarrow(2)$ follows by Remark 2.4. The implication $(2) \Rightarrow(3)$ is obvious. Finally $(3) \Rightarrow(1)$ follows from Corollary 2.14 by contraposition.

Proposition 2.16. Let $A \in C_{r}^{\omega}\left(\mathbb{T}^{d}, \operatorname{Mat}_{m}(\mathbb{R})\right)$ be a quasi-periodic cocycle with $\operatorname{rank}(A)=: k \geq 1$ and fix any $a \in(0,1)$. There are a reduced (maximal rank) cocycle $R_{A} \in C_{r}^{\omega}\left(\mathbb{T}^{d}, \operatorname{Mat}_{k}(\mathbb{R})\right)$ with $\operatorname{det}\left[R_{A}(x)\right] \not \equiv 0$, a one dimensional cocycle $h_{A} \in C_{r}^{\omega}\left(\mathbb{T}^{d}, \mathbb{R}\right)$ with $h_{A}(x) \not \equiv 0$ and constants $\mathrm{C}_{0}=\mathrm{C}_{0}(A)<\infty, S=S(A)<\infty$ such that for all phases $x \in \mathbb{T}^{d}$ and all iterates $n \geq m^{6 / a}$,

$$
u_{A}^{(n)}(x)=u_{R_{A}}^{(n)}(x)-u_{h_{A}}^{(n)}(x)+r_{n}(x),
$$


where the remainder function $r_{n}(x)$ has the following properties:

(i) $\left|\left\{x \in \mathbb{T}^{d}:\left|r_{n}(x)\right|>\mathcal{C}_{0} n^{-a / 3}\right\}\right|<S e^{-n^{1-a}}$,

(ii) $\left\|r_{n}\right\|_{L^{2}\left(\mathbb{T}^{d}\right)} \leq \mathcal{C}_{0} n^{-a / 3}$.

Proof. We estimate the remainder function

$$
r_{n}(x):=u_{A}^{(n)}(x)-u_{R_{A}}^{(n)}(x)+u_{h_{A}}^{(n)}(x)
$$

from below and above using Proposition 2.13. After some simple algebraic manipulations we have:

$$
\begin{aligned}
r_{n}(x) & \geq\left[u_{R_{A}}^{(n+m)}(x)-u_{R_{A}}^{(n)}(x)\right]+\frac{m}{n} u_{R_{A}}^{(n+m)}(x)-\frac{1}{n} \log \left\|V_{A}^{+}(x)\right\|-\frac{\mathcal{C}_{A}}{n} \\
r_{n}(x) & \leq\left[u_{R_{A}}^{(n-m)}(x)-u_{R_{A}}^{(n)}(x)\right]-\left[u_{h_{A}}^{(n-m)}(x)-u_{h_{A}}^{(n)}(x)\right] \\
& -\frac{m}{n} u_{R_{A}}^{(n-m)}(x)+\frac{m}{n} u_{h_{A}}^{(n-m)}(x)+\frac{1}{n} \log \left\|V_{A}^{+}\left(T^{n} x\right)\right\|+\frac{\mathcal{C}_{A}}{n} .
\end{aligned}
$$

Using the triangle inequality, the two estimates on $r_{n}(x)$ that we have to establish follow from similar estimates on the upper and the lower bound functions above. These in turn follow from similar estimates on each of the terms between brackets as well as on the remaining terms.

First we prove separately that given a cocycle $R \in C_{r}^{\omega}\left(\mathbb{T}^{d}, \operatorname{Mat}_{k}(\mathbb{R})\right)$ of any dimension $k \geq 1$, if $f_{R}(x):=\operatorname{det}[R(x)] \not \equiv 0$, then for phases $x$ off of a small set, the following hold: the function $\left|u_{R}^{(n)}(x)\right|$ has a sublinear growth in $n$; the functions $u_{R}^{(n)}(x)$ and $u_{R}^{(n-1)}(x)$ corresponding to consecutive iterates of $R$, differ only slightly. We then apply these facts to the $k$-dimensional cocycle $R_{A}$ and to the one-dimensional cocycle $h_{A}$.

Since $R$ is a non-identically singular analytic cocycle, Proposition 6.3 in [14] is applicable, and it says that the functions $u_{R}^{(n)}(x)$ are uniformly bounded from above, and their failure to be bounded from below is captured by Birkhoff averages of the function $\log \left|f_{R}\right|$.

More precisely, there is $C=C(R)<\infty$ such that for all $x \in \mathbb{T}^{d}$,

$$
-C+\frac{1}{n} \sum_{i=0}^{n-1} \log \left|f_{R}\left(T^{i} x\right)\right| \leq u_{R}^{(n)}(x) \leq C .
$$

Furthermore, given that $f_{R}$ is analytic and non-identically zero, the function $\log \left|f_{R}\right| \in L^{p}\left(\mathbb{T}^{d}\right)$ for all $1 \leq p<\infty$. Then 2.29) implies uniform bounds in $n$ for the $L^{p}$-norms of $u_{R}^{(n)}$, so we may assume that

$$
\left\|u_{R}^{(n)}\right\|_{L^{2}\left(\mathbb{T}^{d}\right)},\left\|u_{R}^{(n)}\right\|_{L^{4}\left(\mathbb{T}^{d}\right)} \leq C .
$$


Applying the Łojasiewicz inequality to $f_{R}$, for some constants $S=$ $S(R)<\infty, \mathcal{C}_{0}=\mathcal{C}_{0}(R)>0$ and for any given $a \in(0,1)$ we have $\left|\left\{x: \log \left|f_{R}(x)\right| \leq-\mathcal{C}_{0} n^{1-a / 2}\right\}\right|=\left|\left\{x:\left|f_{R}(x)\right| \leq e^{-\mathcal{C}_{0} n^{1-a / 2}}\right\}\right| \leq S e^{-n^{1-a / 2}}$.

Then if $x$ is outside a set of measure $\leq n S e^{-n^{1-a / 2}}<S e^{-n^{1-a}}$,

$$
\frac{1}{n} \sum_{i=0}^{n-1} \log \left|f_{R}\left(T^{i} x\right)\right| \geq-\bigodot_{0} n^{1-a / 2},
$$

hence using (2.29), for these phases $x$,

$$
\left|u_{R}^{(n)}(x)\right| \lesssim \mathcal{C}_{0} n^{1-a / 2}
$$

Since

$$
\left\|R^{(n)}(x)\right\|=\left\|R\left(T^{n-1} x\right) R^{(n-1)}(x)\right\| \leq\|R\|_{L^{\infty}}\left\|R^{(n-1)}(x)\right\|,
$$

it follows that

$$
u_{R}^{(n)}(x)-u_{R}^{(n-1)}(x) \leq \frac{\log \|R\|_{L^{\infty}}}{n}-\frac{1}{n} u_{R}^{(n-1)}(x),
$$

hence for $x$ off of that sub-exponentially small set we have

$$
u_{R}^{(n)}(x)-u_{R}^{(n-1)}(x) \leq \frac{\mathcal{C}_{0}}{n}+\frac{\mathcal{C}_{0} n^{1-a / 2}}{n} \lesssim \mathcal{C}_{0} n^{-a / 2} .
$$

We also have

$$
\begin{aligned}
\left\|R^{(n-1)}(x)\right\| & =\left\|R\left(T^{n-1} x\right)^{-1} R^{(n)}(x)\right\| \leq\left\|R\left(T^{n-1} x\right)^{-1}\right\|\left\|R^{(n)}(x)\right\| \\
& =\frac{\left\|\operatorname{adj} R\left(T^{n-1} x\right)\right\|}{\left|\operatorname{det} R\left(T^{n-1} x\right)\right|}\left\|R^{(n)}(x)\right\| \leq \frac{\|R\|_{L^{\infty}}^{k-1}}{\left|f_{R}(x)\right|}\left\|R^{(n)}(x)\right\| .
\end{aligned}
$$

Then for $x$ off of a sub-exponentially small set, and due to previous considerations, it follows that

$$
\begin{aligned}
u_{R}^{(n-1)}(x)-u_{R}^{(n)}(x) & \leq \frac{(k-1) \log \|R\|_{L^{\infty}}}{n-1}-\frac{\log \left|f_{R}(x)\right|}{n-1}+\frac{1}{n-1} u_{R}^{(n)}(x) \\
& \lesssim \frac{\log \|R\|_{L^{\infty}}}{n}+\frac{\mathcal{C}_{0} n^{1-a / 2}}{n}+\frac{\mathcal{C}_{0} n^{1-a / 2}}{n} \lesssim \mathcal{C}_{0} n^{-a / 2}
\end{aligned}
$$

We conclude that if $x$ is outside a set of measure $\lesssim S e^{-n^{1-a}}$, then

$$
\left|u_{R}^{(n)}(x)-u_{R}^{(n-1)}(x)\right|<\mathcal{C}_{0} n^{-a / 2} .
$$

Furthermore, combining 2.31 with the fact that $\left\|u_{R}^{(n)}\right\|_{L^{4}\left(\mathbb{T}^{d}\right)} \leq C$ and using Cauchy-Schwarz, we also obtain

$$
\left\|u_{R}^{(n)}-u_{R}^{(n-1)}\right\|_{L^{2}\left(\mathbb{T}^{d}\right)}<\mathcal{C}_{0} n^{-a / 2} .
$$


Using telescoping sums and the fact that $m \leq n^{a / 6}$, from 2.31 we get that off of a sub-exponentially small set,

$$
\left|u_{R}^{(n \pm m)}(x)-u_{R}^{(n)}(x)\right| \lesssim \mathcal{C}_{0} m n^{-a / 2} \lesssim \mathcal{C}_{0} n^{-a / 3},
$$

and similarly, from 2.32 we get

$$
\left\|u_{R}^{(n \pm m)}-u_{R}^{(n)}\right\|_{L^{2}\left(\mathbb{T}^{d}\right)} \lesssim \mathcal{C}_{0} n^{-a / 3} .
$$

Furthermore, 2.30 implies that off of a sub-exponentially small set,

$$
\frac{m}{n}\left|u_{R}^{(n \pm m)}(x)\right| \lesssim \mathcal{C}_{0} m n^{-a / 2} \lesssim \mathcal{C}_{0} n^{-a / 3},
$$

and of course,

$$
\frac{m}{n}\left\|u_{R}^{(n \pm m)}\right\|_{L^{2}\left(\mathbb{T}^{d}\right)} \leq \frac{n^{a / 6}}{n} C<\mathcal{C}_{0} n^{-a / 3} .
$$

Going back to the upper and lower bounds (2.28) on the remainder function $r_{n}(x)$, applying the above with $R=R_{A}$ and $R=h_{A}$ takes care of most of the terms.

The only terms left to consider are $\frac{1}{n} \log \left\|V_{A}^{+}(x)\right\|$ and $\frac{1}{n} \log \left\|V_{A}^{+}\left(T^{n} x\right)\right\|$. To estimate them, simply apply Corollary 2.12 with $T:=\mathcal{C}_{0} n^{1-a / 3}$. This completes the proof.

Theorem 2.1. Given a quasi-periodic cocycle $A \in C_{r}^{\omega}\left(\mathbb{T}^{d}, \operatorname{Mat}_{m}(\mathbb{R})\right)$ with $L_{1}(A)>-\infty$ and $\omega \in \mathrm{DC}_{\mathrm{t}}$, there are constants $a_{0}=a_{0}(d)>0$, $b_{0}=b_{0}(d)>0$ and $\underline{n_{0}}=\underline{n_{0}}(A, d, t) \in \mathbb{N}$ such that if $n \geq \underline{n_{0}}$ then

$$
\left|\left\{x \in \mathbb{T}^{d}:\left|\frac{1}{n} \log \left\|A^{(n)}(x)\right\|-L_{1}^{(n)}(A)\right|>n^{-a_{0}}\right\}\right|<e^{-n^{b_{0}}} .
$$

Proof. We apply Proposition 2.16. There are: a (maximal rank) cocycle $R_{A} \in C_{r}^{\omega}\left(\mathbb{T}^{d}, \operatorname{Mat}_{k}(\mathbb{R})\right)$ with $\operatorname{det}\left[R_{A}(x)\right] \not \equiv 0$, a function $h_{A} \in$ $C_{r}^{\omega}\left(\mathbb{T}^{d}, \mathbb{R}\right)$ with $h_{A}(x) \not \equiv 0$ and small remainder functions $r_{n}(x)$ (in the sense of estimates (i) and (ii)) such that for all phases $x \in \mathbb{T}^{d}$ and all iterates $n$ after a threshold,

$$
u_{A}^{(n)}(x)=u_{R_{A}}^{(n)}(x)-u_{h_{A}}^{(n)}(x)+r_{n}(x) .
$$

Since $R_{A} \in C_{r}^{\omega}\left(\mathbb{T}^{d}, \operatorname{Mat}_{k}(\mathbb{R})\right)$ and $\operatorname{det}\left[R_{A}\right] \not \equiv 0$, the LDT estimate for non-identically singular cocycles applies (see Theorem 6.6 in [14.

Moreover, since we may regard $h_{A}$ as a one-dimensional quasi-periodic cocycle, and since $h_{A} \not \equiv 0$, the same result also applies to $h_{A}$.

Applying the aforementioned LDT estimate to the non-identically singular cocycles $R_{A}$ and $h_{A}$, we conclude that there are constants $\mathrm{C}_{0}=$ 
$\mathcal{C}_{0}(A, r)<\infty, a_{0}=a_{0}(d)>0, b_{0}=b_{0}(d)>0$ and $n_{0}=n_{0}(A, t) \in \mathbb{N}$ such that if $n \geq \underline{n_{0}}$ then

$$
\begin{aligned}
& \left|\left\{x \in \mathbb{T}^{d}:\left|u_{R_{A}}^{(n)}(x)-\left\langle u_{R_{A}}^{(n)}\right\rangle\right|>\mathcal{C}_{0} n^{-a_{0}}\right\}\right|<e^{-n^{b_{0}}} \\
& \left|\left\{x \in \mathbb{T}^{d}:\left|u_{h_{A}}^{(n)}(x)-\left\langle u_{h_{A}}^{(n)}\right\rangle\right|>\mathcal{C}_{0} n^{-a_{0}}\right\}\right|<e^{-n^{b_{0}}} .
\end{aligned}
$$

Then for any phase $x$ outside of these two exceptional sets, by 2.34 we have:

$$
\begin{aligned}
\left|u_{A}^{(n)}(x)-\left\langle u_{A}^{(n)}\right\rangle\right| & \leq\left|u_{R_{A}}^{(n)}(x)-\left\langle u_{R_{A}}^{(n)}\right\rangle\right| \\
& +\left|u_{h_{A}}^{(n)}(x)-\left\langle u_{h_{A}}^{(n)}\right\rangle\right|+\left|r_{n}(x)-\left\langle r_{n}\right\rangle\right| \\
& \leq \mathcal{C}_{0} n^{-a_{0}}+\mathcal{C}_{0} n^{-a_{0}}+\left|r_{n}(x)\right|+\left|\left\langle r_{n}\right\rangle\right| .
\end{aligned}
$$

Using the estimates (i) and (ii) on the remainder $r_{n}$ in Theorem 2.16 , after further excluding another sub-exponentially small set of phases $x$, we conclude that

$$
\left|u_{A}^{(n)}(x)-\left\langle u_{A}^{(n)}\right\rangle\right| \lesssim \mathcal{C}_{0} n^{-a_{0}}+\mathcal{C}_{0} n^{-\left(1-b_{0}\right) / 3}<n^{-a_{0}^{\prime}},
$$

for some $a_{0}^{\prime}>0$ and provided that $n \geq \underline{n_{0}}(A, d)$. The theorem is now proven.

\section{ESTIMATES ON SEPARATELY SUBHARMONIC FUNCTIONS}

A function $u: \Omega \subset \mathbb{C}^{d} \rightarrow[-\infty, \infty)$ is called separately subharmonic if it is continuous and subharmonic in each variable. Given an analytic cocycle $A \in C_{r}^{\omega}\left(\mathbb{T}^{d}, \operatorname{Mat}_{m}(\mathbb{R})\right)$, the functions $u_{A}^{(n)}(z):=\frac{1}{n} \log \left\|A^{(n)}(z)\right\|$ associated to its iterates are subharmonic along any complex line intersected with the domain $\mathcal{A}_{r}^{d}$, so in particular they are separately subharmonic on $\mathcal{A}_{r}^{d}$.

In this section we establish some general estimates on separately subharmonic functions $u(z)$ defined in a neighborhood of the torus. We later apply these estimates to the functions $u_{A}^{(n)}(z)$. In all of our estimates we assume an upper bound on $u(z)$ throughout the whole domain, and a lower bound at some point on the torus.

More precisely, throughout this section, we are given a separately subharmonic function $u: \mathcal{A}_{r}^{d} \rightarrow[-\infty, \infty)$ such that for some constant $C<\infty$ we have:

(1) $u(z) \leq C$ for all $z \in \mathcal{A}_{r}^{d}$;

(2) $u\left(x_{0}\right) \geq-C$ for some $x_{0} \in \mathbb{T}^{d}$. 
All the constants in the estimates derived here will depend only on $C, r$ and $d$, and not on the given function $u$ per se. Moreover, since the width $r$ of the annulus $\mathcal{A}_{r}$ and the number $d$ of variables will be fixed throughout, the dependence of these estimates on $r$ and $d$ will eventually stop being emphasized.

The estimates obtained will refer to the function $u(x)$ (the restriction of $u(z)$ to the torus $\mathbb{T}^{d}$ ) and they will be of the following kinds: an $L^{2}$ bound, a boosting of a weak a-priori deviation from the mean, and a quantitative version of the Birkhoff ergodic theorem. These types of estimates were previously derived for bounded separately subharmonic functions (see [5, 17, 25]). They were also derived (see Section 6.2 in our monograph [14]) for unbounded separately subharmonic functions that satisfy some uniform bounds along every line parallel to a coordinate axis, for instance if a lower bound as in item (2) is available for some point on every such line. This requirement is crucial as for $d=1$ the argument relies on the Riesz representation theorem for subharmonic functions, while for $d>1$ (when the Riesz representation theorem is not available), these results are obtained by applying the one-variable estimates along lines parallel to the coordinate axes.

However, we (have to) assume the availability of item (2) only at one point on the torus (we may assume it on a larger set of points, yet not one that intersects every line parallel to a coordinate axis). Hence the aforementioned results are not immediately applicable.

The idea is then to horizontally truncate the function $u(z)$ from below, at a sufficiently low level. The truncation is still separately subharmonic, but also bounded, so the kinds of estimates we are interested in do apply. Moreover, as the next lemma shows in a quantitative way, a separately subharmonic function cannot be too small for too long, hence the function itself and its (low enough level) truncation differ only on a small set of inputs. Finally, we note that this approach does create asymptotically large constants in all estimates, however, they will be manageable when applied to the functions $u_{A}^{(n)}(z)$, as part of an inductive process.

Lemma 3.1. There are constants $\gamma=\gamma(d)>0$ and $K_{r, d}<\infty$ such that for all $T \geq K_{r, d} C^{2}$ we have

$$
\left|\left\{x \in \mathbb{T}^{d}: u(x)<-T\right\}\right| \leq e^{-T^{\gamma}} .
$$

Proof. This result (in a slightly different formulation) was already established in [18]. The formulation in [18] says: if $u$ satisfies the upper bound $u(z) \leq C$ for all $z \in \mathcal{A}_{r}^{d}$, then there are constants $C_{d}$ and $C_{r, d}$, 
such that if for some $T<\infty$ and $\delta \in(0,1)$ we have

$$
\left|\left\{x \in \mathbb{T}^{d}: u(x)<-T\right\}\right|>\delta
$$

then

$$
\sup \left\{u(x): x \in \mathbb{T}^{d}\right\} \leq C_{r, d} C-\frac{T}{C_{r, d} \log ^{d}\left(C_{d} / \delta\right)} .
$$

When $d=1$, this is a direct consequence of Cartan's estimate (see Section 11.2 in [26]) for logarithmic potentials and the Riesz representation theorem for subharmonic functions. When $d>1$ it follows from the one-variable result and an inductive argument on $d$, using Fubini.

Fix $0<\gamma<\frac{1}{2 d}$. If $\left|\left\{x \in \mathbb{T}^{d}: u(x)<-T\right\}\right|>C_{d} e^{-T^{\gamma}}$, then applying (3.2) with $\delta:=C_{d} e^{-T^{\gamma}}$, we conclude that

$$
\sup \left\{u(x): x \in \mathbb{T}^{d}\right\}<C_{r, d} C-\frac{1}{C_{r, d}} T^{1 / 2} \leq-C,
$$

provided $T$ is large enough (i.e. $\left.T \geq\left(C_{r, d}\left(C_{r, d}+1\right)\right)^{2} C^{2}=: K_{r, d} C^{2}\right)$, contradicting the assumption (2) that $u\left(x_{0}\right) \geq-C$ for some $x_{0} \in \mathbb{T}^{d}$.

Lemma 3.2. The function $u(x)$ is in $L^{2}$ :

$$
\|u\|_{L^{2}\left(\mathbb{T}^{d}\right)} \lesssim C^{2}
$$

with the underlying constant depending on $r$ and $d$.

Proof. This is an easy consequence of the previous lemma. Indeed,

$$
\begin{aligned}
\|u\|_{L^{2}\left(\mathbb{T}^{d}\right)}^{2} & =\int_{\mathbb{T}^{d}}|u(x)|^{2} d x=\int_{0}^{\infty}\left|\left\{x \in \mathbb{T}^{d}:|u(x)|^{2}>T\right\}\right| d T \\
& =\int_{0}^{C^{*}}\left|\left\{x:|u(x)|^{2}>T\right\}\right| d T+\int_{C^{*}}^{\infty}\left|\left\{x:|u(x)|^{2}>T\right\}\right| d T,
\end{aligned}
$$

where we choose $C^{*}:=\max \left\{C^{2},\left(K_{r, d} C^{2}\right)^{2}\right\}$.

Note that

$$
\int_{0}^{C^{*}}\left|\left\{x:|u(x)|^{2}>T\right\}\right| d T \leq C^{*} \lesssim C^{4} .
$$

We estimate the second integral using Lemma 3.1. Note that since $u(x) \leq C$ for all $x \in \mathbb{T}^{d}$, and since $\sqrt{C^{*}} \geq C$, if $|u(x)|^{2}>T$ and $T \geq C^{*}$ then we must have that $u(x)<-\sqrt{T}$. Hence

$$
\begin{aligned}
\int_{C^{*}}^{\infty}\left|\left\{x:|u(x)|^{2}>T\right\}\right| d T & =\int_{C^{*}}^{\infty}|\{x: u(x)<-\sqrt{T}\}| d T \\
& \leq \int_{0}^{\infty} e^{-\sqrt{T}^{\gamma}} d T \lesssim 1
\end{aligned}
$$

where the value of the last integral depends on $\gamma$, so on $d$ only. 
Lemma 3.3. There are some (explicit) constants $p=p(d)<\infty, \alpha=$ $\alpha(d)>0$ and $\beta=\beta(d)>0$, such that if the weak a-priori estimate

$$
\left|\left\{x \in \mathbb{T}^{d}:|u(x)-\langle u\rangle|>\epsilon_{0}\right\}\right|<\epsilon_{1}
$$

holds for some $\epsilon_{0}, \epsilon_{1}>0$ small enough, with $\epsilon_{1} \leq \epsilon_{0}^{p}$ and $\epsilon_{0} \lesssim C^{-2}$, then the following sharper deviation estimate also holds

$$
\left|\left\{x \in \mathbb{T}^{d}:|u(x)-\langle u\rangle|>\epsilon_{0}^{\alpha}\right\}\right|<e^{-\epsilon_{0}^{-\beta}} .
$$

Proof. Let

$$
\widetilde{u}(z):=\max \left\{u(z),-\epsilon_{0}^{-1}\right\} \quad \text { for all } z \in \mathcal{A}_{r}^{d} .
$$

Then $\widetilde{u}$ is separately subharmonic and $-\epsilon_{0}^{-1} \leq \widetilde{u}(z) \leq C<\epsilon_{0}^{-1}$, so

$$
|\widetilde{u}(z)| \leq \epsilon_{0}^{-1} \quad \text { for all } z \in \mathcal{A}_{r}^{d} .
$$

By Lemma 3.1, there is $\gamma=\gamma(d)>0$ such that if we denote

$$
\mathcal{D}:=\left\{x \in \mathbb{T}^{d}: u(x)<-\epsilon_{0}^{-1}\right\},
$$

and since $\epsilon_{0}^{-1} \gtrsim C^{-2}$, then $|\mathcal{D}|<e^{-\epsilon_{0}^{-\gamma}}$ and $u(x)=\widetilde{u}(x)$ for all $x \in \mathcal{D}^{\complement}$.

Note that by Lemma 3.2. $\|u\|_{L^{2}\left(\mathbb{T}^{d}\right)} \lesssim C^{2}<\epsilon_{0}^{-1}$ and clearly $\|\widetilde{u}\|_{L^{2}\left(\mathbb{T}^{d}\right)} \leq$ $\|\widetilde{u}\|_{L^{\infty}\left(\mathbb{T}^{d}\right)} \leq \epsilon_{0}^{-1}$. Then by Cauchy-Schwarz,

$$
\begin{aligned}
|\langle u\rangle-\langle\widetilde{u}\rangle| & \leq \int_{\mathbb{T}^{d}}|u(x)-\widetilde{u}(x)| d x=\int_{\mathcal{D}}|u(x)-\widetilde{u}(x)| d x \\
& \leq\left(\|u\|_{L^{2}\left(\mathbb{T}^{d}\right)}+\|\widetilde{u}\|_{L^{2}\left(\mathbb{T}^{d}\right)}\right) \cdot|\mathcal{D}|^{1 / 2} \lesssim \epsilon_{0}^{-1} \cdot e^{-\frac{1}{2} \epsilon_{0}^{-\gamma}}<\epsilon_{0},
\end{aligned}
$$

provided $\epsilon_{0}$ is small enough (depending on $\gamma$, hence on $d$ ).

Let $\mathcal{B}:=\left\{x \in \mathbb{T}^{d}:|u(x)-\langle u\rangle|>\epsilon_{0}\right\}$. Then if $x \notin \mathcal{B} \cup \mathcal{D}$ we have

$$
\begin{aligned}
|\widetilde{u}(x)-\langle\widetilde{u}\rangle| & \leq|\widetilde{u}(x)-u(x)|+|u(x)-\langle u\rangle|+|\langle u\rangle-\langle\widetilde{u}\rangle| \\
& \leq 0+\epsilon_{0}+\epsilon_{0}=2 \epsilon_{0} .
\end{aligned}
$$

We may of course assume that $\epsilon_{1}>e^{-\epsilon_{0}^{-\gamma}}$, otherwise there is nothing to prove. Then

$$
\left|\left\{x \in \mathbb{T}^{d}:|\widetilde{u}(x)-\langle\widetilde{u}\rangle|>2 \epsilon_{0}\right\}\right| \leq|\mathcal{B}|+|\mathcal{D}| \leq \epsilon_{1}+e^{-\epsilon_{0}^{-\gamma}} \leq 2 \epsilon_{1} .
$$

We conclude that $\widetilde{u}(z)$ is a bounded separately subharmonic function, with a (weak) a-priori deviation from the mean bound like (3.4). As mentioned earlier, using Lemma 4.12 in [5], (3.6), can be boosted to a stronger estimate. We refer below to our more explicit formulation of this boosting (see Lemma 6.10 in [14]).

When $d=2$ and if $\epsilon_{1} \leq \epsilon_{0}^{8}$, for an absolute constant $c>0$ we get

$$
\left|\left\{x \in \mathbb{T}^{2}:|\widetilde{u}(x)-\langle\widetilde{u}\rangle|>\left(2 \epsilon_{0}\right)^{1 / 4}\right\}\right|<e^{-c\left[\left(2 \epsilon_{0}\right)^{1 / 4}+\mathcal{S}\left(2 \epsilon_{1}\right)^{1 / 8}\left(2 \epsilon_{0}\right)^{-1 / 2}\right]^{-1}},
$$


where the constant $\mathcal{S}$ is a multiple (depending on $r$ ) of some uniform bounds on $u(z)$. Thus in our case $\mathcal{S} \lesssim \epsilon_{0}^{-1}$, and assuming $\epsilon_{1} \leq \epsilon_{0}^{14}$,

$$
e^{-c\left[\left(2 \epsilon_{0}\right)^{1 / 4}+\mathcal{S}\left(2 \epsilon_{1}\right)^{1 / 8}\left(2 \epsilon_{0}\right)^{-1 / 2}\right]^{-1}}<e^{-c \epsilon_{0}^{-1 / 4}}<e^{-\epsilon_{0}^{-1 / 5}} .
$$

We conclude that if $d=2$ then

$$
\left|\left\{x \in \mathbb{T}^{2}:|\widetilde{u}(x)-\langle\widetilde{u}\rangle|>\left(2 \epsilon_{0}\right)^{1 / 4}\right\}\right|<e^{-\epsilon_{0}^{-1 / 5}} .
$$

A similar argument works for any $d$, where $1 / 4$ is replaced by $1 / 2 d$ etc.

Therefore, the conclusion (3.5) holds for the truncation $\widetilde{u}$ : for some powers $p<\infty$ and $\alpha, \beta>0$, all depending only on $d$,

$$
\left|\left\{x \in \mathbb{T}^{d}:|\widetilde{u}(x)-\langle\widetilde{u}\rangle|>\epsilon_{0}^{\alpha}\right\}\right|<e^{-\epsilon_{0}^{-\beta}} .
$$

It follows that a similar estimate holds for the original function $u$.

Let $\mathcal{B}^{\sharp}:=\left\{x \in \mathbb{T}^{d}:|\widetilde{u}(x)-\langle\widetilde{u}\rangle|>\epsilon_{0}^{\alpha}\right\}$. Then

$$
\left|\mathcal{B}^{\sharp} \cup \mathcal{D}\right| \leq\left|\mathcal{B}^{\sharp}\right|+|\mathcal{D}| \leq e^{-\epsilon_{0}^{-\beta}}+e^{-\epsilon_{0}^{-\gamma}},
$$

and if $x \notin \mathcal{B}^{\sharp} \cup \mathcal{D}$, then

$$
\begin{aligned}
|u(x)-\langle u\rangle| & \leq|u(x)-\widetilde{u}(x)|+|\widetilde{u}(x)-\langle\widetilde{u}\rangle|+|\langle\widetilde{u}\rangle-\langle u\rangle| \\
& \leq 0+\epsilon_{0}^{\alpha}+\epsilon_{0} \lesssim \epsilon_{0}^{\alpha} .
\end{aligned}
$$

We are now ready to formulate and to prove a quantitative version of Birkhoff's ergodic theorem for separately subharmonic functions satisfying the bounds in items (1) and (2).

Theorem 3.1. Let $u: \mathcal{A}_{r}^{d} \rightarrow[-\infty, \infty)$ be a separately subharmonic function such that for some constant $C<\infty$ we have

(1) $u(z) \leq C$ for all $z \in \mathcal{A}_{r}^{d}$;

(2) $u\left(x_{0}\right) \geq-C$ for some $x_{0} \in \mathbb{T}^{d}$.

Let $\omega \in \mathrm{DC}_{t}$ be a translation vector and denote by $S_{n} u(x):=\sum_{j=0}^{n-1} u\left(T^{j} x\right)$ the corresponding Birkhoff sums of $u(x)$.

There are constants $a=a(d)>0, b=b(d)>0, k=k(r, d)<\infty$ and $C_{r}<\infty$ such that for all $n \geq n_{0}:=k \cdot t^{-2}$ we have

$$
\left|\left\{x \in \mathbb{T}^{d}:\left|\frac{1}{n} S_{n} u(x)-\langle u\rangle\right|>\mathcal{S} n^{-a}\right\}\right|<e^{-n^{b}},
$$

where $\mathcal{S}=C_{r} C^{2}$.

Proof. We deduce this result from the corresponding one for bounded (a weaker assumption would suffice) separately subharmonic functions, by horizontal truncation. 
We refer to the more precise formulation of this result given by Theorem 6.5 in [14]: if $v(z)$ is separately subharmonic on $\mathcal{A}_{r}^{d}$ with some uniform bound $N$ (in particular, if $|v(z)| \leq N$ for all $z$ ), then for some constants $a, b>0$ depending on $d, C_{r}<\infty$ and for all $n \geq t^{-2}$, we have

$$
\left|\left\{x \in \mathbb{T}^{d}:\left|\frac{1}{n} S_{n} v(x)-\langle v\rangle\right|>C_{r} N n^{-a}\right\}\right|<e^{-n^{b}}
$$

Let $k=k(r, d)$ be large enough (so that for instance, $k^{a / 2} \geq K_{r, d}$, where $K_{r, d}$ is the constant from Lemma 3.1). Put $n_{0}:=k \cdot t^{-2}$.

Now fix (any) $n \geq n_{0}$ and define the truncation

$$
\widetilde{u}(z):=\max \left\{u(z),-C^{2} n^{a / 2}\right\} .
$$

Then $\widetilde{u}$ is separately subharmonic and bounded on $\mathcal{A}_{r}^{d}$, that is, $|\widetilde{u}(z)| \leq$ $C^{2} n^{a / 2}$ (as we may of course assume that $C \geq 1$ ). Hence (3.8) applies to $\widetilde{u}$ with $N=C^{2} n^{a / 2}$ and we have

$$
\left|\left\{x \in \mathbb{T}^{d}:\left|\frac{1}{n} S_{n} \widetilde{u}(x)-\langle\widetilde{u}\rangle\right|>\mathcal{S} n^{-a / 2}\right\}\right|<e^{-n^{b}} .
$$

On the other hand, if $\mathcal{D}:=\left\{x \in \mathbb{T}^{d}: u(x)<-C^{2} n^{a / 2}\right\}$, and since $C^{2} n^{a / 2} \geq K_{r, d} C^{2}$, then by Lemma 3.1 we have $|\mathcal{D}| \leq e^{-C^{2 \gamma} n^{a \gamma / 2}} \leq$ $e^{-n^{a \gamma / 2}}$. Moreover, if $x \notin \mathcal{D}$ then $u(x)=\widetilde{u}(x)$.

Let

$$
\mathcal{D}^{b}:=\bigcup_{j=0}^{n-1} T^{-j} \mathcal{D}
$$

Then clearly $\left|\mathcal{D}^{b}\right| \leq n e^{-n^{a \gamma / 2}}<e^{-n^{a \gamma / 4}}$, and if $x \notin \mathcal{D}^{b}$ then

$$
\frac{1}{n} S_{n} u(x)=\frac{1}{n} S_{n} \widetilde{u}(x) .
$$

Moreover, as before, by Lemma 3.2. $\|u\|_{L^{2}\left(\mathbb{T}^{d}\right)} \lesssim C^{2}$ and clearly $\|\widetilde{u}\|_{L^{2}\left(\mathbb{T}^{d}\right)} \leq\|\widetilde{u}\|_{L^{\infty}\left(\mathbb{T}^{d}\right)} \leq C^{2} n^{a / 2}$. Then by Cauchy-Schwarz,

$$
\begin{aligned}
|\langle u\rangle-\langle\widetilde{u}\rangle| & \leq \int_{\mathbb{T}^{d}}|u(x)-\widetilde{u}(x)| d x=\int_{\mathcal{D}}|u(x)-\widetilde{u}(x)| d x \\
& \leq\left(\|u\|_{L^{2}\left(\mathbb{T}^{d}\right)}+\|\widetilde{u}\|_{L^{2}\left(\mathbb{T}^{d}\right)}\right) \cdot|\mathcal{D}|^{1 / 2} \leq C^{2} n^{a / 2} e^{-\frac{1}{2} n^{a \gamma / 2}}<C^{2} e^{-n^{a \gamma / 4}} .
\end{aligned}
$$

Let $\mathcal{B}:=\left\{x \in \mathbb{T}^{d}:\left|\frac{1}{n} S_{n} \widetilde{u}(x)-\langle\widetilde{u}\rangle\right|>\mathcal{S} n^{-a / 2}\right\}$, so by (3.9) we have $|\mathcal{B}|<e^{-n^{b}}$. 
Then for any $x \notin \mathcal{D}^{b} \cup \mathcal{B}$, i.e. outside a sub-exponentially small set,

$$
\begin{aligned}
\left|\frac{1}{n} S_{n} u(x)-\langle u\rangle\right| & \leq\left|\frac{1}{n} S_{n} u(x)-\frac{1}{n} S_{n} \widetilde{u}(x)\right| \\
& +\left|\frac{1}{n} S_{n} \widetilde{u}(x)-\langle\widetilde{u}\rangle\right|+|\langle\widetilde{u}\rangle-\langle u\rangle| \\
& \leq 0+\mathcal{S} n^{-a / 2}+C^{2} e^{-n^{a \gamma / 4}}<\mathcal{S} n^{-a / 3},
\end{aligned}
$$

which completes the proof.

\section{The PRoof of The UNIFORM FIBER LDT}

In this section we present a new inductive procedure for establishing the uniform fiber LDT for quasi-periodic cocycles. This procedure might prove useful in other settings as well.

In Theorem 2.1 we established a non-uniform fiber LDT estimate for any given identically singular cocycle, by reduction to a maximal rank cocycle, for which this result was already available. We indicated in Section 2 that this reduction procedure is unstable under perturbations of the cocycles, and so it does not produce a uniform result, in the sense that the parameters in the LDT estimate may blow up as we perturb the cocycle. The idea is to use this non-uniform LDT just as an input to start the inductive process - by proximity to the given cocycle, we derive a uniform LDT at a fixed initial scale. Then we prove a fiber LDT estimate in the vicinity of the given cocycle, at larger and larger scales, using the avalanche principle. This inductive process allows us to keep track of the parameters in the LDT estimates and it ensures their uniformity.

Theorem 4.1. Given $A \in C_{r}^{\omega}\left(\mathbb{T}^{d}\right.$, $\left.\operatorname{Mat}_{m}(\mathbb{R})\right)$ with $L_{1}(A)>L_{2}(A)$ and $\omega \in \mathrm{DC}_{\mathrm{t}}$, there are constants $\delta=\delta(A)>0, n_{1}=n_{1}(A, t) \in \mathbb{N}$, $a_{1}=a_{1}(d)>0, b_{1}=b_{1}(d)>0$ so that if $\|B-A\|_{r} \leq \delta$ and $n \geq n_{1}$, then

$$
\left|\left\{x \in \mathbb{T}^{d}:\left|\frac{1}{n} \log \left\|B^{(n)}(x)\right\|-L_{1}^{(n)}(B)\right|>n^{-a_{1}}\right\}\right|<e^{-n^{b_{1}}} .
$$

Moreover, there is a constant $C=C(A)<\infty$ such that for all $n \geq 1$

$$
\left\|\frac{1}{n} \log \right\| B^{(n)}\|\|_{L^{2}\left(\mathbb{T}^{d}\right)}<C
$$

showing that $A$ is uniformly $L^{2}$-bounded.

Proof. We break down the argument into several steps. 
Before we begin. We define a threshold $n_{0}$ after which various estimates start being applicable. It is important to see that this threshold depends only on the given and fixed data: on the frequency $\omega$ (in fact, just on the parameter $t$ ) and on the cocycle $A$ (in fact just on $d, r$ and on some measurements of $A$ such as $\|A\|_{r}$ ).

For instance, if $n \geq n_{0}$, then the non-uniform fiber LDT estimate in Theorem 2.1, the quantitative Birkhoff Ergodic Theorem 3.1, as well as other results we use, are all applicable at scale $n$.

Asymptotically, any power of $n$ dominates a constant function and it is itself dominated by an exponential function of $n$. We assume $n_{0}$ to be large enough that any of these relations involving concrete size and measure bounds appearing throughout the proof will hold for $n \geq \underline{n_{0}}$.

As before, for any cocycle $B \in C_{r}^{\omega}\left(\mathbb{T}^{d}, \operatorname{Mat}_{m}(\mathbb{R})\right)$ and for any number of iterates $n \geq 1$, let $u_{B}^{(n)}: \mathcal{A}_{r}^{d} \rightarrow[-\infty, \infty)$ denote the separately subharmonic function

$$
u_{B}^{(n)}(z):=\frac{1}{n} \log \left\|B^{(n)}(z)\right\| .
$$

This function has the uniform upper bound $u_{B}^{(n)}(z) \leq \log \|B\|_{r}$ but of course, in general it is not bounded from below (which is the main point of this paper).

Recall the notation $\langle u\rangle$ for the mean of a function $u(x)$ on $\mathbb{T}^{d}$. In the case of the functions $u_{B}^{(n)}$ associated to iterates of a cocycle $B$, we also denote this mean by $L_{1}^{(n)}(B)$, and refer to it as the finite scale (maximal) Lyapunov exponent of $B$, since $L_{1}^{(n)}(B) \rightarrow L_{1}(B)$ as $n \rightarrow \infty$.

Let $\epsilon_{0}:=\frac{L_{1}(A)-L_{2}(A)}{50}>0$ (if $L_{2}(A)>-\infty$, otherwise just choose $\left.\epsilon_{0}:=1\right)$. Assume $\underline{n_{0}}$ to be large enough that for all $n, n^{\prime} \geq \underline{n_{0}}$ we have $\mid L_{1}^{(n)}(A)-L_{1}^{\left(n^{\prime}\right)}\left(\overline{A)} \mid<\epsilon_{0}\right.$.

Let $C_{0}$ be a finite constant such that $\log \|A\|_{r}<C_{0}$. This constant is chosen generously enough that if $B$ is a cocycle in a small, fixed neighborhood of $A$, then the bound $\log \|B\|_{r}<C_{0}$ also holds.

By Corollary 2.14, the functions $u_{A}^{(n)}$ are uniformly (in $\left.n\right) L^{2}$ bounded, hence we may assume that $\left\|u_{A}^{(n)}\right\|_{L^{2}\left(T^{d}\right)}<C_{0}$.

This of course also implies that for all $n \geq 1,\left|L_{1}^{(n)}(A)\right|<C_{0}$.

We will choose other, slightly larger constants $C_{0}<C_{1} \ll C_{2}$ that accommodate some extra polynomially small error terms, e.g. $C_{0}+$ ${\underline{n_{0}}}^{-p}<C_{1}$, for some power $p>0$ that depends on the given data, and similarly for the other constant.

For two real numbers $a$ and $b$, we write $a \asymp b$ or $a=\mathcal{O}(b)$ to mean $c_{1} a \leq b \leq c_{2} a$ for some absolute, positive constants $c_{1}$ and $c_{2}$. When 
it comes to (integer) scales, $m \asymp n$ is meant in a more strict way as $n \leq m \leq 3 n$.

The zeroth scale. We fix $n_{0} \geq n_{0}$ and define a neighborhood of $A$ such that the fiber LDT estimate on $A$ at any scale $m \asymp n_{0}$ transfers over to every cocycle $B$ in that neighborhood.

Let $\delta_{0}:=e^{-\bar{C} n_{0}}$ (where $\bar{C}$ will be chosen later) be the size of the neighborhood. From now on $B$ will be any cocycle with $\|B-A\|_{r}<\delta_{0}$.

For all scales $m \asymp n_{0}$ and for all $x \in \mathbb{T}^{d}$ we have

$$
\begin{aligned}
\|\| A^{(m)}(x)\|-\| B^{(m)}(x) \| \mid & \leq\left\|A^{(m)}(x)-B^{(m)}(x)\right\| \leq \delta_{0} m e^{C_{0} m} \\
& <e^{-\bar{C} n_{0}} e^{3\left(C_{0}+1\right) n_{0}}<e^{-2 C_{1} m},
\end{aligned}
$$

provided we choose $\bar{C} \gtrsim C_{0}+C_{1}$.

Since $\left|L_{1}^{(m)}(A)\right|<C_{0}$, at least for some $x_{0} \in \mathbb{T}^{d}$ we must have $\left|\frac{1}{m} \log \left\|A^{(m)}\left(x_{0}\right)\right\|\right|<C_{0}$, so

$$
\left\|A^{(m)}\left(x_{0}\right)\right\|>e^{-C_{0} m} .
$$

Combined with 4.3 this implies

$$
\left\|B^{(m)}\left(x_{0}\right)\right\|>e^{-C_{0} m}-e^{-2 C_{1} m} \gtrsim e^{-C_{0} m},
$$

hence

$$
u_{B}^{(m)}\left(x_{0}\right)=\frac{1}{m} \log \left\|B^{(m)}\left(x_{0}\right)\right\| \geq-C_{0}-\mathcal{O}\left(\frac{1}{m}\right)>-C_{1} .
$$

Combining this with the upper bound $u_{B}^{(m)}(z) \leq C_{0}$, Lemma 3.2 implies

$$
\left\|u_{B}^{(m)}\right\|_{L^{2}\left(T^{d}\right)} \lesssim C_{1}^{2}<C_{2} .
$$

Denote by $\mathcal{B}_{n}(A):=\left\{x \in \mathbb{T}^{d}:\left|\frac{1}{n} \log \left\|A^{(n)}(x)\right\|-L_{1}^{(n)}(A)\right|>n^{-a_{0}}\right\}$. If $n \geq \underline{n_{0}}$, then by Theorem 2.1. $\left|\mathcal{B}_{n}(A)\right| \leq e^{-n^{b_{0}}}$.

We are now ready to derive the proximity of the functions $u_{B}^{(m)}(x)$ and $u_{A}^{(m)}(x)$ for many phases $x \in \mathbb{T}^{d}$, as well as that of their means.

If $x \notin \mathcal{B}_{n}(A)$, then

$$
\frac{1}{n} \log \left\|A^{(n)}(x)\right\| \geq L_{1}^{(n)}(A)-n^{-a_{0}}>-C_{0}-{\underline{n_{0}}}^{-a_{0}}>-C_{1},
$$

hence $\left\|A^{(n)}(x)\right\|>e^{-C_{1} n}$.

Then if $m \asymp n_{0}$ and if $x \notin \mathcal{B}_{m}(A)$, a similar lower bound holds also for $B^{(m)}(x)$ :

$$
\begin{aligned}
\left\|B^{(m)}(x)\right\| & \geq\left\|A^{(m)}(x)\right\|-\left\|A^{(m)}(x)-B^{(m)}(x)\right\| \\
& >e^{-C_{1} m}-e^{-2 C_{1} m} \gtrsim e^{-C_{1} m},
\end{aligned}
$$


For $m \asymp n_{0}$ and $x \notin \mathcal{B}_{m}(A)$ we then have

$$
\begin{aligned}
\left|\frac{1}{m} \log \left\|B^{(m)}(x)\right\|-\frac{1}{m} \log \left\|A^{(m)}(x)\right\|\right| & \leq \frac{1}{m} \frac{\|\| A^{(m)}(x)\|-\| B^{(m)}(x) \| \mid}{\min \left\{\left\|A^{(m)}(x)\right\|,\left\|B^{(m)}(x)\right\|\right\}} \\
& <\frac{e^{-2 C_{1} m}}{e^{-C_{1} m}}=e^{-C_{1} m} .
\end{aligned}
$$

Moreover, by the previous estimates and Cauchy-Schwarz,

$$
\begin{aligned}
& \left|L_{1}^{(m)}(B)-L_{1}^{(m)}(A)\right| \leq \int_{\mathbb{T}^{d}}\left|\frac{1}{m} \log \left\|B^{(m)}(x)\right\|-\frac{1}{m} \log \left\|A^{(m)}(x)\right\|\right| d x \\
& \quad=\int_{\left(\mathcal{B}_{m}(A)\right)^{\complement}}\left|u_{B}^{(m)}(x)-u_{A}^{(m)}(x)\right| d x+\int_{\mathcal{B}_{m}(A)}\left|u_{B}^{(m)}(x)-u_{A}^{(m)}(x)\right| d x \\
& \quad \leq e^{-C_{1} m}+\left(\left\|u_{B}^{(m)}\right\|_{L^{2}\left(T^{d}\right)}+\left\|u_{A}^{(m)}\right\|_{L^{2}\left(T^{d}\right)}\right) \cdot\left|\mathcal{B}_{m}(A)\right|^{1 / 2} \\
& \quad \lesssim e^{-C_{1} m}+C_{2} e^{-m^{b_{0} / 2}}<m^{-a_{0}}
\end{aligned}
$$

hence

$$
\left|L_{1}^{(m)}(B)-L_{1}^{(m)}(A)\right|<m^{-a_{0}}<\epsilon_{0} .
$$

We conclude that for all $m \asymp n_{0}$ and $x \notin \mathcal{B}_{m}(A)$ (the set where the fiber LDT for $A$ and the estimates above all hold) we have

$$
\begin{aligned}
\left|\frac{1}{m} \log \left\|B^{(m)}(x)\right\|-L_{1}^{(m)}(B)\right| & \leq e^{-C_{1} m}+m^{-a_{0}} \\
& +\left|\frac{1}{m} \log \left\|A^{(m)}(x)\right\|-L_{1}^{(m)}(A)\right| \\
& \leq 3 m^{-a_{0}},
\end{aligned}
$$

which proves the following uniform fiber LDT at initial scales $m \asymp n_{0}$ :

$$
\left|\left\{x \in \mathbb{T}^{d}:\left|\frac{1}{m} \log \left\|B^{(m)}(x)\right\|-L_{1}^{(m)}(B)\right|>3 m^{-a_{0}}\right\}\right|<e^{-m^{b_{0}}} .
$$

Let us denote by $\mathcal{B}_{m}(B)$ the exceptional set in 4.5 , so for $m \asymp n_{0}$, $\left|\mathcal{B}_{m}(B)\right|<e^{-m^{b_{0}}}$.

Summary of estimates at scales $\asymp n_{0}$. We use 4.4 to derive two more estimates. If $m \asymp n_{0}$ then

$$
\left|L_{1}^{(m)}(B)\right|<\epsilon_{0}+\left|L_{1}^{(m)}(A)\right|<\epsilon_{0}+C_{0}<C_{1},
$$


and if $m, m^{\prime} \asymp n_{0}$ then

$$
\begin{aligned}
\left|L_{1}^{(m)}(B)-L_{1}^{\left(m^{\prime}\right)}(B)\right| & \leq\left|L_{1}^{(m)}(B)-L_{1}^{(m)}(A)\right|+\left|L_{1}^{\left(m^{\prime}\right)}(B)-L_{1}^{\left(m^{\prime}\right)}(A)\right| \\
& +\left|L_{1}^{(m)}(A)-L_{1}^{\left(m^{\prime}\right)}(A)\right| \\
& \leq m^{-a_{0}}+m^{-a_{0}}+\epsilon_{0}<2 \epsilon_{0} .
\end{aligned}
$$

We now summarize the estimates at scales $\asymp n_{0}$ that are needed at the next scale. Let $m, m^{\prime} \asymp n_{0}$. Then for any cocycle $B$ with $\|B-A\|_{r} \leq \delta_{0}$ the following hold:

$$
\begin{aligned}
\left\|u_{B}^{(m)}\right\|_{L^{2}\left(\mathbb{T}^{d}\right)} & <C_{2} \\
\left|L_{1}^{(m)}(B)\right| & <C_{1} \\
\left|L_{1}^{(m)}(B)-L_{1}^{\left(m^{\prime}\right)}(B)\right| & <2 \epsilon_{0} \\
\left|L_{1}^{(m)}(B)-L_{1}^{(m)}(A)\right| & <\epsilon_{0} \\
\left|\frac{1}{m} \log \right|\left|B^{(m)}(x) \|-L_{1}^{(m)}(B)\right| & \leq 3 m^{-a_{0}},
\end{aligned}
$$

where the last estimate holds for all $x \notin \mathcal{B}_{m}(B)$, with $\left|\mathcal{B}_{m}(B)\right|<e^{-m^{b_{0}}}$.

The first scale. Let the new scale $n_{1}$ be such that $n_{0}^{p^{\prime}} \leq n_{1} \leq e^{n_{0}^{b_{0} / 2}}$, where the power $p^{\prime}=p^{\prime}(d)$ is large enough but finite, and it will be made more explicit later. We fix (any) such integer $n_{1}$ and prove a uniform LDT for scales $\asymp n_{1}$. The idea is to break down the long block (i.e. product of matrices) $B^{\left(n_{1}\right)}(x)$ into blocks of length $\asymp n_{0}$ and apply the avalanche principle to these shorter blocks, thus relating certain quantities at scale $n_{1}$ to similar quantities at scales $\asymp n_{0}$.

Recalling a couple of relevant results. For the reader's convenience, we formulate below two crucial results proven in our monograph [14]: the avalanche principle (AP) and the uniform upper semicontinuity (u.s.c.) of the maximal Lyapunov exponent.

In [14] we proved a general version of the AP, one that applies to a sequence of higher dimensional, non-invertible matrices. Moreover, compared to previous versions of the principle, we also removed the constraint on the number of matrices in the sequence. Below we describe one of the statements in the AP that will be used in this proof. Recall the following terminology: if $g \in \operatorname{Mat}_{m}(\mathbb{R})$, let $s_{1}(g) \geq s_{2}(g) \geq \ldots \geq s_{m}(g) \geq 0$ be its singular values and let $\operatorname{gr}(g):=\frac{s_{1}(g)}{s_{2}(g)} \geq 1$ denote the ratio of its first two singular values. 
Proposition 4.1. There exists $c>0$ such that given $0<\epsilon<1$, $0<\kappa \leq c \epsilon^{2}$ and $g_{0}, g_{1}, \ldots, g_{n-1} \in \operatorname{Mat}_{m}(\mathbb{R})$, if

$$
\begin{aligned}
& \text { (gaps) } \operatorname{gr}\left(g_{i}\right)>\frac{1}{\kappa} \quad \text { for all } \quad 0 \leq i \leq n-1 \\
& \text { (angles) } \frac{\left\|g_{i} g_{i-1}\right\|}{\left\|g_{i}\right\|\left\|g_{i-1}\right\|}>\epsilon \quad \text { for all } \quad 1 \leq i \leq n-1
\end{aligned}
$$

then denoting $g^{(n)}:=g_{n-1} \ldots g_{1} g_{0}$, we have

$$
\left|\log \left\|g^{(n)}\right\|+\sum_{i=1}^{n-2} \log \left\|g_{i}\right\|-\sum_{i=1}^{n-1} \log \left\|g_{i} \cdot g_{i-1}\right\|\right| \lesssim n \cdot \frac{\kappa}{\epsilon^{2}} .
$$

We now describe the uniform u.s.c. of the maximal LE (see 24] for the original statement and, for a formulation that completely covers our setting, see Proposition 3.1 and Remark 3.2 in [14]).

Proposition 4.2. Let $A \in C_{r}^{\omega}\left(\mathbb{T}^{d}, \operatorname{Mat}_{m}(\mathbb{R})\right)$.

(i) Assume that $L_{1}(A)>-\infty$.

For every $\epsilon>0$, there are $\delta=\delta(A, \epsilon)>0$ and $n_{0}=n_{0}(A, \epsilon) \in \mathbb{N}$ such that if $B \in C_{r}^{\omega}\left(\mathbb{T}^{d}, \operatorname{Mat}_{m}(\mathbb{R})\right)$ with $\|B-A\|_{r}<\delta$ and if $n \geq n_{0}$ then for all $x \in \mathbb{T}^{d}$

$$
\frac{1}{n} \log \left\|B^{(n)}(x)\right\| \leq L_{1}(A)+\epsilon .
$$

(ii) Assume that $L_{1}(A)=-\infty$.

For every $L<\infty$, there are $\delta=\delta(A, L)>0$ and $n_{0}=n_{0}(A, L) \in \mathbb{N}$ such that if $B \in C_{r}^{\omega}\left(\mathbb{T}^{d}, \operatorname{Mat}_{m}(\mathbb{R})\right)$ with $\|B-A\|_{r}<\delta$ and if $n \geq n_{0}$, then for all $x \in \mathbb{T}^{d}$

$$
\frac{1}{n} \log \left\|B^{(n)}(x)\right\| \leq-L .
$$

We note that from Corollary 2.14 it follows that in our setting (of analytic, quasi-periodic cocycles), if $L_{1}(A)>-\infty$ then $A$ is automatically $L^{1}$-bounded. Hence all the assumptions of the more general corresponding result in [14] are satisfied.

Dividing into smaller blocks. Consider the block $B^{\left(n_{1}\right)}(x)$ of length $n_{1}$ and break it down into $n$ blocks, each having length $n_{0}$, except possibly for the last block, which will have length $m_{0} \asymp n_{0}$.

More precisely, let $n$ and $m_{0}$ be such that $n_{1}=(n-1) \cdot n_{0}+m_{0}$ and $n_{0} \leq m_{0}<2 m_{0}$. 
For $0 \leq i \leq n-2$ define

$$
\begin{aligned}
g_{i}=g_{i}(x) & :=B^{\left(n_{0}\right)}\left(T^{i n_{0}} x\right) \text { and } \\
g_{n-1}=g_{n-1}(x) & :=B^{\left(m_{0}\right)}\left(T^{(n-1) n_{0}} x\right) .
\end{aligned}
$$

Then $g^{(n)}=g_{n-1} \ldots g_{1} g_{0}=B^{\left(n_{1}\right)}(x)$.

We show that the geometrical assumptions "gaps" and "angles" of the AP are satisfied for these matrices if we choose the phases $x$ outside a certain small set.

The gaps condition. We establish this condition as a consequence of the uniform LDT (4.6e) and the estimate $(4.6 \mathrm{~d})$ proven for scales $m \asymp n_{0}$.

In the proof we use the uniform u.s.c. of the maximal LE in Proposition 4.2 and the hypothesis on the existence of a gap between the first two LE of $A$ (i.e. the fact that $L_{1}(A)-L_{2}(A)>0$ ).

Note that if $g \in \operatorname{Mat}_{m}(\mathbb{R})$, then

$$
\operatorname{gr}(g):=\frac{s_{1}(g)}{s_{2}(g)}=\frac{s_{1}^{2}(g)}{s_{1}(g) s_{2}(g)}=\frac{\|g\|^{2}}{\left\|\wedge_{2} g\right\|},
$$

where $\wedge_{2} g$ represents the second exterior power of $g$. If $m \asymp n_{0}$ then

$$
\frac{1}{m} \log \operatorname{gr}\left(B^{(m)}(x)\right)=2 \frac{1}{m} \log \left\|B^{(m)}(x)\right\|-\frac{1}{m} \log \left\|\wedge_{2} B^{(m)}(x)\right\| .
$$

We estimate each of the two terms above from below.

If $x \notin \mathcal{B}_{m}(B)$, the LDT estimate $4.6 \mathrm{e}$ implies

$$
u_{B}^{(m)}(x)=\frac{1}{m} \log \left\|B^{(m)}(x)\right\|>L_{1}^{(m)}(B)-3 m^{-a_{0}} .
$$

Combining this with the fact that when $m \asymp n_{0}$, by $\left.4.6 \mathrm{~d}\right), L_{1}^{(m)}(B)$ and $L_{1}^{(m)}(A)$ are close, we conclude that if $x \notin \mathcal{B}_{m}(B)$ then

$$
\frac{1}{m} \log \left\|B^{(m)}(x)\right\|>L_{1}^{(m)}(A)-\epsilon_{0}-3 m^{-a_{0}} \geq L_{1}(A)-2 \epsilon_{0} .
$$

To estimate $\frac{1}{m} \log \left\|\wedge_{2} B^{(m)}(x)\right\|$ from above, we apply the uniform u.s.c. of the maximal LE in Proposition 4.2, to the cocycle $\wedge_{2} B$. By making $\delta_{0}$ smaller, we may assume that $B$ is close enough to $A$ that in turn $\wedge_{2} B$ is close enough to $\wedge_{2} A$.

If $L_{1}\left(\wedge_{2} A\right)>-\infty$, which is equivalent to $L_{2}(A)>-\infty$, we have

$$
\frac{1}{n} \log \left\|\wedge_{2} B^{(n)}(x)\right\|<L_{1}\left(\wedge_{2} A\right)+\epsilon_{0}=L_{1}(A)+L_{2}(A)+\epsilon_{0}
$$

for all $n \geq \underline{n_{0}}$ and for all $x \in \mathbb{T}^{d}$ (which is the reason for calling this a uniform statement). Then for all $x \in \mathbb{T}^{d}$ we have

$$
-\frac{1}{m} \log \left\|\wedge_{2} B^{(m)}(x)\right\|>-L_{1}(A)-L_{2}(A)-\epsilon_{0} .
$$


Estimates (4.9), 4.11), (4.13) imply that if $m \asymp n_{0}$ and $x \notin \mathcal{B}_{m}(B)$, then

$$
\begin{aligned}
\frac{1}{m} \log \operatorname{gr}\left(B^{(m)}(x)\right) & >2 L_{1}(A)-4 \epsilon_{0}-L_{1}(A)-L_{2}(A)-\epsilon_{0} \\
& =L_{1}(A)-L_{2}(A)-5 \epsilon_{0}>19 \epsilon_{0},
\end{aligned}
$$

$\mathrm{SO}$

$$
\operatorname{gr}\left(B^{(m)}(x)\right) \geq e^{19 \epsilon_{0} m} \geq e^{19 \epsilon_{0} n_{0}}=: \frac{1}{\kappa_{n_{0}}} \text { for } x \notin \mathcal{B}_{m}(B) .
$$

The case when $L_{1}\left(\wedge_{2} A\right)=-\infty$ is treated similarly, the difference being that in 4.12 , instead of $L_{1}\left(\wedge_{2} A\right)+\epsilon_{0}$, we may take $-L$, with $L$ arbitrarily large (it will have to be chosen properly).

Estimate (4.14) shows that the gaps condition in the AP (Proposition 4.1) holds for $g=g(x):=B^{(m)}(x)$ provided $m \asymp n_{0}$ and $x \notin \mathcal{B}_{m}(B)$. Therefore, in order to hold for all matrices $g_{0}, g_{1}, \ldots, g_{n-1}$ defined above, we simply exclude the set of phases

$$
\mathcal{B}_{n_{0}}^{\text {gaps }}(B):=T^{-(n-1) n_{0}} \mathcal{B}_{m_{0}}(B) \cup \bigcup_{i=0}^{n-2} T^{-i n_{0}} \mathcal{B}_{n_{0}}(B)
$$

Note that $\left|\mathcal{B}_{n_{0}}^{\text {gaps }}(B)\right|<n e^{-n_{0}^{b_{0}}} \leq n_{1} e^{-n_{0}^{b_{0}}}<e^{-\frac{1}{2} n_{0}^{b_{0}}}$.

The angles condition. We derive this condition from the estimate 4.6c and the LDT estimate (4.6e), which are available at scales $m \asymp n_{0}$.

Let $m=n_{0}$ and $n_{0} \leq m^{\prime} \leq 2 n_{0}$, so that $m, m^{\prime}, m+m^{\prime} \asymp n_{0}$. Then the estimate $4.6 \mathrm{c}$ and the fiber LDT estimate (4.6e) apply at scales $m, m^{\prime}, m+m^{\prime}$, so if $x \notin \mathcal{B}_{m}(B) \cup T^{-m} \mathcal{B}_{m^{\prime}}(B) \cup \mathcal{B}_{m+m^{\prime}}(B)=: \mathcal{B}_{\left(m, m^{\prime}\right)}(B)$,

$$
\begin{aligned}
& \frac{\left\|B^{\left(m+m^{\prime}\right)}(x)\right\|}{\left\|B^{\left(m^{\prime}\right)}\left(T^{m} x\right)\right\|\left\|B^{(m)}(x)\right\|} \\
& >\frac{e^{\left(m+m^{\prime}\right)\left[L_{1}^{\left(m+m^{\prime}\right)}(B)-3\left(m+m^{\prime}\right)^{\left.-a_{0}\right]}\right.}}{e^{m^{\prime}\left[L_{1}^{\left(m^{\prime}\right)}(B)+3\left(m^{\prime}\right)^{-a_{0}}\right]} e^{m\left[L_{1}^{(m)}(B)+3 m^{-a_{0}}\right]}} \\
& >e^{m\left[L_{1}^{\left(m+m^{\prime}\right)}(B)-L_{1}^{(m)}(B)\right]+m^{\prime}\left[L_{1}^{\left(m^{\prime}+m\right)}(B)-L_{1}^{\left(m^{\prime}\right)}(B)\right]-\left(m+m^{\prime}\right) 9 n_{0}^{-a_{0}}} \\
& >e^{-\left(m+m^{\prime}\right)\left(2 \epsilon_{0}+9 n_{0}^{-a_{0}}\right)}>e^{-9 \epsilon_{0} n_{0}} .
\end{aligned}
$$

Therefore, if $x \notin \mathcal{B}_{\left(m, m^{\prime}\right)}(B)$, which is a set of measure $<3 e^{-n_{0}^{b}}$, we have

$$
\frac{\left\|B^{\left(m+m^{\prime}\right)}(x)\right\|}{\left\|B^{\left(m^{\prime}\right)}\left(T^{m} x\right)\right\|\left\|B^{(m)}(x)\right\|}>e^{-9 \epsilon_{0} n_{0}}=: \epsilon_{n_{0}} .
$$

Estimate 4.15) shows that the angles condition in the AP (Proposition 4.1) holds for $g=g(x):=B^{(m)}(x)$ and $g^{\prime}=g^{\prime}(x):=B^{\left(m^{\prime}\right)}\left(T^{m} x\right)$, 
provided the scales $m, m^{\prime}$ and the phases $x$ are as described. Therefore, in order to hold for all matrices $g_{0}, g_{1}, \ldots, g_{n-1}$ defined above, we simply exclude the set of phases

$$
\mathcal{B}_{n_{0}}^{\text {angles }}(B):=\mathcal{B}_{\left(n_{0}, m_{0}\right)}(B) \cup \bigcup_{i=0}^{n-2} T^{-i n_{0}} \mathcal{B}_{\left(n_{0}, n_{0}\right)}(B) .
$$

Note that $\left|\mathcal{B}_{n_{0}}^{\text {angles }}(B)\right|<3 n e^{-n_{0}^{b_{0}}}<e^{-\frac{1}{2} n_{0}^{b_{0}}}$.

Applying the avalanche principle. Let $\mathcal{B}_{n_{0}}^{\text {ap }}(B):=\mathcal{B}_{n_{0}}^{\text {gaps }}(B) \cup \mathcal{B}_{n_{0}}^{\text {angles }}(B)$, so $\left|\mathcal{B}_{n_{0}}^{\text {ap }}(B)\right|<e^{-\frac{1}{2} n_{0}^{b_{0}}}$.

Moreover, from 4.14, 4.15) we have $\frac{\kappa_{n_{0}}}{\epsilon_{n_{0}}^{2}}=e^{-\epsilon_{0} n_{0}} \ll 1$.

We conclude that if $x \notin \mathcal{B}_{n_{0}}^{\text {ap }}(B)$ then the AP in Proposition 4.1 applies and we have:

$$
\begin{aligned}
\log \| & B^{\left(n_{1}\right)}(x)\left\|=-\sum_{i=1}^{n-2} \log \right\| B^{\left(n_{0}\right)}\left(T^{i n_{0}} x\right) \| \\
& +\log \left\|B^{\left(n_{0}+m_{0}\right)}\left(T^{(n-2) n_{0}} x\right)\right\|+\sum_{i=1}^{n-2} \log \left\|B^{\left(2 n_{0}\right)}\left(T^{i n_{0}} x\right)\right\|+\mathcal{O}\left(n \frac{\kappa_{n_{0}}}{\epsilon_{n_{0}}^{2}}\right) .
\end{aligned}
$$

Divide both sides by $n_{1}$ and re-write the expression as

$$
\begin{aligned}
\frac{1}{n_{1}} \log \left\|B^{\left(n_{1}\right)}(x)\right\|= & -\frac{(n-2) n_{0}}{n_{1}} \frac{1}{n-2} \sum_{i=1}^{n-2} \frac{1}{n_{0}} \log \left\|B^{\left(n_{0}\right)}\left(T^{i n_{0}} x\right)\right\| \\
& +\frac{2(n-2) n_{0}}{n_{1}} \frac{1}{n-2} \sum_{i=1}^{n-2} \frac{1}{2 n_{0}} \log \left\|B^{\left(2 n_{0}\right)}\left(T^{i n_{0}} x\right)\right\| \\
& +\frac{n_{0}+m_{0}}{n_{1}} \frac{1}{n_{0}+m_{0}} \log \left\|B^{\left(n_{0}+m_{0}\right)}\left(T^{(n-2) n_{0}} x\right)\right\| \\
& +\mathcal{O}\left(\frac{n}{n_{1}} \frac{\kappa_{n_{0}}}{\epsilon_{n_{0}}^{2}}\right) .
\end{aligned}
$$

We estimate each of the terms above.

First off, since $n_{1} \asymp n n_{0}$, we have

$$
\frac{(n-2) n_{0}}{n_{1}}=1+\mathcal{O}\left(\frac{n_{0}}{n_{1}}\right), \frac{2(n-2) n_{0}}{n_{1}}=2+\mathcal{O}\left(\frac{n_{0}}{n_{1}}\right), \frac{n_{0}+m_{0}}{n_{1}}=\mathcal{O}\left(\frac{n_{0}}{n_{1}}\right) .
$$

Note that

$$
\frac{1}{n-2} \sum_{i=1}^{n-2} \frac{1}{n_{0}} \log \left\|B^{\left(n_{0}\right)}\left(T^{i n_{0}} x\right)\right\|=\frac{1}{n-2} \sum_{i=1}^{n-2} u_{B}^{\left(n_{0}\right)}\left(x+i n_{0} \omega\right)
$$

is a Birkhoff average of $u_{B}^{\left(n_{0}\right)}(x)$ over the translation vector $\omega_{0}:=n_{0} \omega$. 
The goal is to apply the quantitative Birkhoff Ergodic Theorem 3.1 to the separately subharmonic function $u=u_{B}^{\left(n_{0}\right)}$ and to the translation vector $\omega_{0}$. We need to verify the assumptions of that theorem and estimate the relevant parameters.

Recall that we always have $u_{B}^{\left(n_{0}\right)}(z) \leq C_{0}$ for all $z \in \mathcal{A}_{r}^{d}$. Moreover, by $4.6 \mathrm{~b}$ we also have $u_{B}^{\left(n_{0}\right)}(x)>-C_{1}$ for some $x \in \mathbb{T}^{d}$. Therefore, items (1) and (2) in Theorem 3.1 hold.

Since $\omega \in \mathrm{DC}_{t}$, for all $k \in \mathbb{Z}^{d} \backslash\{0\}$,

$$
\left\|k \cdot \omega_{0}\right\|=\left\|k \cdot n_{0} \omega\right\|=\left\|n_{0} k \cdot \omega\right\| \geq \frac{t}{\left|n_{0} k\right|^{d+1}}=\frac{t n_{0}^{-(d+1)}}{|k|^{d+1}},
$$

so $\omega_{0} \in \mathrm{DC}_{t_{0}}$, where $t_{0}:=t n_{0}^{-(d+1)}$.

Then Theorem 3.1 is applicable provided $n \geq k t_{0}^{-2}=k t^{-2} n_{0}^{2(d+1)}$, where $k=k(r, d)<\infty$. But $n \asymp \frac{n_{1}}{n_{0}}$ and $n_{1} \geq n_{0}^{p^{\prime}}$, hence for everything to work out we just have to choose $p^{\prime}>2(d+1)+1$ and $\underline{n_{0}}$ large enough depending on $t, r, d$.

There are positive constants $a=a(d), b=b(d)$ and $C_{r}<\infty$ such that

$$
\frac{1}{n-2} \sum_{i=1}^{n-2} u_{B}^{\left(n_{0}\right)}\left(x+i n_{0} \omega\right)=\left\langle u_{B}^{\left(n_{0}\right)}\right\rangle+\mathcal{O}\left(C_{r} C_{1}^{2} n^{-a}\right)
$$

provided $x$ is outside a set $\mathcal{B}_{1}$ with $\left|\mathcal{B}_{1}\right|<e^{-n^{b}}$.

We conclude that if $x \notin \mathcal{B}_{1}$, then

$$
\frac{1}{n-2} \sum_{i=1}^{n-2} \frac{1}{n_{0}} \log \left\|B^{\left(n_{0}\right)}\left(T^{i n_{0}} x\right)\right\|=L_{1}^{\left(n_{0}\right)}(B)+\mathcal{O}\left(C_{r} C_{1}^{2} n^{-a}\right) .
$$

Similarly, since all of this applies in fact to $u_{B}^{(m)}(x)$ for all $m \asymp n_{0}$, so in particular it applies to $m=2 n_{0}$, there is a set $\mathcal{B}_{2}$ with $\left|\mathcal{B}_{2}\right|<e^{-n^{b}}$ such that if $x \notin \mathcal{B}_{2}$ then

$$
\frac{1}{n-2} \sum_{i=1}^{n-2} \frac{1}{2 n_{0}} \log \left\|B^{\left(2 n_{0}\right)}\left(T^{i n_{0}} x\right)\right\|=L_{1}^{\left(2 n_{0}\right)}(B)+\mathcal{O}\left(C_{r} C_{1}^{2} n^{-a}\right) .
$$

Note that $C_{r} C_{1}^{2} n^{-a} \leq C_{r} C_{2} n_{0}^{a} n_{1}^{-a}<n_{1}^{-a / 2}$.

Since $n_{0}+m_{0} \asymp n_{0}$, we may apply 4.6e to $\frac{1}{n_{0}+m_{0}} \log \left\|B^{\left(n_{0}+m_{0}\right)}\right\|$. Hence there is a set $\mathcal{B}_{3}$ with $\left|\mathcal{B}_{3}\right|<e^{-n_{0}^{b_{0}}}$ such that if $x \notin \mathcal{B}_{3}$ then

$$
\left|\frac{1}{n_{0}+m_{0}} \log \left\|B^{\left(n_{0}+m_{0}\right)}\left(T^{(n-2) n_{0}} x\right)\right\|-L_{1}^{\left(n_{0}+m_{0}\right)}(B)\right| \leq 3 n_{0}^{-a_{0}} .
$$


Moreover, by $4.6 \mathrm{~b},\left|L_{1}^{\left(n_{0}+m_{0}\right)}(B)\right|<C_{1}$, hence for $x \notin \mathcal{B}_{3}$

$$
\left|\frac{1}{n_{0}+m_{0}} \log \left\|B^{\left(n_{0}+m_{0}\right)}\left(T^{(n-2) n_{0}} x\right)\right\|\right|<C_{1}+3 n_{0}^{-a_{0}}<C_{2} .
$$

Let $\mathcal{B}:=\mathcal{B}_{1} \cup \mathcal{B}_{2} \cup \mathcal{B}_{3}$. Note that since $n \asymp \frac{n_{1}}{n_{0}} \gg n_{0}$ and $n_{1} \leq e^{n_{0}^{b_{0} / 2}}$, we have

$$
|\mathcal{B}|<e^{-n^{b}}+e^{-n^{b}}+e^{-n_{0}^{b_{0}}}<n_{1}^{-p}
$$

where $p=p(d)$ is chosen large enough.

If $x \notin \mathcal{B}$, then putting it all together we have

$$
\begin{aligned}
\frac{1}{n_{1}} \log \left\|B^{\left(n_{1}\right)}(x)\right\|= & -\left[1+\mathcal{O}\left(\frac{n_{0}}{n_{1}}\right)\right]\left[L_{1}^{\left(n_{0}\right)}(B)+\mathcal{O}\left(n_{1}^{-a / 2}\right)\right] \\
& +\left[2+\mathcal{O}\left(\frac{n_{0}}{n_{1}}\right)\right]\left[L_{1}^{\left(2 n_{0}\right)}(B)+\mathcal{O}\left(n_{1}^{-a / 2}\right)\right] \\
& +\mathcal{O}\left(C_{2} \frac{n_{0}}{n_{1}}\right)+\mathcal{O}\left(e^{-\epsilon_{0} n_{0}}\right) \\
& =-L_{1}^{\left(n_{0}\right)}(B)+2 L_{1}^{\left(2 n_{0}\right)}(B)+\mathcal{O}\left(n_{1}^{-a / 2}\right) .
\end{aligned}
$$

We have shown that if $x \notin \mathcal{B}$ then

$$
u_{B}^{\left(n_{1}\right)}(x)=-L_{1}^{\left(n_{0}\right)}(B)+2 L_{1}^{\left(2 n_{0}\right)}(B)+\mathcal{O}\left(n_{1}^{-a / 2}\right) .
$$

Note that by $4.6 \mathrm{~b}$ and $4.6 \mathrm{c}$

$$
\begin{aligned}
-L_{1}^{\left(n_{0}\right)}(B)+2 L_{1}^{\left(2 n_{0}\right)}(B) & =L_{1}^{\left(n_{0}\right)}(B)-2\left[L_{1}^{\left(n_{0}\right)}(B)-L_{1}^{\left(2 n_{0}\right)}(B)\right] \\
& \geq-C_{1}-4 \epsilon_{0}
\end{aligned}
$$

so if $x \notin \mathcal{B}$, then by 4.16

$$
u_{B}^{\left(n_{1}\right)}(x)>-C_{1}-4 \epsilon_{0}-\mathcal{O}\left(n_{1}^{-a / 2}\right)>-2 C_{1} .
$$

Moreover, as always, $u_{B}^{\left(n_{1}\right)}(z) \leq C_{0}$ for all $z \in \mathcal{A}_{r}^{d}$.

Then by Lemma 3.2 we have

$$
\left\|u_{B}^{\left(n_{1}\right)}\right\|_{L^{2}\left(\mathbb{T}^{d}\right)} \lesssim C_{1}^{2}<C_{2} .
$$


We now show that $L_{1}^{\left(n_{1}\right)}(B) \approx-L_{1}^{\left(n_{0}\right)}(B)+2 L_{1}^{\left(2 n_{0}\right)}(B)$.

$$
\begin{aligned}
\mid L_{1}^{\left(n_{1}\right)}(B)+ & L_{1}^{\left(n_{0}\right)}(B)-2 L_{1}^{\left(2 n_{0}\right)}(B) \mid \\
& =\left|\int_{\mathbb{T}^{d}}\left[u_{B}^{\left(n_{1}\right)}(x)+L_{1}^{\left(n_{0}\right)}(B)-2 L_{1}^{\left(2 n_{0}\right)}(B)\right] d x\right| \\
& \leq \int_{\mathcal{B}^{\complement}}\left|u_{B}^{\left(n_{1}\right)}(x)+L_{1}^{\left(n_{0}\right)}(B)-2 L_{1}^{\left(2 n_{0}\right)}(B)\right| d x \\
& +\int_{\mathcal{B}}\left|u_{B}^{\left(n_{1}\right)}(x)+L_{1}^{\left(n_{0}\right)}(B)-2 L_{1}^{\left(2 n_{0}\right)}(B)\right| d x \\
& \lesssim n_{1}^{-a / 2}+C_{2}|\mathcal{B}|^{1 / 2} \lesssim n_{1}^{-a / 2}+C_{2} n_{1}^{-p / 2} \lesssim n_{1}^{-a / 2},
\end{aligned}
$$

where to estimate the integral on $\mathcal{B}$ we used (4.17) and Cauchy-Schwarz.

Hence

$$
\left|L_{1}^{\left(n_{1}\right)}(B)+L_{1}^{\left(n_{0}\right)}(B)-2 L_{1}^{\left(2 n_{0}\right)}(B)\right| \lesssim n_{1}^{-a / 2} .
$$

Combining 4.16) and (4.18) we obtain that if $x \notin \mathcal{B}$ then

$$
\left|u_{B}^{\left(n_{1}\right)}(x)-L_{1}^{\left(n_{1}\right)}(B)\right| \lesssim n_{1}^{-a / 2}<n_{1}^{-a / 3}
$$

Since $|\mathcal{B}|<n_{1}^{-p}$, where $p$ is large enough, we conclude that the separately subharmonic function $u_{B}^{\left(n_{1}\right)}$ satisfies the weak a-priori estimate

$$
\left|\left\{x \in \mathbb{T}^{d}:\left|u_{B}^{\left(n_{1}\right)}(x)-\left\langle u_{B}^{\left(n_{1}\right)}\right\rangle\right|>n_{1}^{-a / 3}\right\}\right|<n_{1}^{-p} .
$$

Lemma 3.3 is then applicable with $u=u_{B}^{\left(n_{1}\right)}, \epsilon_{0}=n_{1}^{-a / 3}, \epsilon_{1}=n_{1}^{-p}$, since items (1) and (2) also hold.

The weak a-priori estimate is then boosted to

$$
\left|\left\{x \in \mathbb{T}^{d}:\left|u_{B}^{\left(n_{1}\right)}(x)-\left\langle u_{B}^{\left(n_{1}\right)}\right\rangle\right|>n_{1}^{-\alpha a / 3}\right\}\right|<e^{-n_{1}^{\beta a / 3}}
$$

This establishes the uniform LDT estimate (4.1) at scale $n_{1}$ with parameters $a_{1}:=\alpha a / 3$ and $b_{1}:=\beta a / 3$, where $a, \alpha, \beta$ depend only on the number of variables $d$.

It is important to recall the provenance of these constants, namely the estimates in Theorem 3.1 and Lemma 3.3 on general separately subharmonic functions. Therefore, these constants will not change as we continue this process inductively.

Summary of estimates at scales $\asymp n_{1}$. Let us note that everything we have done at scale $n_{1}$ applies identically for any scale $m \asymp n_{1}$.

We now summarize the estimates at scales $\asymp n_{1}$ that are needed at the next scale. Let $m, m^{\prime} \asymp n_{1}$. Then for any cocycle $B$ with 
$\|B-A\|_{r} \leq \delta_{0}$ the following hold:

$$
\begin{aligned}
\left\|u_{B}^{(m)}\right\|_{L^{2}\left(\mathbb{T}^{d}\right)} & <C_{2} \\
\left|L_{1}^{(m)}(B)\right| & <C_{1} \\
\left|L_{1}^{(m)}(B)-L_{1}^{\left(m^{\prime}\right)}(B)\right| & <n_{1}^{-a / 3} \\
\left|L_{1}^{(m)}(B)-L_{1}^{(m)}(A)\right| & <9 \epsilon_{0}+n_{1}^{-a / 3}<10 \epsilon_{0} \\
\left|\frac{1}{m} \log \left\|B^{(m)}(x)\right\|-L_{1}^{(m)}(B)\right| & \leq m^{-a_{1}},
\end{aligned}
$$

where the last estimate holds for all $x \notin \mathcal{B}_{m}(B)$, with $\left|\mathcal{B}_{m}(B)\right|<e^{-m^{b_{1}}}$.

Let us explain why these estimates do indeed hold. First off, 4.19a corresponds to 4.17), while 4.19e is the uniform LDT just proven above for the scale $n_{1}$, and so for all other similar scales.

Moreover, 4.18 also holds for all $m \asymp n_{1}$ and we have

$$
\left|L_{1}^{(m)}(B)+L_{1}^{\left(n_{0}\right)}(B)-2 L_{1}^{\left(2 n_{0}\right)}(B)\right| \lesssim n_{1}^{-a / 2},
$$

which implies $\left|L_{1}^{(m)}(B)-L_{1}^{\left(m^{\prime}\right)}(B)\right| \lesssim n_{1}^{-a / 2}<n_{1}^{-a / 3}$, justifying (4.19c).

We rewrite 4.20 as

$$
\left|L_{1}^{(m)}(B)-L_{1}^{\left(n_{0}\right)}(B)+2\left[L_{1}^{\left(n_{0}\right)}(B)-L_{1}^{\left(2 n_{0}\right)}(B)\right]\right| \lesssim n_{1}^{-a / 2}
$$

and apply it to both $B$ and $A$. Together with $4.6 \mathrm{~d}$ ) and $4.6 \mathrm{c}$ we get

$$
\begin{aligned}
&\left|L_{1}^{(m)}(B)-L_{1}^{(m)}(A)\right| \leq\left|L_{1}^{\left(n_{0}\right)}(B)-L_{1}^{\left(n_{0}\right)}(A)\right| \\
&+\left|L_{1}^{(m)}(B)-L_{1}^{\left(n_{0}\right)}(B)+2\left[L_{1}^{\left(n_{0}\right)}(B)-L_{1}^{\left(2 n_{0}\right)}(B)\right]\right| \\
&+\left|L_{1}^{(m)}(A)-L_{1}^{\left(n_{0}\right)}(A)+2\left[L_{1}^{\left(n_{0}\right)}(A)-L_{1}^{\left(2 n_{0}\right)}(A)\right]\right| \\
&+2\left|L_{1}^{\left(n_{0}\right)}(B)-L_{1}^{\left(2 n_{0}\right)}(B)\right|+2\left|L_{1}^{\left(n_{0}\right)}(A)-L_{1}^{\left(2 n_{0}\right)}(A)\right| \\
& \leq \epsilon_{0}+\mathcal{O}\left(n_{1}^{-a / 2}\right)+\mathcal{O}\left(n_{1}^{-a / 2}\right)+4 \epsilon_{0}+4 \epsilon_{0} \\
&<9 \epsilon_{0}+n_{1}^{-a / 3}<10 \epsilon_{0} .
\end{aligned}
$$

This justifies (4.19d), which then implies

$$
\left|L_{1}^{(m)}(B)\right|<10 \epsilon_{0}+\left|L_{1}^{(m)}(A)\right|<10 \epsilon_{0}+C_{0}<C_{1},
$$

proving $4.19 \mathrm{~b}$.

The next scales. We explain how the inductive procedure continues. The argument is identical to the one used to derive the estimates (4.19) at scales $\asymp n_{1}$ from the corresponding estimates (4.6) at scales $\asymp n_{0}$. 
For every scale $n_{1}$ in the range prescribed earlier, let the next scale $n_{2}$ be such that $n_{1}^{p^{\prime}} \leq n_{2} \leq e^{n_{1}^{b_{1} / 2}}$. Break down the block $B^{\left(n_{2}\right)}(x)$ into blocks of lengths $\asymp n_{1}$ and set out to apply the avalanche principe to the resulting chain of matrices.

The "gap" condition for blocks of lengths $\asymp n_{1}$ is ensured by the uniform LDT (4.19e) and the estimate (4.19d) proven for scales $m \asymp n_{1}$. The proof uses the uniform upper semicontinuity of the maximal LE in Proposition 4.2 (which holds for all scales) and the hypothesis on the existence of a gap between the first two LE of $A$. It proceeds as at the previous scale, the only difference being that from (4.19e), for $m \asymp n_{1}$, 4.10) becomes instead

$$
u_{B}^{(m)}(x)=\frac{1}{m} \log \left\|B^{(m)}(x)\right\|>L_{1}^{(m)}(B)-m^{-a_{1}} .
$$

Together with estimate $4.19 \mathrm{~d}$ ) (which compared to $4.6 \mathrm{~d}$ ) has the factor 10), this shows that the analogue of (4.11) is

$$
\frac{1}{m} \log \left\|B^{(m)}(x)\right\|>L_{1}^{(m)}(A)-10 \epsilon_{0}-m^{-a_{1}} \geq L_{1}(A)-11 \epsilon_{0} .
$$

Moreover, the "angles" condition for blocks of lengths $\asymp n_{1}$ is ensured by $4.19 \mathrm{e}$ and $4.19 \mathrm{c}$ ) exactly the same way they were derived at the previous scale. In fact, the bound obtained will be stronger, because 4.19e and 4.19c are sharper than their counterparts 4.6e and $4.6 \mathrm{c})$ at scale $\asymp n_{0}$.

After using the AP, the next step is to apply the quantitative Birkhoff Ergodic Theorem 3.1 to the separately subharmonic functions $u_{B}^{(m)}(z)$ with $m \asymp n_{1}$ and translation vector $\omega_{1}:=n_{1} \omega$. The assumptions in this theorem hold as follows. The upper bound $\sup _{z \in \mathcal{A}_{r}^{d}} u_{B}^{(m)}(z) \leq C_{0}$ is always true, ensuring that item (1) holds. Estimate $4.19 \mathrm{~b}$ implies $\left|\left\langle u_{B}^{(m)}\right\rangle\right|=\left|L_{1}^{(m)}(B)\right|<C_{1}$, hence for some $x_{0} \in \mathbb{T}^{d}, u_{B}^{(m)}\left(x_{0}\right)>-C_{1}$, showing that item (2) also holds. Finally, because $n_{2} \geq n_{1}^{p^{\prime}}$, the number $n \asymp \frac{n_{2}}{n_{1}}$ of iterates is large enough relative to the parameter defining the Diophantine condition satisfied by $\omega_{1}$.

The analogue of the bookkeeping in 4.19 for the next scale is derived in the same way, where the bounds in (4.19b), 4.19a) and 4.19e do not change, while the one in $4.19 \mathrm{c}$ becomes sharper as a result of the scale increase: $\left|L_{1}^{(m)}(B)-L_{1}^{\left(m^{\prime}\right)}(B)\right|<n_{2}^{-a / 3}$ for all $m, m^{\prime} \asymp n_{2}$.

We provide more details regarding the derivation of the analogue of $4.19 \mathrm{~d})$ at scale $n_{2}$, as this is the place where the estimate worsens slightly from scale to scale, although the additional errors form a 
summable series that does not exceed $10 \epsilon_{0}$. We have

$$
\begin{aligned}
\left|L_{1}^{(m)}(B)-L_{1}^{(m)}(A)\right| \leq\left|L_{1}^{\left(n_{1}\right)}(B)-L_{1}^{\left(n_{1}\right)}(A)\right| \\
+\left|L_{1}^{(m)}(B)-L_{1}^{\left(n_{1}\right)}(B)+2\left[L_{1}^{\left(n_{1}\right)}(B)-L_{1}^{\left(2 n_{1}\right)}(B)\right]\right| \\
+\left|L_{1}^{(m)}(A)-L_{1}^{\left(n_{1}\right)}(A)+2\left[L_{1}^{\left(n_{1}\right)}(A)-L_{1}^{\left(2 n_{1}\right)}(A)\right]\right| \\
+2\left|L_{1}^{\left(n_{1}\right)}(B)-L_{1}^{\left(2 n_{1}\right)}(B)\right|+2\left|L_{1}^{\left(n_{1}\right)}(A)-L_{1}^{\left(2 n_{1}\right)}(A)\right| \\
\leq\left(9 \epsilon_{0}+n_{1}^{-a / 3}\right)+\mathcal{O}\left(n_{2}^{-a / 2}\right)+\mathcal{O}\left(n_{2}^{-a / 2}\right)+2 n_{1}^{-a / 3}+2 n_{1}^{-a / 3} \\
=9 \epsilon_{0}+5 n_{1}^{-a / 3}+\mathcal{O}\left(n_{2}^{-a / 2}\right)<9 \epsilon_{0}+5 n_{1}^{-a / 3}+n_{2}^{-a / 3} \\
\\
<9 \epsilon_{0}+5 \sum_{k=1}^{\infty} n_{k}^{-a / 3}<9 \epsilon_{0}+10 n_{1}^{-a / 3}<10 \epsilon_{0} .
\end{aligned}
$$

The argument continues the same way with scales

$$
\ldots \gg n_{k} \gg \ldots \gg n_{3} \gg n_{2} \gg n_{1}
$$

chosen such that $n_{k}^{p^{\prime}} \leq n_{k+1} \leq e^{n_{k}^{b_{1} / 2}}$, hence their ranges overlap. Therefore, the uniform LDT (4.1) holds for all $n \geq n_{1}:=n_{0}^{p^{\prime}}$. Furthermore, the uniform $L^{2}$ bound $(4.2$ holds by $4.19 \mathrm{a}$ ) and its analogues at higher scales. Strictly speaking, we have derived it only for $n \geq n_{1}$. However, at the cost of decreasing slightly the size $\delta_{0}$ of the neighborhood around $A$, we may assume that 4.3 holds in fact for all $m \leq n_{0}^{p^{\prime}}$, hence the argument following this estimate ensures the uniform $L^{2}$ bound also at scales $m \leq \underline{n_{1}}$.

\section{The PROOFs OF THE MAIN STATEMENTS}

Large deviations type estimates for iterates of linear cocycles can be used to establish the continuity of the corresponding Lyapunov exponents. This was the subject of our monograph [14]. The crucial component of the continuity argument was the uniformity of the estimates in the cocycle.

Given any cocycle $A \in C_{r}^{\omega}\left(\mathbb{T}^{d}, \operatorname{Mat}_{m}(\mathbb{R})\right)$ with $L_{1}(A)>L_{2}(A)$, in Theorem 4.1 we proved uniform fiber LDT estimates in a neighborhood of $A$; moreover, we established that $A$ is uniformly $L^{2}$-bounded, i.e. the uniform estimate 4.2 on the iterates of any nearby cocycle.

The abstract continuity theorem (ACT) in Chapter 3 of this book contains additional assumptions. However, in our present setting of analytic, quasi-periodic cocycles - in fact for any space of cocycles over a uniquely ergodic base dynamics on a compact metric space - they are automatically satisfied (the reader may consult Section 6.4 in [14] for 
the complete argument in the non-identical singular case). Therefore, the ACT is applicable and it establishes Theorem 1.2, where the weakHölder modulus of continuity is a consequence of the sub-exponential rate of decay in the LDT (4.1).

The continuity of the Oseledets filtration and decomposition are similarly consequences of an abstract statement we derived in Chapter 4 of [14. The assumptions in this statement are the same as for the continuity of the Lyapunov exponents, hence Theorem 1.3 is also established.

Let us move on to the applications of the continuity theorem of the Lyapunov exponents for identically singular cocycles to the positivity and simplicity of the Lyapunov exponents.

We begin with the proof of Theorem 1.4 .

For every $\delta \in \mathbb{R}$ define the cocycle

$$
S_{\delta}:=\left[\begin{array}{cc}
M & \delta N \\
\delta P & \delta Q
\end{array}\right]
$$

Note that $S_{0}=\left[\begin{array}{cc}M & 0 \\ 0 & 0\end{array}\right]$ is identically singular.

We can write

$$
A_{\lambda}=\left[\begin{array}{cc}
\lambda M & N \\
P & Q
\end{array}\right]=\lambda\left[\begin{array}{cc}
M & \frac{1}{\lambda} N \\
\frac{1}{\lambda} P & \frac{1}{\lambda} Q
\end{array}\right]=\lambda S_{\frac{1}{\lambda}}
$$

so for every $1 \leq k \leq m, L_{k}\left(A_{\lambda}\right)=\log |\lambda|+L_{k}\left(S_{\frac{1}{\lambda}}\right)$.

$$
\text { As }|\lambda| \rightarrow \infty, S_{\frac{1}{\lambda}} \rightarrow S_{0}=\left[\begin{array}{cc}
M & 0 \\
0 & 0
\end{array}\right] \text {, and } L_{l}\left(S_{0}\right)=L_{l}(M)>-\infty
$$

(because $\operatorname{det}[M(x)] \not \equiv 0)$.

Then if $|\lambda|$ is large enough, by the continuity Theorem 1.2 we have

$$
L_{l}\left(A_{\lambda}\right)=\log |\lambda|+L_{l}\left(S_{\frac{1}{\lambda}}\right)>\log |\lambda|+L_{l}(M)-1,
$$

which establishes 1.7 a with $C_{0}:=-L_{l}(M)+1<\infty$.

The simplicity statement in item (b) of the theorem follows the same way. The $l$ largest Lyapunov exponents of $S_{0}$ are exactly the Lyapunov exponents of $M$, which are assumed to be simple. Then the quantitative part of Theorem 1.2 applies to each of these exponents, so there is a weak-Hölder modulus of continuity function $w(h)$ so that for $|\delta| \ll 1$ and for every $1 \leq k \leq l$ we have

$$
\left|L_{k}\left(S_{\delta}\right)-L_{k}(M)\right|=\left|L_{k}\left(S_{\delta}\right)-L_{k}\left(S_{0}\right)\right| \leq w(|\delta|) \rightarrow 0 \quad \text { as } \delta \rightarrow 0 .
$$


This then translates into

$$
\left|L_{k}\left(A_{\lambda}\right)-\log \right| \lambda\left|-L_{k}(M)\right| \leq w\left(\frac{1}{|\lambda|}\right),
$$

thus proving $1.7 \mathrm{~b}$.

The proof of Theorem 1.5 proceeds the same way as that of Proposition 1.1. We first factor out $\lambda$ to write

$$
A_{\lambda, E}=\lambda\left[\begin{array}{cc}
U(x)\left(F(x)+\frac{1}{\lambda} R(x)-\frac{E}{\lambda} I\right) & \frac{1}{\lambda} N \\
\frac{1}{\lambda} P & \frac{1}{\lambda} Q
\end{array}\right] .
$$

Make the change of coordinates $\delta=\frac{1}{\lambda}, s=\frac{E}{\lambda}$, and consider the cocycle

$$
S_{\delta, s}(x):=\left[\begin{array}{cc}
U(x)(F(x)+\delta R(x)-s I) & \delta N \\
\delta P & \delta Q
\end{array}\right],
$$

so we have $A_{\lambda, E}=\lambda S_{\frac{1}{\lambda}, \frac{E}{\lambda}}$.

Then

$$
\begin{gathered}
L_{l}\left(A_{\lambda, E}\right)=\log |\lambda|+L_{l}\left(S_{\frac{1}{\lambda}, \frac{E}{\lambda}}\right) . \\
\text { For every } s \in \mathbb{R}, S_{0, s}(x)=\left[\begin{array}{cc}
U(x)(F(x)-s I) & 0 \\
0 & 0
\end{array}\right] \text {, so } \\
L_{l}\left(S_{0, s}\right)=L_{l}(U(x)(F(x)-s I))>-\infty
\end{gathered}
$$

because by our assumptions, $\operatorname{det}[U(x)(F(x)-s I)] \not \equiv 0$.

By the continuity Theorem 1.2 , the map $(\delta, s) \mapsto L_{l}\left(S_{\delta, s}\right)$ is continuous. Therefore, locally near every point $(0, s)$, this map has a finite lower bound. By compactness, given any compact interval $I$, there are $\delta_{0}>0$ and $C_{0}<\infty$ such that on $\left[-\delta_{0}, \delta_{0}\right] \times I$, the map $(\delta, s) \mapsto L_{l}\left(S_{\delta, s}\right)$ is bounded from below by $-C_{0}$.

Translating this back, it follows that for $|\lambda|$ large enough, if $\frac{E}{\lambda}$ is bounded, say $|E| \leq 2|\lambda|\|F\|_{r}$, then

$$
L_{l}\left(A_{\lambda, E}\right)>\log |\lambda|-C_{0} .
$$

Next we factor out $E$, to get

$$
A_{\lambda, E}=E\left[\begin{array}{cc}
U(x)\left(\frac{\lambda}{E} F(x)+\frac{1}{E} R-I\right) & \frac{1}{E} N \\
\frac{1}{E} P & \frac{1}{E} Q
\end{array}\right]
$$

and make the change of variables $\delta=\frac{1}{E}, s=\frac{\lambda}{E}$. 
By the same continuity and compactness argument, we conclude that for $\frac{\lambda}{E}$ bounded, say $|\lambda| \leq 2|E|\|F\|_{r}$, and for $|E|$ large enough (which would happen if we chose $|\lambda|$ large enough), we have

$$
L_{l}\left(A_{\lambda, E}\right)>\log |E|-C_{0}^{\prime}>\log |\lambda|-C_{0},
$$

which completes the proof of the theorem.

\section{Consequences for Block JACOBI Operators}

In this section we present some immediate applications of our main statements to block Jacobi operators (also called strip or band lattice operators). These types of operators generalize the one-dimensional lattice Schrödinger operator described in Section 1. Our applications are concerned with the positivity, continuity and (local) simplicity of the Lyapunov exponents of the corresponding eigenvalue equation, and also with the continuity of the integrated density of states (IDS).

Let us begin by describing a block Jacobi operator.

Fix a translation vector $\omega \in \mathbb{T}^{d}$. Let $W, R, F \in C_{r}^{\omega}\left(\mathbb{T}^{d}, \operatorname{Mat}_{l}(\mathbb{R})\right)$. Assume that for all phases $x \in \mathbb{T}^{d}, R(x)$ and $F(x)$ are symmetric matrices, that $W$ is not identically singular and denote by $W^{T}(x)$ the transpose of the matrix $W(x)$. Moreover, for all $n \in \mathbb{N}$, denote

$$
W_{n}(x):=W(x+n \omega), R_{n}(x):=R(x+n \omega), F_{n}(x):=F(x+n \omega)
$$

A quasi-periodic block Jacobi operator is an operator $H=H_{\lambda}(x)$ acting on $l^{2}\left(\mathbb{Z}, \mathbb{R}^{l}\right)$ by

$\left[H_{\lambda}(x) \vec{\psi}_{n}:=-\left(W_{n+1}(x) \vec{\psi}_{n+1}+W_{n}^{T}(x) \vec{\psi}_{n-1}+R_{n}(x) \vec{\psi}_{n}\right)+\lambda F_{n}(x) \vec{\psi}_{n}\right.$

where $\vec{\psi}=\left\{\vec{\psi}_{n}\right\}_{n \in \mathbb{Z}} \in l^{2}\left(\mathbb{Z}, \mathbb{R}^{l}\right)$ is any state, $x \in \mathbb{T}^{d}$ is a phase that introduces some randomness into the system and $\lambda \neq 0$ is a coupling constant.

This model contains all quasi-periodic, finite range hopping Schrödinger operators on integer or band integer lattices (which in some sense may be regarded as approximations of higher dimensional lattices). The hopping term is given by the "weighted" Laplacian:

$$
\left[\Delta_{W}(x) \vec{\psi}\right]_{n}:=-W_{n+1}(x) \vec{\psi}_{n+1}+W_{n}^{T}(x) \vec{\psi}_{n-1}+R_{n}(x) \vec{\psi}_{n}
$$

where the hopping amplitude is encoded by the quasi-periodic matrix valued functions $W_{n}(x)$ and $R_{n}(x)$.

The potential is given by the quasi-periodic matrix valued function $\lambda F_{n}(x)$. 
The more relevant situation from a physical point of view is when the potential function $F(x)$ is a diagonal matrix, while the entries of the weight $W(x)$ are trigonometric polynomials.

The associated Schrödinger equation

$$
H_{\lambda}(x) \vec{\psi}=E \vec{\psi}
$$

for a (generalized) state $\vec{\psi}=\left\{\vec{\psi}_{n}\right\}_{n \in \mathbb{Z}} \subset \mathbb{R}^{l}$ and energy $E \in \mathbb{R}$, gives rise to a cocycle $A_{\lambda, E}(x)$ of dimension $m=2 l$. Let $L_{k}(E)=L_{k}\left(A_{\lambda, E}\right)$ denote its $k$-th Lyapunov exponent. An easy calculation shows that

$A_{\lambda, E}(x)=\left[\begin{array}{cc}W^{-1}(x+\omega)(\lambda F(x)+R(x)-E I) & -W^{-1}(x+\omega) W^{T}(x) \\ I & 0\end{array}\right]$

and that this cocycle can be conjugated to a symplectic cocycle (see Section 8 in [12] for more details).

Note that since $W(x)$ is analytic and $\operatorname{det}[W(x)] \not \equiv 0, W^{-1}(x)$ exists almost everywhere, so the cocycle $A_{\lambda, E}(x)$ is defined almost everywhere.

Then the Lyapunov exponents are well defined and they satisfy the relations

$$
L_{1}(E) \geq \ldots \geq L_{l}(E) \geq 0 \geq L_{l+1}(E) \geq \ldots \geq L_{2 l}(E)>-\infty
$$

and $L_{2 l+1-k}(E)=-L_{k}(E)$ for all $1 \leq k \leq l$.

We are ready to formulate the statement.

Theorem 6.1. Assume that $\omega \in \mathrm{DC}_{t}$. Then all Lyapunov exponents of the operator (6.2) depend continuously on the data, i.e. on $E, \lambda \in \mathbb{R}$ as well as on $W, R, F \in C_{r}^{\omega}\left(\mathbb{T}^{d}, \operatorname{Mat}_{l}(\mathbb{R})\right)$.

Furthermore, given such matrix-valued functions $W, F, R$, there is a constant $\lambda_{0}=\lambda_{0}\left(t, W, F,\|R\|_{r}\right)<\infty$ so that if we fix $\lambda$ with $|\lambda| \geq \lambda_{0}$, the following hold.

(i) (Positivity) If $W$ is not identically singular and if $F$ has no constant eigenvalues, then there is $C_{0}=C_{0}(W, F)<\infty$ such that

$$
L_{l}(E)=L_{l}\left(A_{\lambda, E}\right)>\log |\lambda|-C_{0} \quad \text { for all } E \in \mathbb{R} .
$$

(ii) (Continuity) If $W$ is not identically singular and if $F$ has no constant eigenvalues, then the block $L_{1}+\ldots+L_{l}$ is locally weak-Hölder continuous.

(iii) (Simplicity) Assume that the weight $W$ is one-dimensional, i.e. $W(x)=h(x) I$ where $h \in C_{r}^{\omega}\left(\mathbb{T}^{d}, \mathbb{R}\right)$ with $h(x) \not \equiv 0$. Assume moreover that $F-s I$, seen as an l-dimensional cocycle, has simple Lyapunov exponents for all $s \in \mathcal{E}$, where $\mathcal{E}$ is a compact interval. 
Then the Lyapunov exponents $L_{k}(E)$ of the operator $H_{\lambda}(x)$ are simple for all energies $E \in \lambda \mathcal{E}$. More precisely, there is a constant $\kappa_{0}=\kappa_{0}\left(t, h, F,\|R\|_{r}, \mathcal{E}\right)>0$, such that for all $1 \leq k<l$ and $E \in \lambda \mathcal{E}$,

$$
L_{k}(E)-L_{k+1}(E)>\kappa_{0} .
$$

Proof. The general theorems formulated in Section 1 do not directly apply to the cocycle $A_{\lambda, E}$ above, because it is not defined (and analytic) everywhere. This can be easily remedied by multiplying it with a onedimensional cocycle.

Indeed, by Cramer's formula, whenever $\operatorname{det}[W(x)] \neq 0$, we have $W^{-1}(x)=\frac{1}{\operatorname{det}[W(x)]} \operatorname{adj}(W(x))$.

Let $g(x):=\operatorname{det}[W(x)]$, so $g \in C_{r}^{\omega}\left(\mathbb{T}^{d}, \mathbb{R}\right)$ and $g(x) \neq 0$ a.e. Define

$$
\widetilde{A}_{\lambda, E}(x):=g(x+\omega) A_{\lambda, E}(x)=\left[\begin{array}{cc}
M_{\lambda, E}(x) & N(x) \\
P(x) & Q(x)
\end{array}\right],
$$

where

$$
\begin{aligned}
M_{\lambda, E}(x) & :=U(x)(\lambda F(x)+R(x)-E I) \\
U(x) & :=\operatorname{adj}(W(x+\omega)) \\
N(x) & :=-\operatorname{adj}(W(x+\omega)) W^{T}(x) \\
P(x) & :=g(x+\omega) I \\
Q(x) & \equiv 0 .
\end{aligned}
$$

Then clearly $\widetilde{A}_{\lambda, E} \in C_{r}^{\omega}\left(\mathbb{T}^{d}, \operatorname{Mat}_{m}(\mathbb{R})\right)$ where $m=2 l$, and for all $1 \leq k \leq m$ we have

$$
L_{k}\left(\widetilde{A}_{\lambda, E}\right)=\int_{\mathbb{T}^{d}} \log |g(x)| d x+L_{k}\left(A_{\lambda, E}\right)
$$

By the continuity Theorem 1.2, all Lyapunov exponents are continuous functions on $C_{r}^{\omega}\left(\mathbb{T}^{d}, \operatorname{Mat}_{m}(\mathbb{R})\right)$. The cocycle $\widetilde{A}_{\lambda, E}$ depends continuously on $E, \lambda$ as well as on $W, R, F$ and so the same is true for its Lyapunov exponents. Moreover, the maps $W \mapsto \operatorname{det}[W]$ and $g \mapsto \int_{\mathbb{T}^{d}} \log |g|$ are continuous (the latter may be regarded as the continuity of the Lyapunov exponent of the one-dimensional cocycle $g$ ). By way of formula (6.4), these observations establish the first continuity statement of the theorem.

Now we fix $W, R, F \in C_{r}^{\omega}\left(\mathbb{T}^{d}\right.$, $\left.\operatorname{Mat}_{l}(\mathbb{R})\right)$.

Since $g$ is analytic and $g \not \equiv 0, \int_{\mathbb{T}^{d}} \log |g(x)| d x=C(W)>-\infty$. 
Clearly $\operatorname{det}[U(x)]=\operatorname{det}[\operatorname{adj}(W(x+\omega))]=\operatorname{det}[W(x+\omega)]^{l-1} \not \equiv 0$. Since, moreover, $F$ has no constant eigenvalues, Theorem 1.5 is applicable to $\widetilde{A}_{\lambda, E}$, hence there is $\lambda_{0}$ depending on the fixed data, so that

$$
L_{l}\left(\widetilde{A}_{\lambda, E}\right)>\log |\lambda|-C_{0} \quad \text { for all } E \in \mathbb{R} \text { and for all }|\lambda| \geq\left|\lambda_{0}\right| .
$$

This, together with (6.4), establishes the conclusion of item (i).

Now fix $\lambda$ with $|\lambda| \geq\left|\lambda_{0}\right|$. By item (i), for all energy parameters $E \in \mathbb{R}, L_{l}(E)=L_{l}\left(A_{\lambda, E}\right)>\log |\lambda|-C_{0}>0$ (we may increase $\lambda_{0}$ if necessary). Therefore,

$$
L_{l}(E)>L_{l+1}(E)\left(=-L_{l}(E)\right)
$$

holds for all $E \in \mathbb{R}$

The quantitative statement in Theorem 1.2 is then applicable, and we conclude that the map $E \mapsto\left(L_{1}+\ldots+L_{l}\right)(E)$ is locally weak-Hölder continuous, which establishes item (ii).

We now proceed with the proof of item (iii). Since here we assume that $W(x)=h(x) I$, so $W^{-1}(x)=\frac{1}{h(x)} I$ and $W^{T}(x)=h(x) I$, we can write

$$
\begin{aligned}
h(x+\omega) A_{\lambda, E}(x) & =\left[\begin{array}{cc}
\lambda F(x)+R(x)-E I & -h(x) I \\
h(x+\omega) I & 0
\end{array}\right] \\
& =\lambda\left[\begin{array}{cc}
F(x)+\frac{1}{\lambda} R(x)-\frac{E}{\lambda} I & -\frac{1}{\lambda} h(x) I \\
\frac{1}{\lambda} h(x+\omega) I & 0
\end{array}\right] .
\end{aligned}
$$

Make the change of variables $\delta=\frac{1}{\lambda}, s=\frac{E}{\lambda}$ and define the cocycle

$$
S_{\delta, s}(x):=\left[\begin{array}{cc}
F(x)+\delta R(x)-s I & -\delta h(x) I \\
\delta h(x+\omega) I & 0
\end{array}\right] .
$$

Then clearly $S_{\delta, s} \in C_{r}^{\omega}\left(\mathbb{T}^{d}, \operatorname{Mat}_{m}(\mathbb{R})\right)$ and for all $1 \leq k \leq m$,

$$
L_{k}(E)=L_{k}\left(A_{\lambda, E}\right)=-\int_{\mathbb{T}^{d}} \log |h(x)| d x+\log |\lambda|+L_{k}\left(S_{\frac{1}{\lambda}, \frac{E}{\lambda}} .\right.
$$

Since $h$ is analytic and $h \not \equiv 0$, we have that $\int_{\mathbb{T}^{d}} \log |h(x)| d x \in \mathbb{R}$. Then for all $E \in \mathbb{R}, \lambda \neq 0$ and $1 \leq k<l$,

$$
L_{k}(E)-L_{k+1}(E)=L_{k}\left(S_{\frac{1}{\lambda}, \frac{E}{\lambda}}\right)-L_{k+1}\left(S_{\frac{1}{\lambda}, \frac{E}{\lambda}}\right) .
$$


As $\delta \rightarrow 0$, we have $S_{\delta, s} \rightarrow S_{0, s}=\left[\begin{array}{cc}F-s I & 0 \\ 0 & 0\end{array}\right]$, so by the continuity Theorem 1.2 , if $1 \leq k \leq l$ then

$$
L_{k}\left(S_{\delta, s}\right) \rightarrow L_{k}\left(S_{0, s}\right)=L_{k}(F-s I)
$$

We assumed that for all $s \in \mathcal{E}$, the cocycle $F-s I$ has simple Lyapunov exponents. Then the $l$ largest Lyapunov exponents of $S_{0, s}$ are simple, and so for every fixed $s \in \mathcal{E}$ and every $\delta$ near $0, S_{\delta, s}$ has simple Lyapunov exponents as well.

By the compactness of $\mathcal{E}$ there are $\delta_{0}>0$ and $\kappa_{0}>0$ such that if $(\delta, s) \in\left[-\delta_{0}, \delta_{0}\right] \times \mathcal{E}$ and $1 \leq k<l$, then

$$
L_{k}\left(S_{\delta, s}\right)-L_{k+1}\left(S_{\delta, s}\right)>\kappa_{0} .
$$

Together with 6.5 this proves the claim in item (iii).

Remark 6.1. If for some $s_{0} \in \mathbb{R}$, the cocycle $F-s_{0} I$ has simple Lyapunov exponents, then by the continuity Theorem 1.2 this also holds in a whole neighborhood $\mathcal{E}$ of $s_{0}$. However, this property (and even more so its global counterpart) is difficult to verify, even in the case when $F(x)$ is a diagonal matrix-valued function (unless, of course, the ranges of the diagonal entries are apart from each other). The simplicity statement in the theorem above is therefore not entirely satisfactory.

Next we apply item (ii) - the quantitative continuity result on Lyapunov exponents - in the above theorem to derive a modulus of continuity for the integrated density of states (IDS) of the block Jacobi operators $H_{\lambda}(x)$.

Let us recall a couple of facts.

Denote by $P_{n}$ the coordinate restriction operator to $\{1,2, \ldots, n\} \subset$ $\mathbb{Z}$, and let $H_{\lambda}^{(n)}(x):=P_{n} H_{\lambda}(x) P_{n}^{*}$. It is well know that the following limit exists and it is a.e. independent of $x \in \mathbb{T}^{d}$ :

$$
N(E)=N_{\lambda}(E):=\lim _{n \rightarrow \infty} \frac{1}{n} \#\left((-\infty, E] \cap \text { Spectrum of } H_{\lambda}^{(n)}(x)\right) .
$$

The function $E \mapsto N_{\lambda}(E)$ is called the integrated density of states of the family of ergodic operators $\left\{H_{\lambda}(x): x \in \mathbb{T}^{d}\right\}$.

The IDS is known to be log-Hölder continuous in a very general setting (see [10]). We show that in our context it is in fact weak-Hölder continuous.

It is a classic result in the theory of lattice Schrödinger operators like (1.4) that the Lyapunov exponents and the IDS are related essentially via the Hilbert transform:

$$
L(E)=\int_{\mathbb{R}} \log \left|E-E^{\prime}\right| d N\left(E^{\prime}\right) .
$$


This relation is called the Thouless formula.

Recently, J. Chapman and G. Stolz (see [9]) obtained a Thoulesstype formula (relating the LE and the IDS) which is applicable in the full generality of the operator 6.2 .

Assuming that $C(W):=\int_{\mathbb{T}^{d}} \log |\operatorname{det}[W(x)]| d x>-\infty$, their result states that

$$
\left(L_{1}+\ldots+L_{l}\right)(E)=l \int_{\mathbb{R}} \log \left|E-E^{\prime}\right| d N\left(E^{\prime}\right)-C(W) .
$$

Our assumption on the weight $W(x)$ clearly implies that $C(W)$ is finite. Then by a standard argument involving some properties of the Hilbert transform (see the end of Section 10 in [17]), the relation (6.6) above allows us to transfer the local weak-Hölder modulus of continuity of the map $L_{1}+\ldots+L_{l}$ to the IDS $N(E)$. We then conclude with the following.

Corollary 6.1. Consider the block Jacobi operator (6.2) and assume that the weight $W$ is not identically singular and that the potential function $F$ has no constant eigenvalues. Then its integrated density of states is weak-Hölder continuous, provided $|\lambda|$ is large enough depending on the data.

Remark 6.2. A similar argument also gives a local version of this result. We fix the coupling constant $\lambda$. In other words, let

$$
[H(x) \vec{\psi}]_{n}:=-\left(W_{n+1}(x) \vec{\psi}_{n+1}+W_{n}^{T}(x) \vec{\psi}_{n-1}+R_{n}(x) \vec{\psi}_{n}\right)+F_{n}(x) \vec{\psi}_{n}
$$

be a block Jacobi operator defined by analytic matrix-valued functions and a Diophantine translation vector. If for some energy $E_{0}$ we have that $L_{l}\left(E_{0}\right)>0$, then by the continuity Theorem 1.2 this holds on a small compact neighborhood $\mathcal{E}$ of $E_{0}$. Moreover, by the same continuity theorem the map $\mathcal{E} \ni E \rightarrow\left(L_{1}+\ldots L_{l}\right)(E)$ is weak-Hölder continuous, and so is the IDS $N(E)$.

Acknowledgments. The first author was supported by Fundação para a Ciência e a Tecnologia, under the project: UID/MAT/04561/2013.

The second author was supported by the Norwegian Research Council project no. 213638, "Discrete Models in Mathematical Analysis".

Both authors would like to thank the anonymous referees for their valuable suggestions.

\footnotetext{
${ }^{4}$ Strictly speaking, the result in [9] is formulated under a stronger assumption, but a private conversation with the authors revealed that it holds, in fact, under this more general condition.
} 


\section{REFERENCES}

[1] Artur Ávila, Svetlana Jitomirskaya, and Christian Sadel, Complex onefrequency cocycles, J. Eur. Math. Soc. (JEMS) 16 (2014), no. 9, 1915-1935.

[2] Artur Ávila and Marcelo Viana, Simplicity of Lyapunov spectra: a sufficient criterion, Port. Math. (N.S.) 64 (2007), no. 3, 311-376.

[3] _ Simplicity of Lyapunov spectra: proof of the Zorich-Kontsevich conjecture, Acta Math. 198 (2007), no. 1, 1-56.

[4] C. Bonatti and M. Viana, Lyapunov exponents with multiplicity 1 for deterministic products of matrices, Ergodic Theory Dynam. Systems 24 (2004), no. 5, 1295-1330.

[5] J. Bourgain, Green's function estimates for lattice Schrödinger operators and applications, Annals of Mathematics Studies, vol. 158, Princeton University Press, Princeton, NJ, 2005.

[6] _ Positivity and continuity of the Lyapounov exponent for shifts on $\mathbb{T}^{d}$ with arbitrary frequency vector and real analytic potential, J. Anal. Math. 96 (2005), 313-355.

[7] J. Bourgain and M. Goldstein, On nonperturbative localization with quasiperiodic potential, Ann. of Math. (2) 152 (2000), no. 3, 835-879.

[8] J. Bourgain and S. Jitomirskaya, Continuity of the Lyapunov exponent for quasiperiodic operators with analytic potential, J. Statist. Phys. 108 (2002), no. 5-6, 1203-1218, Dedicated to David Ruelle and Yasha Sinai on the occasion of their 65 th birthdays.

[9] Jacob Chapman and Günter Stolz, Localization for random block operators related to the XY spin chain, Ann. Henri Poincaré 16 (2015), no. 2, 405-435.

[10] Walter Craig and Barry Simon, Log Hölder continuity of the integrated density of states for stochastic Jacobi matrices, Comm. Math. Phys. 90 (1983), no. 2, 207-218.

[11] D Damanik, Schrödinger operators with dynamically defined potentials: a survey, preprint (2015), 1-80, to appear in Ergodic Theory and Dynamical Systems.

[12] Pedro Duarte and Silvius Klein, Positive Lyapunov exponents for higher dimensional quasiperiodic cocycles, Comm. Math. Phys. 332 (2014), no. 1, 189-219.

[13] L Large deviation type estimates for iterates of linear cocycles, Stoch. Dyn. 16 (2016), no. 3, 1660010, 54.

[14] Lyapunov exponents of linear cocycles, Atlantis Studies in Dynamical Systems, vol. 3, Atlantis Press, Paris, 2016, Continuity via large deviations.

[15] Peter L. Duren, Theory of $H^{p}$ spaces, Pure and Applied Mathematics, Vol. 38, Academic Press, New York-London, 1970.

[16] I. Ya. Gol'dsheĭd and G. A. Margulis, Lyapunov exponents of a product of random matrices, Uspekhi Mat. Nauk 44 (1989), no. 5(269), 13-60.

[17] Michael Goldstein and Wilhelm Schlag, Hölder continuity of the integrated density of states for quasi-periodic Schrödinger equations and averages of shifts of subharmonic functions, Ann. of Math. (2) 154 (2001), no. 1, 155-203.

[18] - Fine properties of the integrated density of states and a quantitative separation property of the Dirichlet eigenvalues, Geom. Funct. Anal. 18 (2008), no. $3,755-869$. 
[19] Y. Guivarc'h and A. Raugi, Products of random matrices: convergence theorems, Random matrices and their applications (Brunswick, Maine, 1984), Contemp. Math., vol. 50, Amer. Math. Soc., Providence, RI, 1986, pp. 31-54.

[20] Rui Han and C. A. Marx, Large coupling asymptotics for the lyapunov exponent of quasi-periodic schrödinger operators with analytic potentials, preprint, 2016.

[21] Michael-R. Herman, Une méthode pour minorer les exposants de Lyapounov et quelques exemples montrant le caractère local d'un théorème d'Arnol'd et de Moser sur le tore de dimension 2, Comment. Math. Helv. 58 (1983), no. 3, 453-502.

[22] S. Jitomirskaya and C. A. Marx, Analytic quasi-perodic cocycles with singularities and the Lyapunov exponent of extended Harper's model, Comm. Math. Phys. 316 (2012), no. 1, 237-267.

[23] _ Dynamics and spectral theory of quasi-periodic Schrödinger-type operators, preprint (2015), 1-44, to appear in Ergodic Theory and Dynamical Systems.

[24] Svetlana Jitomirskaya and Rajinder Mavi, Continuity of the measure of the spectrum for quasiperiodic Schrödinger operators with rough potentials, Comm. Math. Phys. 325 (2014), no. 2, 585-601.

[25] Silvius Klein, Localization for quasiperiodic Schrödinger operators with multivariable Gevrey potential functions, J. Spectr. Theory 4 (2014), 1-53.

[26] B. Ya. Levin, Lectures on entire functions, Translations of Mathematical Monographs, vol. 150, American Mathematical Society, Providence, RI, 1996, In collaboration with and with a preface by Yu. Lyubarskii, M. Sodin and V. Tkachenko, Translated from the Russian manuscript by Tkachenko.

[27] Christian Sadel and Disheng Xu, Singular analytic linear cocycles with negative infinite Lyapunov exponents, preprint (2016), 1-16.

[28] Eugene Sorets and Thomas Spencer, Positive Lyapunov exponents for Schrödinger operators with quasi-periodic potentials, Comm. Math. Phys. 142 (1991), no. 3, 543-566.

[29] Zhenghe Zhang, Positive Lyapunov exponents for quasiperiodic Szegö cocycles, Nonlinearity 25 (2012), no. 6, 1771-1797.

Departamento de Matemática and CMafCiO, Faculdade de Ciências, Universidade de Lisboa, Portugal

E-mail address: pmduarte@fc.ul.pt

Departamento de Matemática, Pontifícia Universidade Católica do Rio de Janeiro, Brazil (PUC-Rio)

E-mail address: silviusk@mat.puc-rio.br 